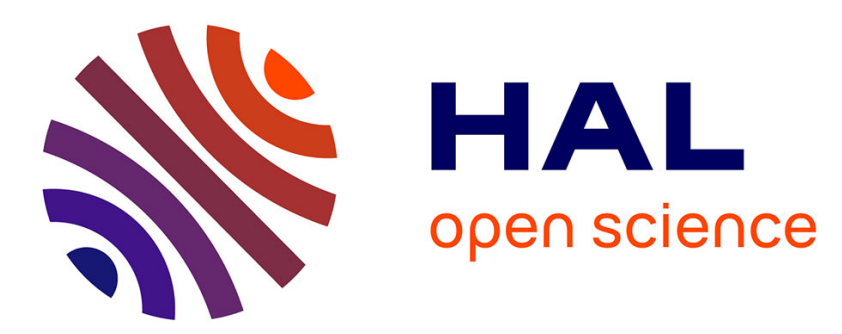

\title{
Linear dynamic analysis of mechanical systems in the medium frequency range
}

\author{
Christian Soize, P.-M. Hutin, A. Desanti, J.-M. David, F. Chabas
}

\section{To cite this version:}

Christian Soize, P.-M. Hutin, A. Desanti, J.-M. David, F. Chabas. Linear dynamic analysis of mechanical systems in the medium frequency range. Computers \& Structures, 1986, 23 (5), pp.605-637. 10.1016/0045-7949(86)90070-2 . hal-00770387

\section{HAL Id: hal-00770387 https://hal.science/hal-00770387}

Submitted on 3 Apr 2013

HAL is a multi-disciplinary open access archive for the deposit and dissemination of scientific research documents, whether they are published or not. The documents may come from teaching and research institutions in France or abroad, or from public or private research centers.
L'archive ouverte pluridisciplinaire HAL, est destinée au dépôt et à la diffusion de documents scientifiques de niveau recherche, publiés ou non, émanant des établissements d'enseignement et de recherche français ou étrangers, des laboratoires publics ou privés. 


\title{
LINEAR DYNAMIC ANALYSIS OF MECHANICAL SYSTEMS IN THE MEDIUM FREQUENCY RANGE
}

\author{
C. Soize, P. M. Hutin, A. Desanti, J. M. David and F. Chabas \\ Office National d'Etudes et de Recherches Aérospatiales 29, Avenue de la Division Leclerc, 92320 \\ Chatillon, France
}

\section{INTRODUCTION}

We will study the dynamic analysis of some general mechanical linear systems in the medium frequency (MF) range. To simplify the mathematical developments, we will only consider the discretized systems which have a finite number of degrees of freedom (DOF). This introduction has four parts. First, we will introduce some notations and definitions. In a second part we will explain the problem in taking a particular and classical mechanical system. In a third part we will briefly introduce the general problem which will be developed in details. In the last part we will give the outline of this paper.

\subsection{Notations and definitions}

Let $\mathbf{U}=\left(\mathbb{U}_{1}, \ldots, \mathbb{U}_{m}\right)$ be a $\mathbb{C}^{m}$ vector and $Q$ a linear operator from $\mathbb{C}^{m}$ to $\mathbb{C}^{n}$. In all this paper, we identify the $\mathbf{U}$ vector with the $(m \times 1)$ column matrix of its $\mathbb{U}_{j}$ components. In the same way, the $Q$ linear operator is identified with its $(n \times m)$ matrix on the canonical basis of $\mathbb{C}^{m}$ and $\mathbb{C}^{n}$. To simplify the notations we take the same symbols for the matrices. The $\mathbb{C}^{m}$ complex vector space is equipped with the usual scalar product and the associated norm:

$$
\begin{gathered}
(\mathbf{U}, \mathbf{V}) \rightarrow\langle\mathbf{U}, \overline{\mathbf{V}}\rangle_{m}=\Sigma_{j=1}^{m} \mathbb{U}_{j} \overline{\mathbb{V}}_{j}, \\
\mathbf{U} \rightarrow\|\mathbf{U}\|_{m}=\langle\mathbf{U}, \overline{\mathbf{U}}\rangle_{m}^{1 / 2},
\end{gathered}
$$

where $\bar{V}_{j}$ is the complex conjuguate.

Let $H_{m}=L^{2}\left(\mathbb{R}, \mathbb{C}^{m}\right)$ be the Hilbert vector space of all the $\mathbb{C}^{m}$-valued mappings $t \rightarrow \mathrm{U}(t)$ almost everywhere (a.e.) defined on $\mathbb{R}$ such that

$$
\|\mathbf{U}\|_{m}=\left(\int_{R}\|\mathbf{U}(t)\|_{m}^{2} \mathrm{~d} t\right)^{1 / 2}<+\infty .
$$

The mapping $\mathbf{U} \rightarrow\|\| \mathbf{U}\|\|_{m}$ from $H_{m}$ to $\mathbb{R}^{+}$is a norm on $H_{m}$. The associated scalar product is written as

$$
(\mathbf{U}, \mathbf{V}) \rightarrow\langle\langle\mathbf{U}, \overline{\mathbf{V}})\rangle_{m}=\int_{\mathbb{R}}\langle\mathbf{U}(t), \overline{\mathbf{V}(t)}\rangle_{m} \mathrm{~d} t .
$$

For any $\mathbf{V} \in H_{m}$, the Fourier transform (FT) of $\mathbf{V}$ is the mapping $\omega \rightarrow(\mathscr{F} \mathbf{V})(\omega)=\hat{\mathbf{V}}(\omega)$ belonging to $H_{m}$, such that for a.e. $\omega \in \mathbb{R}$ :

$$
\hat{V}_{j}(\omega)=\int_{\mathbb{R}} \mathrm{e}^{-i \omega t} V_{j}(t) \mathrm{d} t, \quad j \in\{1, \ldots, m\} .
$$

For a.e. $t \in \mathbb{R}$, the inverse $\mathrm{FT}$ is given by

$$
V_{j}(t)=\frac{1}{2 \pi} \int_{\mathbb{R}} \mathrm{e}^{i \omega t} \hat{V}_{j}(\omega) \mathrm{d} \omega, \quad j \in\{1, \ldots, m\} .
$$

We have the Plancherel's equality for any $\mathbf{U}$ and $\mathbf{V}$ in $H_{m}$ :

$$
\begin{aligned}
\langle\langle\mathbf{U}, \overline{\mathbf{V}}\rangle\rangle_{m} & =\frac{1}{2 \pi}\langle\langle\hat{\mathbf{U}}, \overline{\hat{\mathbf{V}}}\rangle\rangle_{m} ; \\
\|\| \mathbf{U}\|\|_{m} & =\frac{1}{\sqrt{2 \pi}}\|\hat{\mathbf{U}}\| \|_{m} .
\end{aligned}
$$

\subsection{Particular case of an elastic structure in vacuo}

Let us consider a linear elastic structure in vacuo, i.e. without external or internal fluid and so on. Theoretically, the linear vibrations of an elastic, viscous, heterogeneous, anisotropic structure occupying a bounded domain in $\mathbb{R}^{3}$ space, slightly damped, can be studied without difficulty if we know explicitly the spectrum $\left\{\omega_{j}\right\}, j \in \mathbb{N}$ of eigenfrequencies for the associated undamped system, and the corresponding modal basis $\left\{\phi_{j}\right\}, j \in \mathbb{N}$. In practice, for such a structure, the modal basis is not explicitly known and must be calculated numerically by using finite element method.

For the high frequency range, the excitation frequencies are high enough to use specific methods, like that, asymptotic methods and the SEA method.

For the low frequency (LF) range, the excitation frequencies are low enough for the response to be of the modal type. Therefore, only the first modes of the structure intervene in the response.

We are interested in the MF range which is the intermediary frequency range. In this MF range, all the principal elements of the structure, the "exact" geometry, the boundary conditions play a fundamental part in the response of the mechanical system. Let us note first of all that the structure must be finely discretized into finite elements in this MF range. This leads us to reason using discretized sys- 
tems with a large number of DOF. For instance, 30 000 or 40000 DOF's may be necessary.

Let $m$ be the number of DOF. Let $\mathbf{M}_{\mathbf{S}}, \mathbf{C}_{\mathbf{S}}$, and $\mathbf{K}_{\mathbf{S}}$ be, respectively, the mass, damping, and stiffness $(m \times m)$ real, symmetric, and positive definite matrices of the discretized linear heterogeneous anisotropic viscous elastic structure. The vibrations of this structure are fully described by the frequency response function (f.r.f.) which is the continuous and bounded mapping $\omega \rightarrow \mathbf{T}(\omega)$ defined on $\mathbb{R}$ with values in the $(m \times m)$ complex symmetric matrices:

$$
\mathbf{T}(\omega)=\left[-\omega^{2} \mathbf{M}_{\mathbf{S}}+i \omega \mathbf{C}_{\mathbf{s}}+\mathbf{K}_{\mathbf{s}}\right]^{-1} .
$$

The $T(\omega)$ matrix is associated to the linear differential equation on $\mathbb{C}^{m}$, which governs the linear vibrations of the discretized system

$$
\mathbf{M}_{\mathbf{S}} \ddot{\mathbf{U}}(t)+\mathbf{C}_{\mathbf{S}} \dot{\mathbf{U}}(t)+\mathbf{K}_{\mathbf{S}} \mathbf{U}(t)=\mathbf{F}(t),
$$

where $\mathbf{U}(t)$ is the $(m \times 1)$ column matrix of the unknown nodal displacements. $\dot{\mathbf{U}}(t)=\mathrm{d} \mathbf{U}(t) / \mathrm{d} t$, $\ddot{\mathrm{U}}(\mathrm{t})=\mathrm{d}^{2} \mathrm{U}(t) / \mathrm{d} t^{2}$, respectively, the velocity and the acceleration. The $(m \times 1)$ column matrix $\mathbf{F}(t)$ represents the equivalent nodal excitation forces. At the present time we consider deterministic excitation.

Let be $\mathbf{F} \in H_{m}$; then $\hat{\mathbf{F}} \in H_{m}$. Let $\hat{\mathbf{U}}$ be the FT of $\mathbf{U}$ defined by the following equation for a.e. $\omega$ in $\mathbb{R}$ :

$$
\hat{\mathbf{U}}(\omega)=\mathbf{T}(\omega) \hat{\mathbf{F}}(\omega) .
$$

Then $\hat{\mathbf{U}}$ is into $H_{m}$ and $\hat{\mathbf{U}}$ is usually called the forced vibrations. Indeed, if $\tilde{U}(t)$ is the solution of eqn (9) for $t>t_{0}$, such that $\tilde{\mathbf{U}}\left(t_{0}\right)=\mathbf{U}_{0}$ and $\dot{\mathbf{U}}\left(t_{0}\right)=\mathbf{U}_{1}$, then

$$
\lim _{t \rightarrow+\infty}\|\mathbf{U}(t)-\tilde{\mathbf{U}}(t)\|_{m}=0 .
$$

Let us investigate what are the possibilities to apply LF method for solving MF problem. In LF range, linear vibrations of structures can classically be solved: $M 1-B y$ direct numerical time integration of eqn (9); $M 2$ - by a numerical time integration of the equations in the truncated modal basis $\left\{\Phi_{1}, \ldots\right.$, $\left.\Phi_{p}\right\}$ with $p \ll m$. This is the modal synthesis in the time domain. M3-By calculating the f.r.f T $(\omega)$ defined by eqn ( 8 ), which can be (a) carried out using the truncated modal basis:

$$
\mathbf{T}(\omega) \sim \sum_{j, l=1}^{p} h_{j l}(\omega) \Phi_{j} \Phi_{l}^{T},
$$

where $h_{j l}(\omega)$ is the f.r.f. relating to the modal coordinates. This is the modal synthesis in the frequency domain; (b) or carried out directly calculating, for each value of $\omega$ considered, the $T(\omega)$ matrix given by eqn (8).

If applied to MF, the methods described above for LF would lead to the following adaptations in use:

(1) For a broadband MF excitation, the $M 1$ method requires a long integration time with a very small integration time step. Consequently, the total number of the integration time steps is large. As the order of the matrices is very large (e.g. $m=30000$ ), there are many numerical difficulties (numerical noises. . .). On the other hand, the data processing of a such job is tricky. Nevertheless, the substructuring technique is easy.

(2) Methods $M 2$ and $M 3$ (a) require the calculation of the vibration eigenmodes up to a high order (e.g. 1000 eigenmodes). As the discretized system has a large number of DOF, it seems difficult (1) to directly determine the necessary truncated modal basis and (2) to compute with enough precision the eigenmodes and to separate numerically the eigenvalues, considering the current state of the art in numerical analysis for solving a large generalized eigenvalue problem. The substructuring analysis is possible, but not very easy. At the present time and for the MF problem, the modal approach is not really efficient and is very expensive in CPU time and $\mathrm{I} / \mathrm{O}$. Method $M 3$ (b) involves finding the solution to the complex linear system:

$$
\left[-\omega^{2} \mathbf{M}_{\mathbf{S}}+i \omega \mathbf{C}_{\mathbf{S}}+\mathbf{K}_{\mathbf{S}}\right] \hat{\mathbf{U}}(\omega)=\hat{\mathbf{F}}(\omega),
$$

carried out for each $\omega$. If the calculation is to be carried out only for a few values of $\omega$, this method is then very effective in the MF range. This is true when the excitation is one or several rays. But if the excitation is a broadband signal, as is often the case for both deterministic and stationary random excitations in the MF domain, numerous values of $\omega$ must be considered. It is also the case for a direct broadband dynamic identification (e.g. 2000-4000 $\mathrm{Hz}$ ). Moreover, method $M 3$ (b) should normally lead to the consideration of very many values of $\omega$, because the elastic medium being bounded and slightly damped a priori, it seems difficult to assume a slow variation of the mapping $\omega \rightarrow \mathbf{T}(\omega)$ in an MF band before any calculations are made. This excludes, a priori, a calculation involving only a few values of $\omega$ and the cost of this method may then appear prohibitive. We present in this paper a numerical method for the MF range, based on the following principles. An MF broadband $B \subset \mathbb{P}^{+}$is written as $B=\cup_{n} B_{n}$, where $B_{n}$ is a narrow MF band. For each $B_{n}$ band, the vibration equation will be directly solved by a technique of multiple scales which uses, simultaneously the advantages of both the $M 1$ and $M 3$ (b) methods. Moreover, the substructuring technique is very easy to use. On the other hand, the proposed MF method allows one to solve the general case which is introduced in the next section.

\subsection{The general problem considered}

In the last section, the $\mathbf{M}_{\mathbf{S}}, \mathbf{C}_{\mathbf{S}}$, and $\mathbf{K}_{\mathbf{S}}$ matrices do not depend on $\omega$. In the present paper we are 
considering the MF vibrations of the discretized system governed in the Fourier domain by the following linear equation on $\mathbb{C}^{m}$ :

$$
\left[-\omega^{2} \mathbf{M}(\omega)+i \omega \mathbf{C}(\omega)+\mathbf{K}(\omega)\right] \hat{\mathbf{U}}(\omega)=\hat{\mathbf{F}}(\omega),
$$

where the $\mathbf{M}(\omega), \mathbf{C}(\omega)$, and $\mathbf{K}(\omega)$ matrices depend on $\omega \in \mathbb{R}$. For instance we have this equation for an elastic structure with viscoelastic materials, or for an hydroelasticity problem, i.e. for an elastic body coupled with an unviscous compressible unbounded external fluid.

On the other hand we shall consider some deterministic and random excitations.

\subsection{Outline of this paper}

This paper contains two parts: (a) In part I, we will present a method for studying the vibrations of general linear mechanical systems in the MF domain. We will develop the theory of the proposed MF method. We will establish the formulas which allow to compute directly the response of the mechanical system. At the end of this first part, we will speak about the implantation of this method in the ADINA program[3] on scalar and vectorial computers.

(b) Part II concerns the applications. In a first section we will study the linear MF vibrations of elastic structures in vacuo. The second section concerns the hydroelasticity problems. We will study the MF vibrations of an elastic structure coupled with a perfect compressible unbounded external dense fluid. In the third section we will study the elastic structures coupled with a viscous compressible bounded internal fluid. The fourth section concerns an elastic structure with viscoelastic material.

We will give, for each of these cases: (1) some theoretical elements, (2) the corresponding development in ADINA, (3) some comments on the validation, and (4) some concrete examples, with experimental comparisons in several cases.

\section{PART I: THEORY OF THE PROPOSED MF METHOD}

\subsection{Vibration equation}

We consider the MF vibrations of a discretized system which is governed in the Fourier space by eqn (14) on $\mathbb{C}^{m}$ with the following hypotheses: (1) The functions $\omega \rightarrow \mathbf{M}(\omega), \omega \rightarrow \mathbf{C}(\omega)$ and $\omega \rightarrow \mathbf{K}(\omega)$ with values in the $(m \times m)$ real symmetric matrices are continuous on $\mathbb{R}$. (2) For $Q(\omega) \in\{\mathbf{M}(\omega), \mathbf{C}(\omega)$, $\mathbf{K}(\omega)\}$, there is a finite real positive constant $k_{Q}$ such that $\forall V \in \mathbb{R}^{m}$, $\inf _{\omega \in \mathbb{R}}\langle Q(\omega) V, V\rangle_{m} \geqslant k_{Q}\|V\|_{m}^{2}$. Then $\forall \omega \in \mathbb{R}, \mathbf{M}(\omega), \mathbf{C}(\omega)$, and $\mathbf{K}(\omega)$ are positive definite matrices. Consequently, the frequency response function:

$$
\omega \rightarrow \mathbf{T}(\omega)=\left[-\omega^{2} \mathbf{M}(\omega)+i \omega \mathbf{C}(\omega)+\mathbf{K}(\omega)\right]^{-1}
$$

is a continuous and bounded mapping on $\mathbb{R}$ with values in the $(m, \times, m)$ complex matrices [but $\mathbf{T}(\omega)$ is not Hermitian].
For any $\mathbf{F} \in H_{m}$, the solution of eqn (14) is unique and $\omega \rightarrow \hat{\mathbf{U}}(\omega)=\mathbf{T}(\omega) \hat{\mathbf{F}}(\omega)$ is such that $\mathbf{U} \in$ $H_{m}$.

\subsection{Narrow band signal (NBS). The set $H_{m}\left(B_{n}\right)$}

Let $B_{n}=\left[\Omega_{n}-\Delta \omega / 2, \Omega_{n}+\Delta \omega / 2\right]$ be a compact frequency band of $\mathbb{R}^{+}$with a central frequency $\Omega_{n}$ $>0$ and a bandwidth $\Delta \omega$ such that $0<\Delta \omega<2 \Omega_{n}$. We denote by $B_{0}$ the band $[-\Delta \omega / 2, \Delta \omega / 2]$. Let $\tau_{\mathrm{L}}$ and $\tau_{\mathrm{c}}$ be such that

$$
\tau_{\mathrm{L}}=\frac{2 \pi}{\Delta \omega} ; \quad \tau_{\mathrm{c}}=\frac{2 \pi}{\Omega_{n}} .
$$

Let $H_{m}\left(B_{n}\right)$ be the set of all $\mathbb{C}^{m}$-valued function $t$ $\rightarrow \mathbf{F}_{n}(t)$ a.e. defined on $\mathbb{R}$ such that

$$
H_{m}\left(B_{n}\right)=\left\{\mathbf{F}_{n} \in H_{m} ; \hat{\mathbf{F}}_{n}(\omega)=0, \forall \omega \notin B_{n}\right\} .
$$

Let us note that $H_{m}=H_{m}(\overline{\mathbb{R}})$.

A signal $\mathbf{F}_{n}$ is called a narrow band signal (NBS) (1) if $\mathbf{F}_{n} \in H_{m}\left(B_{n}\right)$ and (2) if $\tau_{\mathrm{c}} / \tau_{\mathrm{L}}=\Delta \omega / \Omega_{n} \ll 1$. The small time scale is $\tau_{\mathrm{c}}$ and the large time scale is $\tau_{\mathrm{L}}$.

If $\mathbf{F}_{n}$ is a NBS, we can associate with $\mathbf{F}_{n}$ a low frequency signal (LFS) $\mathbf{F}_{0}$ belonging to $H_{m}\left(B_{0}\right)$, such that for a.e. $t$ in $\mathbb{R}$ and $\omega^{\prime}$ in $B_{0}$ :

$$
\begin{aligned}
\mathbf{F}_{0}(t) & =\mathbf{F}_{n}(t) \mathrm{e}^{-i \Omega_{n} t} \\
\hat{\mathbf{F}}_{0}\left(\omega^{\prime}\right) & =\hat{\mathbf{F}}_{n}\left(\omega^{\prime}+\Omega_{n}\right) .
\end{aligned}
$$

The LFS $F_{0}$ has one an only one time scale, the $\tau_{\mathbf{L}}$ scale.

\subsection{Examples of excitation and associated responses}

2.3.1 Narrow band deterministic excitation.

(a) Let $\mathbf{F}_{n}$ be a NBS on $B_{n}$. Then denoting by $\mathrm{U}_{n}$ the solution, we have for a.e. $\omega \in B_{n}$ :

$$
\hat{\mathbf{U}}_{n}(\omega)=\mathbf{T}(\omega) \hat{\mathbf{F}}_{n}(\omega)
$$

and we see that $\mathbf{U}_{n}, \dot{\mathbf{U}}_{n}$, and $\ddot{\mathbf{U}}_{n} \in H_{m}\left(B_{n}\right)$ are NBSs.

(b) Let us study a particular case. Let $\omega \rightarrow$ $\hat{\mathbf{1}}_{B_{n}}(\omega)$ be the $\mathbb{R}$-valued function defined on $\mathbb{R}$ such that $\hat{\mathbf{1}}_{B_{n}}(\omega)=0$ if $\omega \notin B_{n}$ and $\hat{\mathbf{1}}_{B_{n}}(\omega)=1$ if $\omega \in B_{n}$. For any $j \in\{1, \ldots, m\}$, let $\mathbf{F}_{n, j}$ be a NBS on $B_{n}$ such that $\hat{\mathbf{F}}_{n, j}(\omega)=\hat{\mathbf{1}}_{B_{n}}(\omega) \mathbf{A}_{j}$, where $\mathbf{A}_{j}$ is the $(m \times$ 1) complex column matrix such that $\left[\mathbf{A}_{j}\right]_{k}=\delta_{j k} \cdot\left(\delta_{j k}\right.$ $=0$ if $\left.j \neq k, \delta_{j j}=1\right)$. Let $\hat{\mathbf{U}}_{n, j}(\omega)=\mathbf{T}(\omega) \hat{\mathbf{F}}_{n, j}(\omega)$ be the response. Thus, we see that for $\omega \in B_{n}$, all the elements $[\mathbf{T}(\omega)]_{k j}=\left[\hat{\mathbf{U}}_{n, j}(\omega)\right]_{k}$, for $k \in\{1, \ldots, m\}$, of the f.r.f. $\omega \rightarrow \mathbf{T}(\omega)$ are obtained by calculating the response of the system to the particular excitation $\mathbf{F}_{n, j}$. Let us note that the LFS: $\left[t \rightarrow \mathbf{1}_{B_{0}}(t)\right]$ $\in H_{1}\left(B_{0}\right)$ associated with the NBS: $\left[t \rightarrow \mathbf{1}_{B_{n}}(t)\right] \in$ $H_{1}\left(B_{n}\right)$ is written as $\mathbf{1}_{B_{0}}(t)=(\pi t)^{-1} \sin \left(\pi t / \tau_{\mathbf{L}}\right)$.

2.3.2 Broadband deterministic excitation.

Let $B=\left[\omega_{\mathbf{I}}, \omega_{\mathrm{S}}\right] \subset \mathbb{R}^{+}$be a compact broad MF band. The given deterministic excitation $\mathbf{F}$ is an element of $H_{m}(B)$. Let $N$ be a finite positive integer. 
We write $B=\cup_{n=1}^{N} B_{n}, \cap_{n=1}^{N} B_{n}=\varnothing$, where each $B_{n}$ is a narrow MF band. Then, we have

$$
\hat{\mathbf{F}}(\omega)=\sum_{n=1}^{N} \hat{\mathbf{1}}_{B_{n}}(\omega) \hat{\mathbf{F}}_{n}(\omega),
$$

where $\mathbf{F}_{n} \in H_{m}\left(B_{n}\right)$ is a NBS on $B_{n}$. Then using eqn (19), we see that the solution $\hat{\mathbf{U}}$ of eqn (14) is written as

$$
\hat{\mathbf{U}}(\omega)=\sum_{n=1}^{N} \hat{\mathbf{1}}_{B_{n}}(\omega) \hat{\mathbf{U}}_{n}(\omega),
$$

where $\mathbf{U}_{n}, \dot{\mathbf{U}}_{n}, \ddot{\mathbf{U}}_{n} \in H_{m}\left(B_{n}\right)$ are NBSs on $B_{n}$, and $\mathbf{U}, \dot{\mathbf{U}}, \ddot{\mathbf{U}} \in H_{m}(B)$. Using 2.3.1 (b) we can identify $\mathbf{T}(\omega)$ on the broad band $B$.

2.3.3 Narrow band stationary random excitation.

2.3.3(a) The set $H S_{m}\left(B_{n}\right)$. Let $B_{n}$ be a narrow MF band and $\mathbf{B}_{n}=\left[-\left(\Omega_{n}+\Delta \omega / 2\right),-\left(\Omega_{n}-\Delta \omega /\right.\right.$ 2)]. Let $\{\mathbf{X}(t), t \in \mathbb{R}\}$ be an $\mathbb{R}^{m}$-valued, second order, centered, stationary, and continuous in quadratic mean stochastic process. Let $\tau \rightarrow R_{\mathbf{X}}(\tau)$ $=\mathscr{E}\left[\mathbf{X}(t+\tau) \mathbf{X}(t)^{T}\right]$ be the autocorrelation function continuous on $\mathbb{R}$, with values in the $(m \times m)$ real symmetric matrices ( $\mathscr{E}$ designates the mathematical expectation). We suppose:

(1) that the spectral measure has a density $S_{\mathbf{X}}$ :

$$
R_{\mathbf{X}}(\tau)=\int_{\mathbb{R}} \mathrm{e}^{i \omega \tau} S_{\mathbf{X}}(\omega) \mathrm{d} \omega
$$

The spectral density function (s.d.f.) $\omega \rightarrow S_{\mathbf{X}}(\omega)$ is defined on $\mathbb{R}$ with values in the $(m \times m)$ complex Hermitian matrices $\left[S_{\mathbf{X}}(\omega)=S_{\mathbf{X}}(\omega)^{*}\right]$;

(2) that the s.d.f. has a compact support $B_{n} \cup$ $\mathbf{B}_{n}$, i.e. $\forall \omega \notin B_{n} \cup \mathbf{B}_{n}, S_{\mathbf{X}}(\omega)=0$. Let $\mathbf{C}_{\mathbf{X}}$ be the $(m \times m)$ real covariance matrix such that

$$
\mathbf{C}_{\mathbf{X}}=R_{\mathbf{X}}(0)=2 \int_{B_{n}} \operatorname{Re} S_{\mathbf{X}}(\omega) \mathrm{d} \omega,
$$

where $\mathrm{Re}$ designates the real part. Then we know that that

(1) the total power $\mathscr{P}_{\mathbf{X}}$ of the process $\mathbf{X}(t)$ is such

$$
\mathscr{P}_{\mathbf{X}}=\operatorname{Tr} \mathbf{C}_{\mathbf{X}}=\Sigma_{j=1}^{m}\left[\mathbf{C}_{\mathbf{X}}\right]_{j j}=\mathscr{E}\left(\|\mathbf{X}(t)\|_{m}^{2}\right)<+\infty .
$$

(2) $\mathscr{P}_{\mathbf{X}}>0$, because we exclude the trivial case where $\mathbf{X}$ is the null constant vector.

(3) $\mathbf{C}_{\mathbf{X}}$ is a $(m \times m)$ real symmetric positive definite matrix.

Such a process is called a narrow band stationary random process. The set of all the stochastic processes which verify all the preceding properties is denoted by $H S_{m}\left(B_{n}\right)$.

2.3.3(b) Stochastic response for an excitation in $H S_{m}\left(B_{n}\right)$. Let us consider the $(m \times m)$ convolution linear filter whose f.r.f. $\omega \rightarrow \mathbf{T}(\omega)$ is defined by eqn (15). If the input of this linear filter is a stochastic process $\mathbf{F}_{n}$ belonging to $H S_{m}\left(B_{n}\right)$, consequently we know $[29,44,60]$ that (1) the output process denoted by $\mathbf{U}_{n}$ belongs to $H S_{m}\left(B_{n}\right)$, (2) the quadratic mean derivatives $\dot{\mathbf{U}}_{n}(t), \quad \ddot{\mathbf{U}}_{n}(t)$ exist and belong to $H S_{m}\left(B_{n}\right)$, and (3) the s.d.f. $S_{\mathbf{U}_{n}}(\omega)$ of $\mathbf{U}_{n}$ is such that $S_{\mathrm{U}_{n}}(\omega)=\mathbf{T}(\omega) S_{\mathbf{F}_{n}}(\omega) \mathbf{T}(\omega)^{*}$.

2.3.3(c) Particular case. Let be $J \in\{1, \ldots, m\}$ and $\Psi_{1}, \ldots, \Psi_{J}, J$ given vectors of $\mathbb{R}^{m}$. Let $\mathbf{F}_{n} \in$ $H S_{m}\left(B_{n}\right)$ the random excitation defined by

$$
\mathbf{F}_{n}(t)=\Sigma_{j=1}^{J} \mathbf{X}_{j}(t) \Psi_{j},
$$

where $\mathbf{X}=\left(\mathbf{X}_{1}, \ldots, \mathbf{X}_{J}\right)$ is a given stochastic process belonging to $H S_{J}\left(B_{n}\right)$. Under these conditions we can easily prove that

$\forall \omega \in B_{n}$,

$$
\begin{aligned}
S_{\mathbf{U}_{n}}(\omega) & =\sum_{j, k=1}^{J}\left[S_{\mathbf{X}}(\omega)\right]_{j k} \hat{\mathbf{U}}_{n, j}(\omega) \hat{\mathbf{U}}_{n, k}(\omega)^{*}, \\
\forall \omega & \in \mathbf{B}_{n}, \\
S_{\mathbf{U}_{n}}(\omega) & =\overline{S_{\mathbf{U}_{n}}\left(\omega^{\prime}\right)}, \\
\omega^{\prime} & =-\omega \in B_{n},
\end{aligned}
$$

where, for $j \in\{1, \ldots, J\}, \mathbf{U}_{n, j} \in H_{m}\left(B_{n}\right)$ is the NBS on $B_{n}$ such that

$$
\hat{\mathbf{U}}_{n, j}(\omega)=\mathbf{T}(\omega) \hat{\mathbf{F}}_{n, j}(\omega),
$$

and where $\mathbf{F}_{n, j} \in H_{m}\left(B_{n}\right)$ is the NBS on $B_{n}$ such that $\hat{\mathbf{F}}_{n, j}(\omega)=\hat{\mathbf{1}}_{B_{n}}(\omega) \Psi_{j}$. The relations (25) and (26) show that the second order quantities of $\mathbf{U}_{n}$ can be obtained by calculating $J$ deterministic responses $\mathbf{U}_{n, j}$ due to $J$ narrow band deterministic excitations $\mathbf{F}_{n, j}$.

2.3.3(d) General case. Let $\mathbf{F}_{n}$ be in $H S_{m}\left(B_{n}\right)$. Let $\lambda_{j}>0$ and $\Psi_{j} \in \mathbb{R}^{m}$ be, respectively, an eigenvalue and an eigenvector of the covariance matrix $\mathrm{C}_{\mathrm{F}}$ such that

$$
\mathbf{C}_{\mathbf{F}_{n}} \Psi_{j}=\lambda_{j} \Psi_{j}
$$

We know that there is a normalization such that the set of eigenvectors $\left\{\Psi_{1}, \ldots, \Psi_{m}\right\}$ is an orthonormal basis of $\mathbb{R}^{m}:\left\langle\Psi_{j}, \Psi_{k}\right\rangle_{m}=\delta_{j k}$. We order the $\lambda_{j} \mathrm{~s}$ in decreasing values: $\lambda_{1} \geqslant \lambda_{2} \geqslant \cdots \geqslant \lambda_{m}>0$. Thus, (1) the process $\mathbf{F}_{n}$ is written as $\mathbf{F}_{n}(t)=\sum_{j=1}^{m} \mathbf{X}_{j}(t) \Psi_{j}$, where $\mathbf{X}=\left(\mathbf{X}_{1}, \ldots, \mathbf{X}_{m}\right)$ belongs to $H S_{m}\left(B_{n}\right)$ and has an s.d.f. $S_{\mathbf{X}}(\omega)$ given by $\left[S_{\mathbf{X}}\right]_{j k}=\left\langle S_{\mathbf{F}_{n}}(\omega) \Psi_{k}\right.$, $\left.\Psi_{j}\right\rangle_{m} ;(2)$ the total power $\mathscr{P}_{\mathbf{F}_{n}}$ of the process $\mathbf{F}_{n}$ is $\sum_{j=1}^{m} \lambda_{j} ;(3)$ for any $\epsilon>0$, there is a positive integer $J_{\epsilon} \leqslant m$ such that $\mathscr{P}_{\mathbf{F}_{n}}-\sum_{j=1}^{J_{\epsilon}} \lambda_{j} \leqslant \epsilon$.

This projection is of interest if $m$ is very large (which is our case), and if for a small enough fixed $\epsilon$, we have $J_{\epsilon} \ll m$. In this case we can write

$$
\mathbf{F}_{n}(t) \sim \sum_{j=1}^{J_{\epsilon}} \mathbf{X}_{j}(t) \Psi_{j}
$$

The approximation (28) is taken in the quadratic mean sense. The random excitation (28) is of the type (24) and the results of the particular case are 
applicable (e.g. $m=30000$ and $J_{\mathbf{E}}=10$ ). Let us note that generally the excitation is not applied on all the DOFs, but only on $m^{\prime}$ DOFs of the system with $m^{\prime} \ll m$ (e.g. $\left.m^{\prime}=2000\right)$. In this case, the eigenvalue problem (27) must be solved on the condensed equation to determine the $J_{\epsilon}$ first eigenvalues and eigenvectors.

2.3.4 Broadband stationary random excitation. Let $\mathbf{F}$ be a random excitation belonging to $H S_{m}(B)$, where $B$ is the compact broad MF band of Sec. 2.3.2. Using the same method, we can write $S_{\mathrm{F}}(\omega)$ $=\sum_{n=1}^{N} \hat{\mathbf{1}}_{B_{n}}(\omega) S_{\mathbf{F}_{n}}(\omega)$ with $\mathbf{F}_{n} \in H S_{m}\left(B_{n}\right)$. Then, we have $S_{\mathrm{U}}(\omega)=\sum_{n=1}^{N} \hat{\mathbf{1}}_{B_{n}}(\omega) S_{\mathrm{U}_{n}}(\omega)$ and $S_{\mathrm{U}_{n}}(\omega)$ is calculated with eqn (25).

\subsection{Model equation for the MF vibrations}

The results of Sec. 2.3 show that for a large class of deterministic and random excitations, the solution is obtained by solving only some problems with a narrow band deterministic excitation (see Sec. 2.3.1). Then, we must find the NBS $\mathrm{U}_{n} \in H_{m}\left(B_{n}\right)$ such that, for a given NBS, $\mathbf{F}_{n} \in H_{m}\left(B_{n}\right)$, we have for a.e. $\omega$ in $B_{n}$ :

$$
\left[-\omega^{2} \mathbf{M}(\omega)+i \omega \mathbf{C}(\omega)+\mathbf{K}(\omega)\right] \hat{\mathbf{U}}_{n}(\omega)=\hat{\mathbf{F}}_{n}(\omega) .
$$

Let us introduce the auxiliary equation to eqn (29). Let $\mathbf{U}_{n} \in H_{m}\left(B_{n}\right)$ be the NBS such that for a.e. $\omega$ in $B_{n}$ :

$$
\left[-\omega^{2} \mathbf{M}_{n}+i \omega \mathbf{C}_{n}+\mathbf{K}_{n}\right] \hat{\mathbf{U}}_{n}(\omega)=\hat{\mathbf{F}}_{n}(\omega),
$$

where $\mathbf{F}_{n}$ is the same NBS on $B_{n}$ as in eqn (29), and where

$$
\mathbf{M}_{n}=\mathbf{M}\left(\Omega_{n}\right), \quad \mathbf{C}_{n}=\mathbf{C}\left(\Omega_{n}\right), \quad \mathbf{K}_{n}=\mathbf{K}\left(\Omega_{n}\right) .
$$

we let

$$
\mathbf{T}_{n}(\omega)=\left[-\omega^{2} \mathbf{M}_{n}+i \omega \mathbf{C}_{n}+\mathbf{K}_{n}\right]^{-1} .
$$

Taking into account the results of Sec. 2.1, we conclude that the functions $\omega \rightarrow \mathrm{T}(\omega)$ and $\omega \rightarrow \mathrm{T}_{n}(\omega)$ are continuous on the compact interval $B_{n}$. Thus, for any fixed $\epsilon>0$, there is $\delta \omega(\epsilon) \in] 0, \Delta \omega]$ such that

$$
\int_{\Omega_{n}-\delta \omega(\epsilon) / 2}^{\Omega_{n}+\delta \omega(\epsilon) / 2}\left\|\hat{\mathbf{U}}_{n}(\omega)-\hat{\mathbf{U}}_{n}(\omega)\right\|_{m}^{2} \mathrm{~d} \omega \leqslant \epsilon .
$$

The inequality (33) shows that one can always take a band $B_{n}$ with a small enough bandwidth $\Delta \omega$ such that $\underline{\mathbf{U}}_{n} \sim \mathbf{U}_{n}$. For the numerical point of view, this approximation is interesting if the continuous function $\omega \rightarrow \mathbf{M}(\omega), \mathbf{C}(\omega)$, and $\mathbf{K}(\omega)$ are smooth functions. Thus, for a fixed $\epsilon$, the bandwidth $\Delta \omega$ is not too small and the construction of the solution on a broadband $B=\cup_{n=1}^{N} B_{n}$ does not require a large value of $N$.

2.4.1 Model equation in the Fourier space. Equation (31) is. called the model equation in the
Fourier space for the MF vibrations. The corresponding MF model problem can be formulated as follows: Find the NBS $\mathrm{U}_{n} \in H_{m}\left(B_{n}\right)$ which verifies eqn (30) for a given NBS $\mathbf{F}_{n}$ in $H_{m}\left(B_{n}\right)$.

This solution is written as $\hat{\mathbf{U}}_{n}(\omega)=\mathrm{T}_{n}(\omega)$ $\hat{\mathbf{F}}_{n}(\omega)$ for a.e. $\omega$ in $B_{n}$, but obviously, we will build this solution without directly computing the matrix $\mathbf{T}_{n}(\omega)$, i.e. without solving the complex linear system (30) for many values of $\omega$ in $B_{n}$ (see the Introduction)

2.4.2 Model equation in the time space. The NBS $\mathbf{U}_{n}$ on $B_{n}$, which is such that $\hat{\mathbf{U}}_{n}$ is the solution of eqn (30), verifies for a.e. $t \in \mathbb{R}$ the following equation:

$$
\mathbf{M}_{n} \ddot{\mathbf{U}}_{n}(t)+\mathbf{C}_{n} \dot{\mathbf{U}}_{n}(t)+\mathbf{K}_{n} \mathbf{U}_{n}(t)=\mathbf{F}_{n}(t) .
$$

Using Sec. 2.3.1(a), we know that $\mathbf{U}_{n}, \dot{\mathbf{U}}_{n}$, and $\ddot{\mathbf{U}}_{n}$ $\in H_{m}\left(B_{n}\right)$. Equation (34) is called the model equation in the time space. Using the results of Sec. 2.2, we see that $\mathbf{F}_{n}$ and $\mathbf{U}_{n}$ have two time scales: the small time scale $\tau_{c}$ and the large time scale $\tau_{L}$.

\subsection{Actual construction of the model MF equation}

We use a specific method of multiple scales to solve the MF equation (34). (1) The small time scale $\tau_{c}$ is analytically treated by using eqn (30) in the Fourier space. We apply a frequential shift with the value $\Omega_{n}$. Thus, no approximation is introduced in the construction of the solution for the rapid fluctuations with the $\tau_{c}$ time scale. (2) The large time scale $\tau_{\mathbf{L}}$ is directly treated in the time domain. This low frequency part of the solution is given by a time differential equation, called the associated low frequency equation. This equation is solved by using a classical direct step by step numerical integration method. Thus, some usual approximation errors are introduced, but only on the $\tau_{\mathrm{L}}$ scale slow fluctuations of the solution.

2.5.1 Analytical treatment of the rapid fluctuations We apply to eqn (30) the shift defined by $\omega$ $=\omega^{\prime}+\Omega_{n}$, with $\omega^{\prime} \in B_{0}$ and $\omega \in B_{n}$. We obtain for a.e. $\omega^{\prime} \in B_{0}$;

$$
\begin{array}{r}
{\left[-\left(\omega^{\prime}+\Omega_{n}\right)^{2} \mathbf{M}_{n}+i\left(\omega^{\prime}+\Omega_{n}\right) \mathbf{C}_{n}+\mathbf{K}_{n}\right]} \\
\times \hat{\mathbf{U}}_{n}\left(\omega^{\prime}+\Omega_{n}\right)=\hat{\mathbf{F}}_{n}\left(\omega^{\prime}+\Omega_{n}\right) .
\end{array}
$$

Using eqn (18) we see that the LFSs $\mathbf{U}_{0}$ and $\mathbf{F}_{0}$ in $H_{m}\left(B_{0}\right)$, associated with the NBSs $\mathbf{U}_{n}$ and $\mathbf{F}_{n}$ in $H_{m}\left(B_{n}\right)$ are such that, for a.e. $\omega^{\prime}$ in $B_{0}$ and $t$ in $\mathbb{R}$ :

$$
\begin{array}{ll}
\hat{\mathbf{F}}_{0}\left(\omega^{\prime}\right)=\hat{\mathbf{F}}_{n}\left(\omega^{\prime}+\Omega_{n}\right) ; & \mathbf{F}_{0}(t)=\mathrm{e}^{-i \Omega_{n} t} \mathbf{F}_{n}(t), \\
\hat{\mathbf{U}}_{0}\left(\omega^{\prime}\right)=\hat{\mathbf{U}}_{n}\left(\omega^{\prime}+\Omega_{n}\right) ; & \mathbf{U}_{n}(t)=\mathrm{e}^{i \Omega_{n} t} \mathrm{U}_{0}(t) .
\end{array}
$$

Then we have for a.e. $\omega^{\prime}$ in $B_{0}$ :

$$
\left[-\omega^{\prime 2} \mathbf{M}_{n}+i \omega^{\prime} \mathbf{D}_{n}+\mathbf{Z}_{n}\right] \hat{\mathbf{U}}_{0}\left(\omega^{\prime}\right)=\hat{\mathbf{F}}_{0}\left(\omega^{\prime}\right),
$$

where $\mathbf{D}_{n}$ and $\mathbf{Z}_{n}$ are $(m \times m)$ complex symmetric 
matrices:

$$
\begin{aligned}
& \mathbf{D}_{n}=\mathbf{C}_{n}+2 i \Omega_{n} \mathbf{M}_{n}, \\
& \mathbf{Z}_{n}=\mathbf{K}_{n}+i \Omega_{n} \mathbf{C}_{n}-\Omega_{n}^{2} \mathbf{M}_{n} .
\end{aligned}
$$

The LFS $\mathrm{U}_{0}$ on $B_{0}$, solution of eqn. (38) is such that $\mathbf{U}_{0}, \dot{\mathbf{U}}_{0}$, and $\ddot{\mathbf{U}}_{0} \in H_{m}\left(B_{0}\right)$, and verifies for a.e. $t$ in $\mathbb{R}:$

$$
\mathbf{M}_{n} \ddot{\mathbf{U}}_{0}(t)+\mathbf{D}_{n} \dot{\mathbf{U}}_{0}(t)+\mathbf{Z}_{n} \mathbf{U}_{0}(t)=\mathbf{F}_{0}(t) .
$$

Equation (41) is called the low frequency equation in the time domain, associated with the MF equation (34), because the only time scale of $\mathbf{F}_{0}$ and $\mathbf{U}_{0}$ is the large time scale $\tau_{\mathrm{L}}$. We see that eqn (41) can also be obtained in replacing the expression (37) of $\mathbf{U}_{n}(t)$ in eqn (34).

2.5.2 Explicit MF solution as a function of the associated LFS. For any $l \in \mathbb{Z}=\{-\infty, \ldots,-1$, $0,1, \ldots,+\infty\}$ we define the family of functions $\varphi$, on $\mathbb{R}$ such that

$$
t \rightarrow \varphi_{I}(t)=\frac{1}{\sqrt{\tau_{\mathbf{L}}}} \frac{\sin \left[\pi\left(t / \tau_{\mathbf{L}}-l\right)\right]}{\pi\left(t / \tau_{\mathbf{L}}-l\right)} .
$$

The set $\left\{\varphi_{l}, l \in \mathbb{Z}\right\}$ is an orthonormal basis of $H_{1}\left(B_{0}\right)$. Then, considering the Shannon's sampling theorem $[34,79,85]$ for the function $\mathrm{U}_{0} \in H_{m}\left(B_{0}\right)$, and taking into account eqn (37), we obtain for $\mathbf{U}_{m}$ $\in H_{m}\left(\mathrm{~B}_{n}\right)$, and for a.e. $t$ in $\mathbb{R}$ :

$$
\mathbf{U}_{n}(t)=\sqrt{\tau_{\mathbf{L}}} \mathrm{e}^{i \Omega_{n} t} \Sigma_{l \in \mathbb{Z}} \mathbf{U}_{0}\left(l \tau_{\mathrm{L}}\right) \varphi_{l}(t) .
$$

Moreuver, in the Fourier space, we have for a.e. $\omega$ in $\mathbb{R}$ :

$$
\hat{\mathbf{U}}_{n}(\omega)=\tau_{\mathbf{L}} \hat{\mathbf{1}}_{B_{n}}(\omega) \Sigma_{l \in \mathbb{Z}} \mathbf{U}_{0}\left(l \tau_{\mathbf{L}}\right) \mathrm{e}^{-i / \tau_{\mathbf{L}}\left(\omega-\Omega_{n}\right)} .
$$

The expressions of $\hat{\mathbf{U}}_{n}(\omega)$ and $\hat{\hat{U}}_{n}(\omega)$ are given by eqn (44) replacing $\mathbf{U}_{0}\left(l_{\tau_{\mathrm{L}}}\right)$, respectively, by $\mathbf{V}_{0}\left(l \tau_{\mathrm{L}}\right)$ and $\mathbf{W}_{0}\left(l \tau_{\mathrm{L}}\right)$, with

$$
\begin{aligned}
\mathbf{V}_{0}\left(l \tau_{\mathrm{L}}\right) & =\dot{\mathbf{U}}_{0}\left(l \tau_{\mathbf{L}}\right)+i \Omega_{n} \mathbf{U}_{0}\left(l \tau_{\mathbf{L}}\right), \\
\mathbf{W}_{0}\left(l \tau_{\mathbf{L}}\right) & =\ddot{\mathbf{U}}_{0}\left(l \tau_{\mathbf{L}}\right)+2 i \Omega_{n} \dot{\mathbf{U}}_{0}\left(l \tau_{\mathrm{L}}\right)-\Omega_{n}^{2} \mathbf{U}_{0}\left(l \tau_{\mathrm{L}}\right) .
\end{aligned}
$$

The series on the right sides of eqns (43) and (44) are convergent in $H_{m}\left(B_{n}\right)$. The relation (44) determines the MF solution as only a function of the low frequency sampled time solution $\mathbf{U}_{0}$ of eqn (41). In particular the relation (44) determines the f.r.f. $\omega$ $\rightarrow \mathbf{T}(\omega)$ on $B_{n}$ by taking into account the results of Sec. 2.3.1(b). Let $p \in\{1,2, \ldots, m\}$ and let $Q$ be the constant $(p \times m)$ real matrix such that an observation $q_{n}(t)$ of the mechanical system is written as

$$
q_{n}(t)=Q \mathbf{U}_{n}(t) .
$$

Thus, $q_{n}, \dot{q}_{n}$, and $\ddot{q}_{n} \in H_{p}\left(B_{n}\right)$ and for a.e. $\omega$ in $\mathbb{R}$ we have

$$
\hat{q}_{n}(\omega)=\tau_{\mathbf{L}} \hat{\mathbf{1}}_{B_{n}}(\omega) \Sigma_{l \in \mathbb{Z}} Q \mathbf{U}_{0}\left(l \tau_{\mathbf{L}}\right) \mathrm{e}^{-i \tau_{\mathbf{L}}\left(\omega-\Omega_{n}\right)} .
$$

Likewise, we obtain $\hat{\dot{q}}_{n}(\omega)$ and $\hat{\ddot{q}}_{n}(\omega)$ by replacing into eqn (48), $\mathrm{U}_{0}$ by $V_{0}$ and $W_{0}$.

Moreover, the energy of $q_{n}$, given by

$$
E_{q}\left(B_{n}\right)=\|q\|\left\|_{p}^{2}=\frac{1}{2 \pi} \int_{B_{n}}\right\| \hat{q}_{n}(\omega) \|_{p}^{2} \mathrm{~d} \omega
$$

is written as

$$
E_{q}\left(B_{n}\right)=\tau_{\mathbf{L}} \Sigma_{l \in \mathbb{Z}}\left\|Q \mathbf{U}_{0}\left(l_{\mathbf{L}}\right)\right\|_{p}^{2} .
$$

If we write $B_{n}=\cup_{i=1}^{I} B_{n, i}$, the energy $E_{q}\left(B_{n, i}\right)$ is given by eqn (49), but cannot be calculated by eqn (50). One must calculate $E_{q}\left(B_{n, i}\right)$ by using eqns (49) and (48) with the frequency integration on $B_{n, i}$. In the same way we have similar results for $\dot{q}_{n}$ and $\ddot{q}_{n}$, replacing into eqns (49) and (50), $q_{n}$ by $\dot{q}_{n}$ or $\ddot{q}_{n}$, and $\mathrm{U}_{0}$ by $V_{0}$ or $W_{0}$. Note that there are two possibilities for the representation of the square norm of the f.r.f. of $q_{n}$ on $B_{n}$ : (1) a high frequency resolution is obtained by calculating $\omega \rightarrow\left\|\hat{q}_{n}(\omega)\right\|_{p}^{2}$ with eqn (48) for a.e. $\omega$ in $B_{n}$. (2) A representation per frequency band is given by studying $i \rightarrow$ $E_{q}\left(B_{n, i}\right)$.

In the applications of the part II, we have chosen the second representation. The bands $B_{n}$ are fixed and inside each band $B_{n}$, the frequency evolution is given by $i \rightarrow E_{q}\left(B_{n, i}\right)$ or $i \rightarrow 10 \log _{10} E_{q}\left(B_{n, i}\right)$.

2.5.3 Numerical treatment of the slow fluctuations. Let $\mathbf{U}_{0} \in H_{m}\left(B_{0}\right)$ be the LFS, solution of eqn (38), or equivalently of eqn (41). Thus one can easily prove that, for any $\epsilon>0$, there are two finite real constants $t_{\mathbf{I}}(\epsilon)<0$ and $t \mathrm{~F}(\epsilon)>0$ such that

$$
\text { II } \mathbf{U}_{0}-\mathrm{U}_{0} \|_{m} \leqslant \epsilon,
$$

where $\mathbf{U}_{0}$ is the unique solution belonging to $H_{m}$ of the following problem:

$$
\begin{aligned}
& \mathbf{M}_{n} \ddot{\sim}_{0}(t)+\mathbf{D}_{n}{\underset{\sim}{\mathbf{U}}}_{0}(t)+\mathbf{Z}_{n}{\underset{\sim}{\mathbf{U}}}_{0}(t)=\mathbf{F}_{0}(t), \\
& t \in] t_{\mathbf{I}}(\epsilon), t_{\mathrm{F}}(\epsilon)[, \\
& \underline{\sim}_{0}(t)=\dot{\mathbf{U}}_{0}(t)=0 \text { for any } t \leqslant t_{\mathbf{I}}(\epsilon) \text { and } t \geqslant t_{\mathbf{F}}(\epsilon) \text {. }
\end{aligned}
$$

It is obvious that $\left\|\mid \mathbf{U}_{0}-\mathbf{U}_{0}\right\|_{m} \rightarrow 0$ when $t_{\mathbf{I}}(\boldsymbol{\epsilon})$ $\rightarrow-\infty$ and $t_{\mathrm{F}}(\epsilon) \rightarrow+\infty$.

In these conditions we can build a numerical approximation of eqn (41) by solving the problem (52) which is a standard low frequency problem. We can thus use any direct unconditionally stable step by step time integration algorithm: Newmark, $\theta$-Wilson method. 
(a) As the highest frequency existing in the LFS $\mathbf{F}_{0}$ and $\mathbf{U}_{0}$ is $\Delta \omega / 2$, the integration time step, denoted by $\Delta t$, must be less than the sampling time $\tau_{\mathrm{L}}$. To use formulas (43), (44), (48), and (50), $\mathbf{U}_{0}(t)$ must be known at instants $l \tau_{\mathrm{L}}, l \in \mathbb{Z}$. Consequently, $\Delta t$ will be such that $\Delta t=\tau_{\mathrm{L}} / \mathbf{L}_{\mathrm{T}}$ where $\mathbf{L}_{\mathrm{T}}$ is a positive integer greater than 1 . The choice of $\mathbf{L}_{\mathbf{T}}$ partially conditions the precision that will be obtained in the solution and depends only on the numerical method of integration used. For instance, with the Newmark method $\mathbf{L}_{\mathbf{T}}=3$ is a good value.

(b) We let $t_{1}=-\mathbf{L}_{\mathrm{T}} \tau_{\mathbf{L}}$ and $t_{\mathbf{F}}=\mathbf{L}_{\mathbf{F}} \tau_{\mathbf{L}}$, where $\mathbf{L}_{\mathbf{I}}$ and $\mathbf{L}_{\mathbf{F}}$ are positive integers. The initial and final instants $t_{\mathbf{I}}$ and $t_{\mathrm{F}}$ are thus defined by $\mathbf{L}_{\mathbf{I}}$ and $\mathbf{L}_{\mathrm{F}}$. The selection of $\mathbf{L}_{\mathbf{l}}$ is related to the part of the input energy truncated. As $\mathbf{F}_{0} \in H_{m}\left(B_{0}\right)$, for any $\epsilon>0$, finite $\mathbf{L}_{\mathbf{I}}$ exists such that $\int_{-\infty}^{-\mathbf{L}_{\mathbf{1}} \mathbf{I}^{\top} \mathbf{L}}\left\|\mathbf{F}_{0}(t)\right\|_{m}^{2} \mathrm{~d} t \leqslant \epsilon$. Thus, the input energy truncated can be made as small as desired. For instance, $\mathbf{L}_{\mathbf{I}}=3$ or 5 is a good value for the excitation defined in Sec. 2.3.1(b).

(c) As ${\underset{\sim}{0}}_{0} \in \mathbb{H}_{m}$, again, for any $\epsilon>0$, finite $\mathbf{L}_{\mathbf{F}}$ exists such that $\int_{\mathbf{L}_{\mathbf{F}} \tau_{\mathbf{L}}}^{+\infty}\left\|{\underset{\sim}{0}}_{0}(t)\right\|_{m}^{2} \mathrm{~d} t \leqslant \epsilon$. Thus, the energy not taken into account in the interval $\left[t_{\mathbf{F}}\right.$, $+\infty$ [, can be made as small as wanted. The choice of $\mathbf{L}_{\mathbf{F}}$ is directly related to the dynamics of the system governed by the damping matrix $\mathbf{C}_{n}$. As the total energy introduced is known before integration is started, and as at each instant $t>t_{1}$, the total energy dissipated during the time interval $t-t_{\mathrm{I}}$ can be determined, the energy budget makes it possible to determine $\mathbf{L}_{\mathbf{F}}$ automatically to obtain a given precision of the solution. Let us note that, in the MF domain, $\Omega_{n}$ is large enough. Thus, even a very slightly damped system, the function $t \rightarrow$ $\left\|\mathbf{U}_{n}(t)\right\|_{m}=\left\|\mathbf{U}_{0}(t)\right\|$ is rapidly decreasing. For instance, $\mathbf{L}_{\mathbf{F}}=7$ or 9 is a good value for the following case: (1) the excitation is defined in Sec. 2.3.1(b), (2) $\Delta \omega / \Omega_{n}=1 / 20$, (3) the equivalent mean damping rate on the band $B_{n}$ is $\xi_{n}=0.005$.

(d) The relations (43), (44), (48), and (50) use a sum of every instant $l_{\mathrm{L}}, l \in \mathbb{Z}$. The energy considerations discussed in points (b) and (c) above, directly give the truncations to be carried out on the sums $\Sigma_{l \in \mathbb{R}}$. The $\Sigma_{l \in \mathbb{Z}}$ need only replaced by $\Sigma_{l}^{L_{F}}=-\mathbf{L}_{1}$.

(e) As a final remark, let us indicate that for all the presented applications in part II, the calculations have been realized for each narrow band $B_{n}$ with a total number of integration time step: $\mathbf{L}_{\mathbf{T}}\left(\mathbf{L}_{\mathbf{I}}\right.$ $+L_{F}$ ) of the order of $30-40$. For this small number, using eqn (48), we obtain all the dynamics in the MF band $B_{n}$ with a very good precision.

\subsection{Implantation of the MF method in ADINA}

2.6.1 General considerations. For a fixed band $B_{n}$, we must (1) solve problem (52) and, (2) calculate the quantities such as eqns (43), (44), (48), (49), and (50). Note that the matrices $\mathbf{D}_{n}$ and $\mathbf{Z}_{n}$, defined by eqns (39) and (40) are $(m \times m)$ complex, symmetric, and that the initial band or skyline structure of the matrices $\mathbf{M}_{n}, \mathbf{C}_{n}$, and $\mathbf{K}_{n}$ is kept for $\mathbf{D}_{n}$ and $\mathbf{Z}_{n}$. Thus, the implantation of the MF method, in a general finite element program changes neither the fundamental structure of the program nor the dynamic allocation of the core and the $\mathrm{I} / \mathrm{O}$ management. We must only complexify the step by step time integration algorithm. We are going to specify this point. Let us consider the step by step solution of eqn (52) using the Newmark integration method. Let $a_{0}, a_{1}, \ldots, a_{7}$ be the real constants of the Newmark's algorithm. This algorithm is the following:

(a) Initial calculations: (1) Form the effective ma$\operatorname{trix} \mathbf{H}=\mathbf{Z}_{n}+a_{0} \mathbf{M}_{n}+a_{1} \mathbf{D}_{n}$. Using eqns (39) and (40) we obtain

$$
\mathbf{H}=\mathbf{K}_{n}+b_{0} \mathbf{M}_{n}+b_{1} \mathbf{C}_{n}
$$

with $b_{0}=a_{1}+i \Omega_{n}$ and $b_{1}=a_{0}+2 i \Omega_{n} a_{1}-\Omega_{n}^{2}$ are two complex constants. (2) Triangularize the complex effective matrix: $\mathbf{H}=\mathbf{L} \mathbf{D ~ L}^{T}$ where $\mathbf{L}$ and D are complex matrices.

(b) For each time step: (1) Calculate effective loads at time $t+\Delta t: \mathbf{R}(t+\Delta t)=\mathbf{F}_{0}(t+\Delta t)+$ $\mathbf{M}_{n}\left[a_{0} \mathbf{U}_{0}(t)+a_{2} \dot{\mathbf{U}}_{0}(t)+a_{3} \ddot{\mathbf{U}}_{0}(t)\right]+\mathbf{D}_{n}\left[a_{1} \mathbf{U}_{0}(t)+\right.$ $\left.a_{4} \dot{\mathrm{U}}_{0}(t)+a_{5} \ddot{\mathrm{U}}_{0}(t)\right]$. Using eqn (39) we obtain

$$
\begin{array}{r}
\mathbf{R}(t+\Delta t)=\mathbf{F}_{0}(t+\Delta t)+\mathbf{M}_{n}\left[b_{2} \mathbf{U}_{0}(t)\right. \\
\left.+b_{3} \dot{\mathrm{U}}_{0}(t)+b_{4} \ddot{\mathbf{U}}_{0}(t)\right]+\mathbf{C}_{n}\left[a_{1} \mathrm{U}_{0}(t)\right. \\
\left.+a_{4} \dot{\mathrm{U}}_{0}(t)+a_{4} \ddot{\mathrm{U}}_{0}(t)\right]
\end{array}
$$

where $b_{2}=a_{0}+2 i \Omega_{n} a_{1}, b_{3}=a_{2}+2 i \Omega_{n} a_{4}, b_{4}=$ $a_{3}+2 i \Omega_{n} a_{5}$ are complex constants. (2) Solve for displacements at time $t+\Delta t$ :

$$
\mathbf{L} \mathbf{D} \mathbf{L}^{\mathrm{T}} \mathbf{U}_{0}(t+\Delta t)=\mathbf{R}(t+\Delta t) \text {. }
$$

(3) Calculate accelerations and velocities at time $t$ $+\Delta t$ :

$$
\begin{aligned}
\ddot{\mathbf{U}}_{0}(t+\Delta t)=a_{0}\left[\mathbf{U}_{0}(t+\Delta t)-\mathbf{U}_{0}(t)\right] & +a_{2} \dot{\mathbf{U}}_{0}(t)-a_{3} \ddot{\mathbf{U}}_{0}(t), \\
& \\
\dot{\mathbf{U}}_{0}(t+\Delta t)=\dot{\mathbf{U}}_{0}(t)+ & a_{6} \ddot{\mathbf{U}}_{0}(t)+a_{7} \ddot{\mathbf{U}}_{0}(t+\Delta t) .
\end{aligned}
$$

Thus we see that the fundamental structure of the Newmark algorithm is not modified. This conclusion is true for other algorithms such as the $\theta$-Wilson algorithm. One must only:

(1) Realize the assemblage of the effective matrix $\mathbf{H}$ [eqn (53)] with a complex form.

(2) Complexify the subroutine which realizes (i) the factorization of $\mathbf{H}$, (ii) the reduction of the righthand side effective load vector and the back substitution [eqn (55)].

(3) Complexify the calculation of the effective loads [eqn (54)] and the calculation of the acceleration and velocity [eqn (56)]. Note that the substructuring capabilities are kept. 
2.6.2 Implantation in ADINA. We have developed at ONERA this MF method in ADINA[3].

(A) we have added the MF linear dynamic analysis. In order to do so we have realized the modifications described in Sec. 2.6.1 with the Newmark method and we have added the algorithms to compute eqns (44), (48)-(50).

(B) Moreover, for our applications (see part II) we had to increase the capabilities in three directions:

(a) Take into account a consistent damping matrix $\mathbf{C}_{n}$ generated by finite elements. We need a consistent damping matrix (1) for the viscoelastic materials, (2) for the viscous compressible fluids occupying a bounded domain of the space, and (3) for hydrodynamical operator relating to the hydroelasticity with a perfect compressible unbounded fluid.

(b) Take into account a multiple load vector, i. e. for each time step: (1) build simultaneously $J$ effective loads $\mathbf{R}_{j}(t+\Delta t)$ due to a multiple excitation $\mathbf{F}_{0 j}, j \in\{1,2, \ldots, J\}$ [eqn (54)], (2) compute simultaneously $\mathbf{U}_{0, j}(t+\Delta t), j \in\{1, \ldots, J\}$ with eqn (55), and (3) calculate simultaneously eqn (56) for all the $j$ 's. We need this possibility (1) for random excitations (see Sec. 2.3.3); for instance we use this case to study the MF vibrations of an elastic structure excited by the hydrodynamical turbulent boundary layer; (2) in the developments relating to the probabilistic modelization of the structural fuzzy in the complex structures.

(c) Increase the finite element library. This point will be discussed for each new finite element in part II.

(d) Note that for all these developments we have maintained the capabilities of ADINA, i.e. (1) analysis with substructures, (2) the linear constraint equations, (3) the access to the whole library of finite elements.

(e) Two new versions have been developed. A scalar version on a CYBER $170 / 750$ computer, and an optimized vectorial version on a CRAY 1 computer. The CPU gain factor between scalar and vectorial version is satisfactory. For large systems the gain factor can be 20 .

\section{PART II: APPLICATIONS}

3.1 Linear MF vibrations of elastic structures in vacuo

3.1.1 Setting the problem. We consider the linear MF vibrations of an elastic, slightly damped, heterogeneous anisotropic structure in vacuo, occupying a bounded domain in $\mathbb{R}^{3}$ space. The first characteristic of the MF range is that the response brings a very large number of high order vibration eigenmodes. The modal density can be high $a$ priori. On the other hand, for this range we can obtain modal behaviors as in LF, locally or not, as well as global dynamic behaviors, grouping by eigenmode packages when the modal density is large enough. All of the behaviors may appear simultaneous at certain frequencies in the MF broadband considered. That is the case, for instance, for a shell with stiffeners that we will study hereafter. We are going to specify this point. Let us consider the vibratory response of an elastic structure subjected to a point force. In the LF domain the vibratory energy propagates broadly into the structure because the first eigenmodes are generally global. Thus the vibratory energy is not localized around the point where is located the excitation force. In the MF domain, (1) if the modal density is locally small in a MF narrow band we can obtain a similar phenomenon. Generally there is a propagation of the energy for this narrow band, (2) but if the modal density is locally high in this MF narrow band, the vibratory energy is localized around the excitation force. The energy does not propagate into the structure. The concrete example that we present hereafter, shows that the MF situation is complicated because, for heterogeneous structures, the modal density has rapid variations in the MF range.

3.1.2 Theoretical complements. For this case, the theoretical complements are reduced to hardly anything, because the linear elasticity theory is standard.

3.1.2(a) Modelization. For an heterogeneous anisotropic linear elastic structure we use the standard linear elastic finite elements: truss, beam, plate, thin and thick shell, 2D and 3D solid elements. In these conditions, the consistent mass matrix $\mathbf{M}_{n}$ and the stiffness matrix $\mathbf{K}_{n}$ defined by eqn (31) are independent of the frequency and are denoted by $\mathbf{M}_{S}$ and $\mathbf{K}_{S}$. In the MF range, the damping in the materials is globally introduced in writing the damping matrix as

$$
\mathbf{C}_{n}=\lambda_{n} \mathbf{M}_{S}+\mu_{n} \mathbf{K}_{S}
$$

with two possibilities for the constants $\lambda_{n}$ and $\mu_{n}$ : (1) $\lambda_{n}=2 \xi_{n} \Omega_{n}$ and $\mu_{n}=0$. Thus $\xi_{n}$ is the mean loss rate of the structural damping on the MF band $B_{n}$. this means that the critical loss rate of an eigenmode of eigenfrequency $\omega_{j}$ in the band $B_{n}$ is $\xi_{j}=$ $\xi \Omega_{n} \omega_{j}^{-1}$. The damping will be approximatively uniform on the band $B_{n}$, because $\Omega_{n}^{-1} \Delta \omega \ll 1$. (2) $\lambda_{n}=\xi_{n} \Omega_{n}$ and $\mu_{n}=\xi_{n} \Omega_{n}\left(\Omega_{n}^{2}-\Delta \omega^{2} / 4\right)^{-1}$. Thus the $\xi_{j}$ value is such that $\xi_{j} \sim \xi_{n}$ for $\Omega_{n}^{-1} \Delta \omega \ll 1$. In this case the damping is uniform on the narrow band $B_{n}$, but, $\xi_{n}$ can be modified when the band $B_{n}$ is changed.

Note that the situation will be very different for viscoelastic materials: the $\mathbf{C}_{n}$ matrix will be generated by finite elements (see Sec. 4).

We suppose that the boundary conditions are such that the hypotheses introduced in part I are verified.

3.1.2(b) Special treatment of $3 D$ structures with axisymmetric parts. Let us consider a 3D structure, a large part of which is formed with axisymmetric bodies, but the global structure is not axisymmetric. We can modelize such a structure with $3 \mathrm{D}$ finite elements. But, for a given number of DOF, we ob- 
tain a better approximation in modelizing the axisymmetric parts with axisymmetric finite elements. Thus we use this technique when we have this situation. An axisymmetric body is described in the cylindrical coordinates $(r, \theta, z)$ where $z$ is the revolution axis. The radial, axial and tangential components of the displacement field are written as

$$
\begin{aligned}
u_{r}(r, \theta, z, t)= & \Sigma_{N \geqslant 0}\left[u_{N, S}(r, z, t)\right. \\
& \left.\cos N \theta+u_{N, A S}(r, z, t) \sin N \theta\right], \\
u_{z}(r, \theta, z, t)= & \Sigma_{N \geqslant 0}\left[v_{N, S}(r, z, t)\right. \\
& \left.\cos N \theta+v_{N, A S}(r, z, t) \sin N \theta\right], \\
u_{\theta}(r, \theta, z, t)= & \Sigma_{N \geqslant 0}\left[w_{N, S}(r, z, t)\right. \\
& \left.\sin N \theta+w_{N, A S}(r, z, t) \cos N \theta\right],
\end{aligned}
$$

where $\left(u_{N, S}, v_{N, S}, w_{N, S}\right)$ and $\left(u_{N, A S}, v_{N, A S}, w_{N, A S}\right)$ are, respectively, the symmetric and antisymmetric fields of the meridian section and where $N$ is the circumferential node pattern. The methodology is the following: (1) the 3D part is modelized with finite elements. (2) Each axisymmetric part is modelized with axisymmetric finite elements. So we consider $\mathcal{N}$ values of $N$ and for each $N$ we introduce the symmetric and/or antisymmetric DOF's. (3) The whole $3 \mathrm{D}$ structure is assembled by using linear constraint equations defined by eqn (58).

3.1.3 Corresponding development in ADINA.

(a) For the linear MF dynamic analysis, the damping matrix can be generated by eqn (57). When substructures are used, the $\mathbf{C}_{n}$-damping matrix can be different in each substructure.

(b) We have implanted in the $2 \mathrm{D}$ solid elements, the element type: "axisymmetric $N \geqslant 1$ " which can be used (1) for isotropic and orthotropic linear elastic material, and (2) for other materials (see Secs 3 and 4).

3.1.4 Validation. We present a very simple example which allows to validate the MF algorithm. We consider a thin circular cylindrical shell with a constant section. The conditions are the shear diaphragms at both ends. For this structure an analytical solution is known. The data (in SI units) are the following: length 0.863 , thickness 0.008 , radius 0.25 , material: isotropic steel, $E=0.21 \times 10^{12}, \nu$ $=0.3, \rho=7850, \xi_{n}=0.003$. We use the excitation $\mathbf{F}_{n, j}$ defined in Sec. 2.3.1(b) on the band $B_{n}=[2000$ $2100] \mathrm{Hz}$. The spatial part $A_{j}$ is a concentrated force located in the central plane of symmetry. Taking into account one of the two planes of symmetry, only a half cylinder is meshed in $3 \mathrm{D}$ with $(10 \times 11)$ nine nodes thin shell elements. A reduced integration scheme is used. The model has 2205 DOFs and the MF integration parameters are $\mathbf{L}_{\mathrm{T}}=3, \mathbf{L}_{\mathbf{l}}$ $=4, \mathbf{L}_{\mathbf{F}}=9$. The observations $q_{n}$ are the radial displacements at the nodes located on the generatrix and on the half-section which contains the applied force. The calculated quantities are (1) the f.r.f. of the radial displacement at the excitation point, (2) the energy $E_{q}\left(B_{n}\right)$ of $q_{n}$ [eqn (50)]. The
ADINA results are compared with the theory of cylinders on Figs. 1(a) to 1(c). The comparison is satisfactory.

3.1.5 MF local effects in 3D stiffened shell cylinder. In this section we give an example of the local effects in linear dynamic analysis of a structure in the MF range. The given experimental results, which have been made at ONERA are extracted from Ref. [91]. The structure is a steel circular shell cylinder with 56 inside transverse stiffeners (all the stiffeners are not the same), with a floor in the cen-

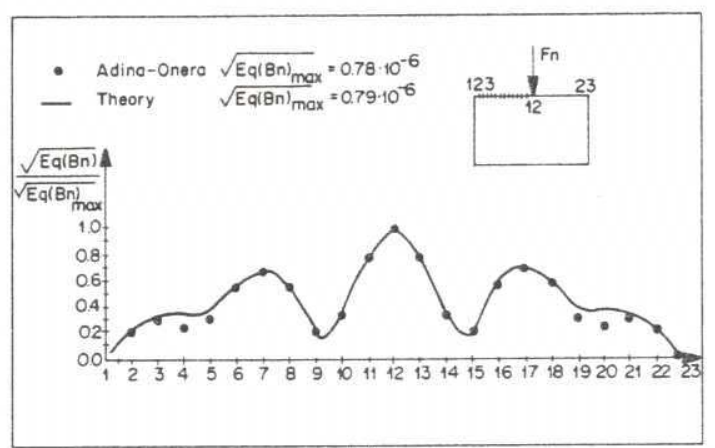

(a)

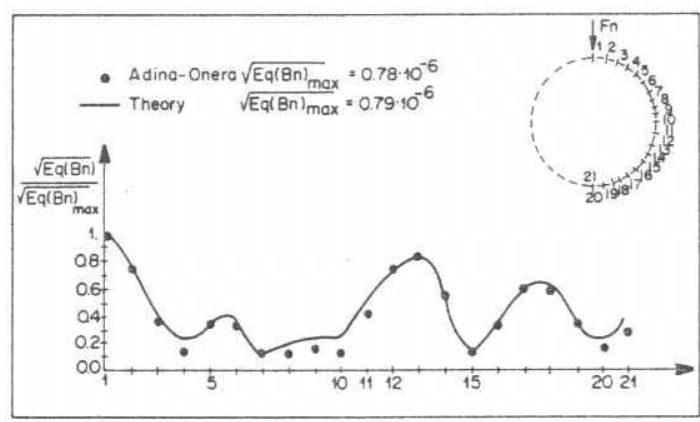

(b)

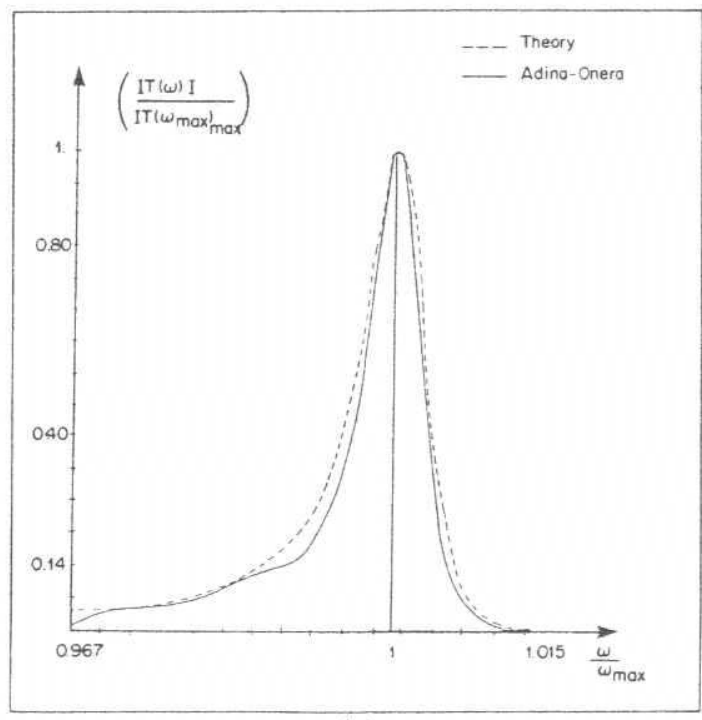

(c)

Fig. 1. Simple example for the validation. Thin circular cylindrical shell. Band $B_{n}=[2000-2100] \mathrm{Hz}$. (a) Longitudinal propagation of energy; (b) circumferential propagation of energy; (c) frequency response function of the excitation point. 
tral ferrule, with two bottoms and with internal subsystems. This structure is a 3D linear elastic medium with an axisymmetric part. The geometry and material properties are defined by Fig. 2(a). The internal subsystems are modelized but are not represented on this figure. The MF dynamic calculations are realized for the broadband $B=[0-3600]$ $\mathrm{Hz}$. This band is partitioned into $36 \mathrm{MF}$ narrow bands $B_{n}$ of bandwidth $100 \mathrm{~Hz}$. For each band $B_{n}$, we apply the excitation $\mathbf{F}_{n, j}$ defined in Sec. 2.3.1(b). The spatial part $\mathbf{A}_{j}$ is a concentrated force located in the central plane of symmetry [see Fig. 2(b)]. The observations $\ddot{q}_{n}$ are the radial accelerations at some points of the shell located (1) on the inside generatrix of the shell and (2) in the three half-crowns $M, N$, and $P$ [see Fig. 2(b)]. The modelization is realized with the method described in Sec. 3.1.2(b). The axisymmetric part is meshed with axisymmetric 2D solid elements. We have introduced 14 values of the circumferential node pattern: $N \in\{0,1$, $2, \ldots, 13\}$. The floor and the internal subsystems are modelized in 3D. Taking into account two planes of symmetry, only a quarter of the structure is considered [see Fig. 2(c)]. The total number of DOF is 25 292. The calculations have been made with a CRAY 1S/1000 computer. The parameters are defined in Table 1 . The mass matrix is consistent, the damping matrix is generated by eqn (57). The fundamental eigenfrequency of this mechanical system is around $80 \mathrm{~Hz}$. We give a few results of the complete analysis which has been made[91]. The experimental results concern only the frequency band [2000-3600] Hz. Figures 3(a) and 3(b) show the f.r.f. of the radial acceleration expressed in terms of energy integrated by frequency band of $20 \mathrm{~Hz}$ at two observation points on the shell, located close to the force. These curves are the graphs of $i, n \rightarrow 10 \log _{10} E_{\ddot{q}}\left(B_{n, i}\right)$ which are computed as we have indicated in Sec. 2.5.2. Figures 4(a) to 4 (c) and 5(a) to 5 (d) show the spatial propagation of the normalized energy $\tilde{E}=E_{\ddot{q}}\left(B_{n}\right) / E_{\max }$ or the radial acceleration per $B_{n}$ band, and computed by eqn (50). Figures 4(a) and 4(b) show that, in the LF domain, the vibratory energy propagates broadly into the structure. When the frequency is increasing to reach the MF domain, Figs. 4(c) and 5 (a) to 5 (d) show that the vibratory energy is partially or completely localized around the applied force. A detailed examination of these figures shows that the phenomena are relatively complicated.

3.2 Linear MF vibrations of elastic structures coupled with an external unviscous compressible unbounded fluid

3.2.1 Setting the problem. In this section we study the linear vibrations of an elastic body immersed in an unbounded compressible fluid and excited in the MF range. We are interested to calculate the MF vibration states of the body and the acoustic field radiated by the body in the fluid. In the MF range it is difficult to use a modal representation of the structure for the reason given in the Introduction. This last representation is classically used for the LF range. Thus, we propose a direct coupled representation by using the basis of the finite elements of the elastic body. The matrices of the hydrodynamical coupling and radiating operators are built with an integral equation of the Helmholtz problem for the fluid.

3.2.2 Theoretical complements. The hypotheses on the elastic structure are those of Sec. 3.1 and we use the same method for modelizing the structure. In particular the mass, damping and stiffness matrices $\mathbf{M}_{S}, \mathbf{C}_{S}$, and $\mathbf{K}_{S}$ of the discretized elastic structure verify the hypotheses of part I (Sec. 2.1), The body occupies an open bounded domain $S$ of $\mathbb{R}^{3}$ with smooth boundary $\partial S=\Sigma \cup \Sigma_{1}$. This body

Table 1. In vacuo calculations with CRAY 1 S/1000 for each band $B_{m}$

MTOT $=600000$ words $(64$ bits $)$

NEQ: Number of DOF

NWK: Number of matrix elements (complex word 128 bits)

ISTOH: Maximum block length (complex word 128 bits)

NEQC: Number of DOF to be condensed in a substructure

\begin{tabular}{|c|c|c|c|c|c|}
\hline & NEQ & NWK & ISTOH & NBLOCK & NEQC \\
\hline Substructure 1 & 1183 & 96480 & 84900 & 2 & 1060 \\
\hline Substructure 2 & 1776 & 217545 & 84900 & 3 & 1590 \\
\hline$\vdots$ & : & : & $\vdots$ & $\vdots$ & \\
\hline Substructure 14 & 1776 & 217545 & 84900 & 3 & 1590 \\
\hline Master structure & 3439 & 1397549 & 84904 & 17 & \\
\hline
\end{tabular}

Total number of DOF $=25292$

$N S T E=\mathbf{L}_{\mathbf{T}}\left(\mathbf{L}_{\mathbf{1}}+\mathbf{L}_{\mathbf{F}}\right)=3 *(4+7)=33$

$C P U$ time $=860$ secs

$\mathrm{I} / \mathrm{O}=9.310^{8}$ words (64 bits)

Total for 36 bands $B_{n}(B=[0-3600] \mathrm{Hz})$

CPU time $=30960$ secs

$\mathrm{I} / \mathrm{O}=2.110^{12}$ bits 
(a)

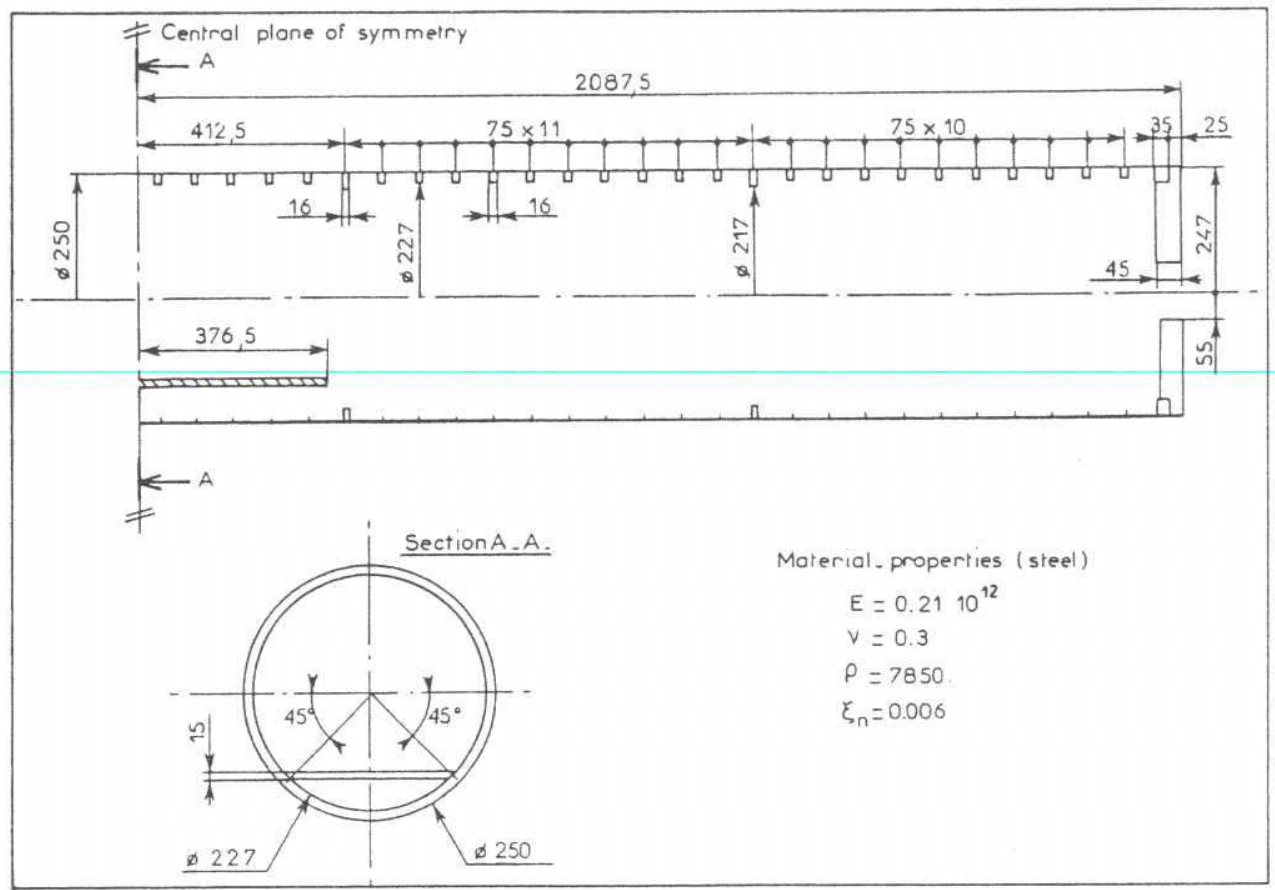

(b)

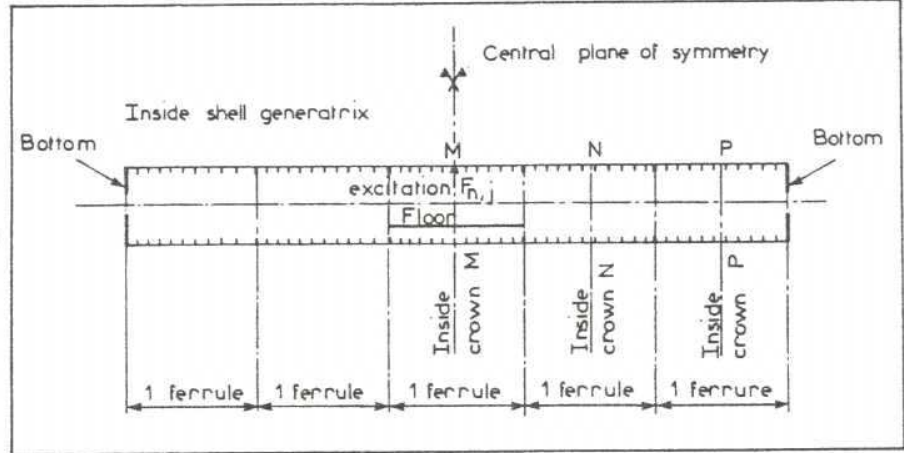

(c)

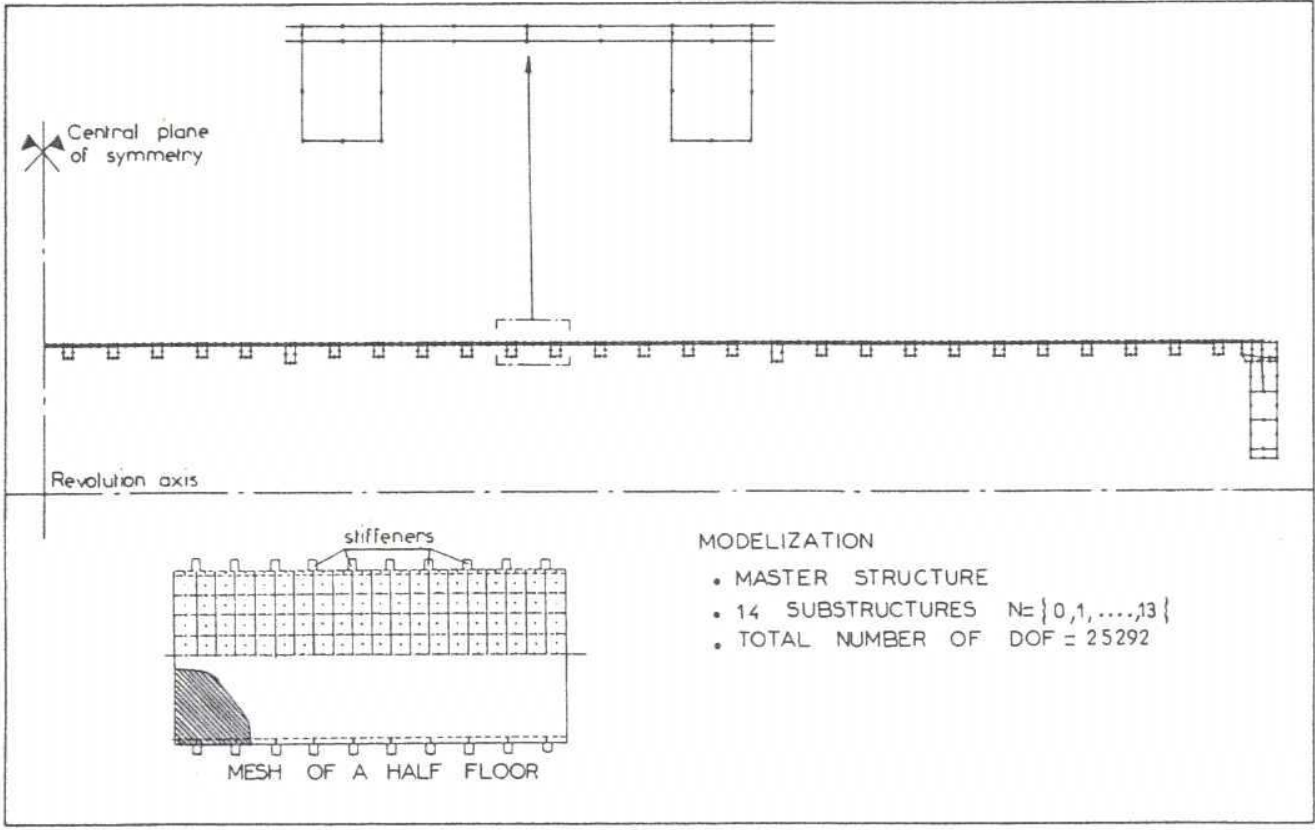

Fig. 2. 3D stiffened shell cylinder with floor in vacuo. (a) Structural geometry and material properties; (b) excitation force and observation points; (c) finite element modelization. 
(a)

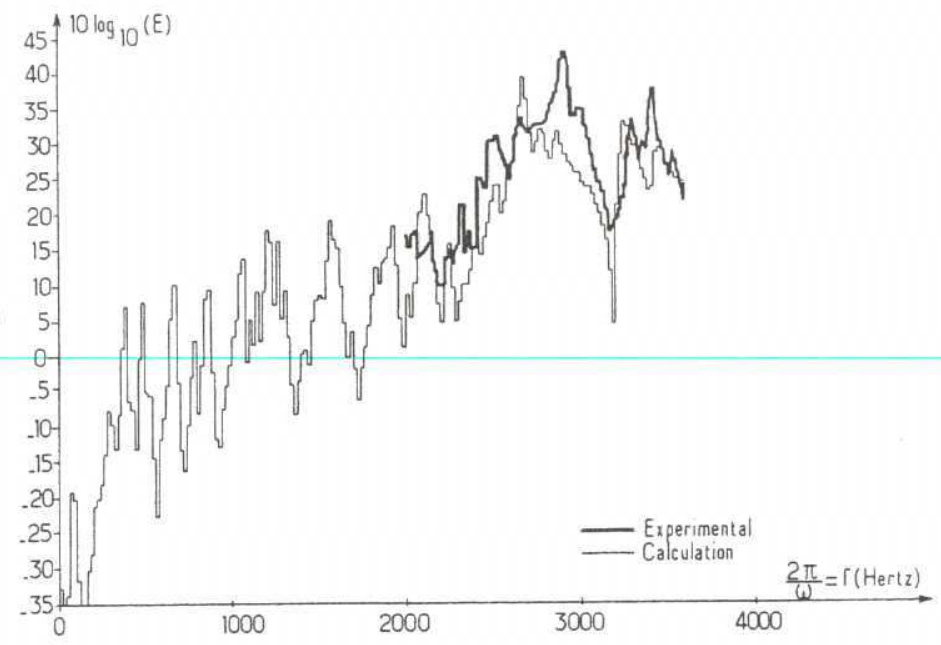

(b)

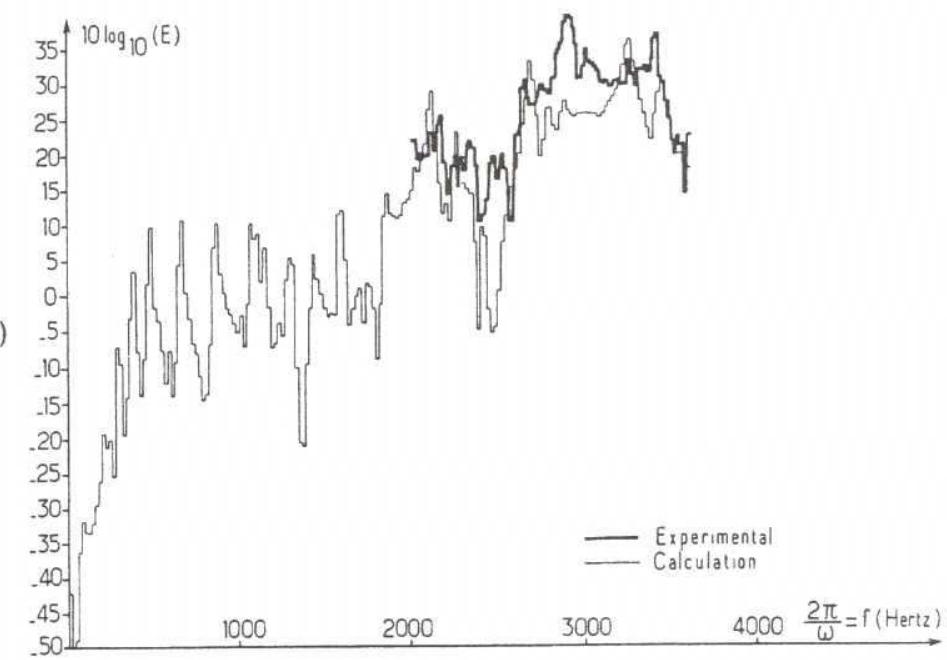

Fig. 3. (a) and (b) Three-dimensional stiffened shell cylinder with floor in vacuo. Frequency response function expressed in terms of energy with experimental comparisons. $E=$ energy of the radial acceleration integrated by frequency band of $20 \mathrm{~Hz}$ at two observations points on the shell.

is immersed in an unviscous compressible dense fluid which occupies an open unbounded domain $L$ of $\mathbb{R}^{3}$ with $\partial L=\Sigma \cup \Sigma_{2}$, where $\Sigma_{2}$ is a free surface (see Fig. 6). If there is no free surface $\Sigma_{2}=\varnothing$. Neglecting the gravity wave on the free surface, we can build an equivalent new problem without free surface. This new problem is defined for the elastic medium $S^{\prime \prime}=S \cup S^{\prime}$ without free surface, where $S^{\prime}$ is the body which is symmetric to $S$ with regard to $\Sigma_{2}$. Consequently, we shall consider $\Sigma_{2}=\varnothing$ henceforth. Note that in the developed computer program we can take into account a free surface which is treated with the previously described method. In these conditions the MF vibration equation of the discretized coupled system, expressed with the only displacement field of the structure, is of the type (29) and is written as

$$
\begin{aligned}
-\omega^{2}\left[\mathbf{M}_{S}+\mathbf{M}_{H}(\omega)\right] & +i \omega\left[\mathbf{C}_{S}+\mathbf{C}_{H}(\omega)\right] \\
& \left.+\mathbf{K}_{S}\right] \hat{\sim}_{m}(\omega)=\hat{\mathbf{F}}_{n}(\omega),
\end{aligned}
$$

where the matrix $\mathbf{B}_{H}(\omega)$ of the hydrodynamical coupling operator is such that

$$
-\omega^{2} \mathbf{B}_{H}(\omega)=-\omega^{2} \mathbf{M}_{H}(\omega)+i \omega \mathbf{C}_{H}(\omega)
$$

The matrices $\mathbf{M}_{H}(\omega)=\operatorname{Re} \mathbf{B}_{H}(\omega)$ and $\mathbf{C}_{H}(\omega)=-\omega$ $\operatorname{Im} \mathbf{B}_{H}(\omega)$ are real symmetric positive definite and classically named the added-mass and radiation damping matrices. We give hereafter a few precisions on the construction of $\mathbf{B}_{H}(\omega)$ and some indications on how to obtain the MF model equation (30) starting from eqn (59).

3.2.2(a) Hydrodynamical coupling and radiating operators. As $\Sigma_{2}=\varnothing$, we have $\partial \mathbf{L}=\Sigma$. Let $x$ be 


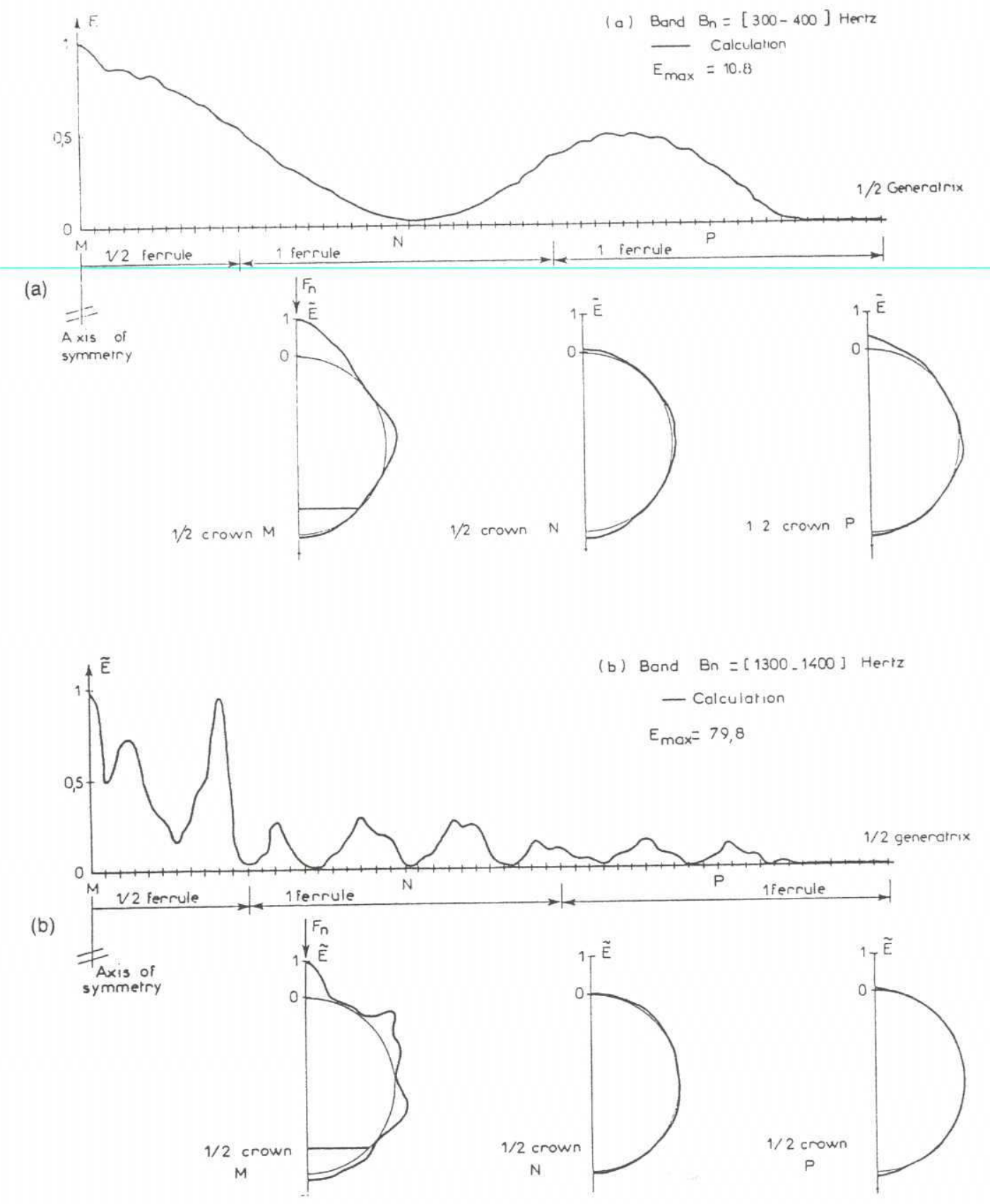

Fig. 4. (a), (b), and (c) Three-dimensional stiffened shell cylinder with floor in vacuo. Spatial propagation of the energy of the radial acceleration $E$ per band $B_{n}$. (Continued on following page.)

a generating point of the space $\mathbb{R}^{3}$ equipped with a Cartesian system $O x_{1} x_{2} x_{3}$. Let $\alpha$ be the unit normal to $\Sigma$ internal to domain $\mathbf{L}$ (see Fig. 7). Let $\rho$ be the mass density of the fluid in its equilibrium state and $c$ the corresponding speed of sound, both assumed to be constant. For any $\omega$ in $\mathbb{R}$, let $x \rightarrow i \omega u(x)$ be a $\mathbb{C}^{3}$-valued harmonic velocity field defined on $\Sigma$ due to the deformation of $\Sigma$. Denoting by $V, \phi$, and $p$, respectively, the harmonic velocity field, velocity potential, and pressure in the fluid, we have

$$
V=\operatorname{grad} \phi ; \quad p=-i \omega \rho \phi .
$$

Let us consider the following Neumann problem related to the Helmholtz equation in the unbounded domain $L$ :

$$
\begin{aligned}
& \Delta \phi+\frac{\omega^{2}}{c^{2}} \phi=0 \text { into } L, \\
& \frac{\partial \phi}{\partial \alpha}=i \omega\langle u, \alpha\rangle_{3} \text { on } \Sigma, \\
& \left|\frac{\partial \phi}{\partial r}-i \frac{\omega}{c} \phi\right|=O\left(1 / r^{2}\right), \\
& |\phi|=O(1 / r), r=\|O x\| \rightarrow+\infty .
\end{aligned}
$$




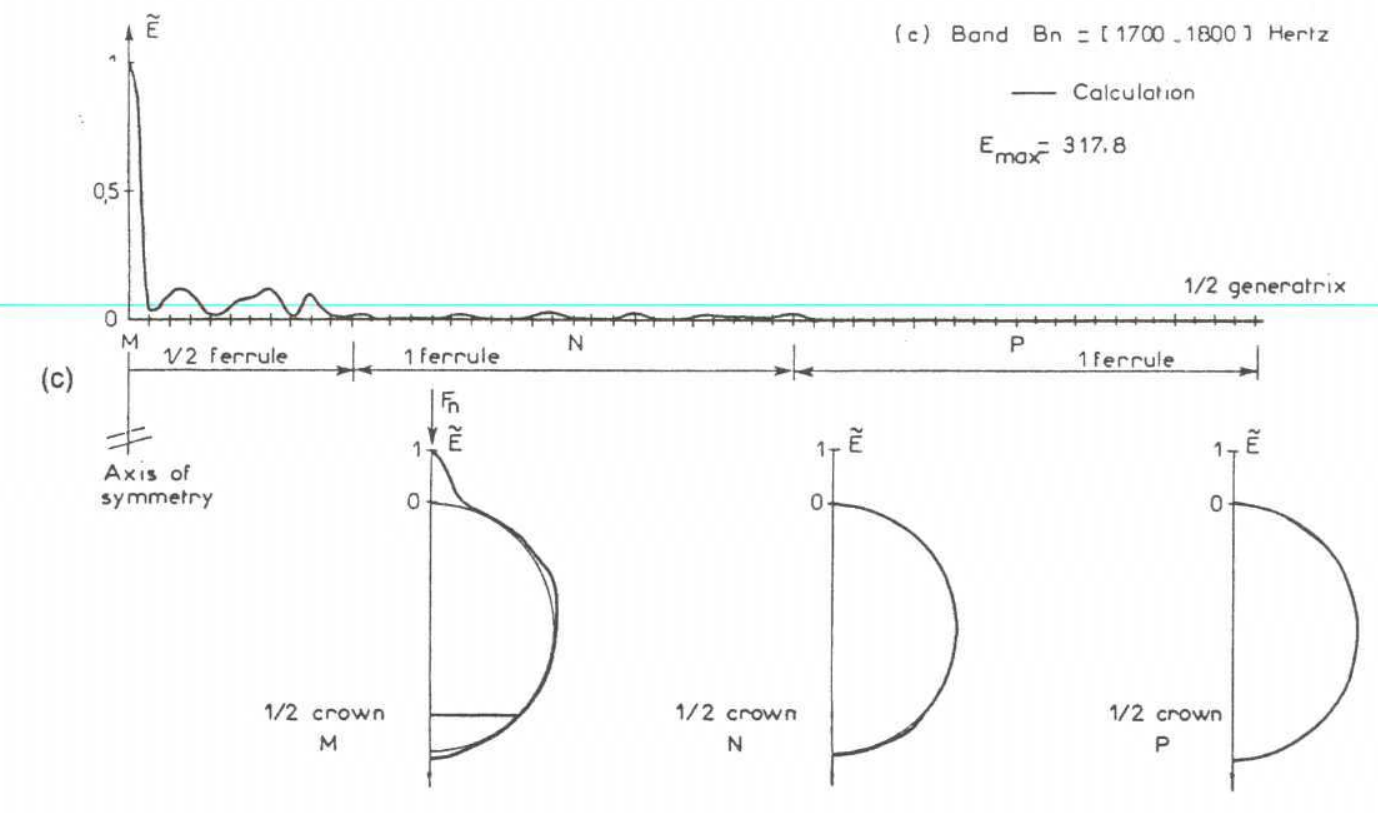

Fig. 4. continued.

Table 2. Hydroelasticity calculations with CRAY $1 \mathrm{~S} / 2000$ for each band $B_{n}$

Step (1): Calculation of $\mathbf{B}_{H}^{\prime}\left(\Omega_{n}\right)$ with FSMFM

NDOFF $=$ number of fluid DOF's $\left(m^{\prime}\right)=1473$

NWKF $=$ number of matrix elements $\mathbf{B}_{H}^{\prime}\left(\Omega_{n}\right)=2169729$ (complex word 128 bits)

CPU time $=1407$ secs

Step (2): Solving the coupled problem with ADINA

MTOT $=580000$ words (64 bits)

NEQ: Number of DOF

NWK: Number of matrix elements (complex word 128 bits)

ISTOH: Maximum block length (complex word 128 bits)

NEQC: Number of DOF to be condensed in a substructure

\begin{tabular}{|c|c|c|c|c|c|}
\hline & NEQ & NWK & ISTOH & NBLOCK & NEQC \\
\hline Substructure 1 & 1357 & 149779 & 43200 & 4 & 1176 \\
\hline Substructure 2 & 2037 & 338541 & 43200 & 8 & 1764 \\
\hline : & $\vdots$ & : & $\vdots$ & $\vdots$ & $\vdots$ \\
\hline Substructure 14 & 2037 & 338541 & 43200 & 8 & 1764 \\
\hline Master structure & 3744 & 2239289 & 42898 & 54 & \\
\hline
\end{tabular}

Total number of DOF $=27852$

NSTE $=\mathbf{L}_{T}\left(\mathbf{L}_{l}+\mathbf{L}_{F}\right)=3 *(4+7)=33$

CPU time $=1555$ secs

Step (3): Acoustic field radiated computed with FSMFR

$\mathrm{CPU}$ time $=307$ secs

Total for one $B_{n}$ band and three steps:

CPU time $=3269$ secs

$\mathrm{I} / \mathrm{O}=2.610^{9}$ words (64 bits)

Total for the complete analysis on $B=[2000-4000] \mathrm{Hz}$

CPU time $=65380$ secs

$\mathrm{I} / \mathrm{O}=3.3 \times 10^{12}$ bits 


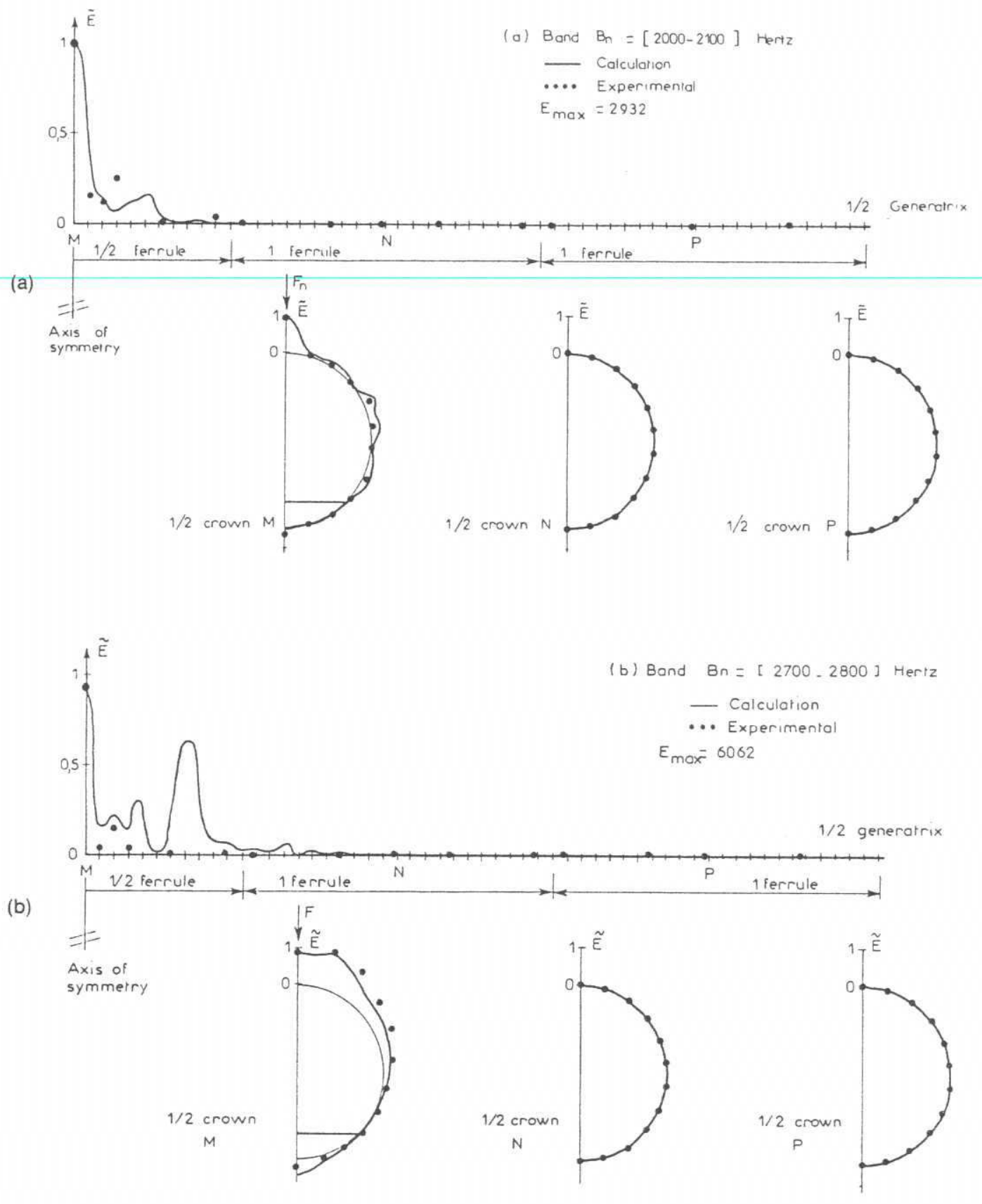

Fig. 5. (a) to (d) Three-dimensional stiffened shell cylinder with floor in vacuo. Spatial propagation of the energy of the radial acceleration $E$ per band $B_{n}$ with experimental comparisons. (Continued on following page.)

If the function $x \rightarrow\langle u(x), \alpha(x)\rangle_{3}$ belongs to an appropriate set of functions, it is known that, for any fixed real $\omega$, the problem (62) has a unique solution $\phi$ which is a $\mathbb{C}$-valued continuous function on $\overline{\mathbf{L}}=$ $\mathbf{L} \cup \Sigma$ (and also on $\mathbb{P}^{3}$ ) such that

$$
\begin{aligned}
\phi_{\Sigma} & =i \omega \tilde{\mathscr{\Re}}_{\omega}\left(\langle u, \alpha\rangle_{3}\right), \\
\forall x \in L, \phi(x) & =i \omega \tilde{\mathscr{R}}_{\omega, x}\left(\langle u, \alpha\rangle_{3}\right),
\end{aligned}
$$

where $\phi_{\Sigma}$ is the potential on $\Sigma, \phi(x)$ the value of the potential at a fixed point $x$ in $L$, and where $\tilde{\mathscr{R}}_{\omega}$ and $\tilde{\mathscr{R}}_{\omega, x}$ are linear operators that must be de- termined. We introduce the notation

$$
[(u, \bar{v})]=\int_{\Sigma}\langle u(x), \overline{v(x)}\rangle_{3} \mathrm{~d} \Sigma(x) .
$$

Let $v$ be a test function belonging to an admissible class of functions. The hydrodynamical coupling operator, denoted by $\Re_{H}(\omega)$, is defined by the equality

$$
\left[\left(f_{H}, \bar{v}\right)\right]=\omega^{2}\left[\left(\mathscr{B}_{H}(\omega) u, \bar{v}\right)\right],
$$

where $f_{H}$ is the hydrodynamical force field applied 

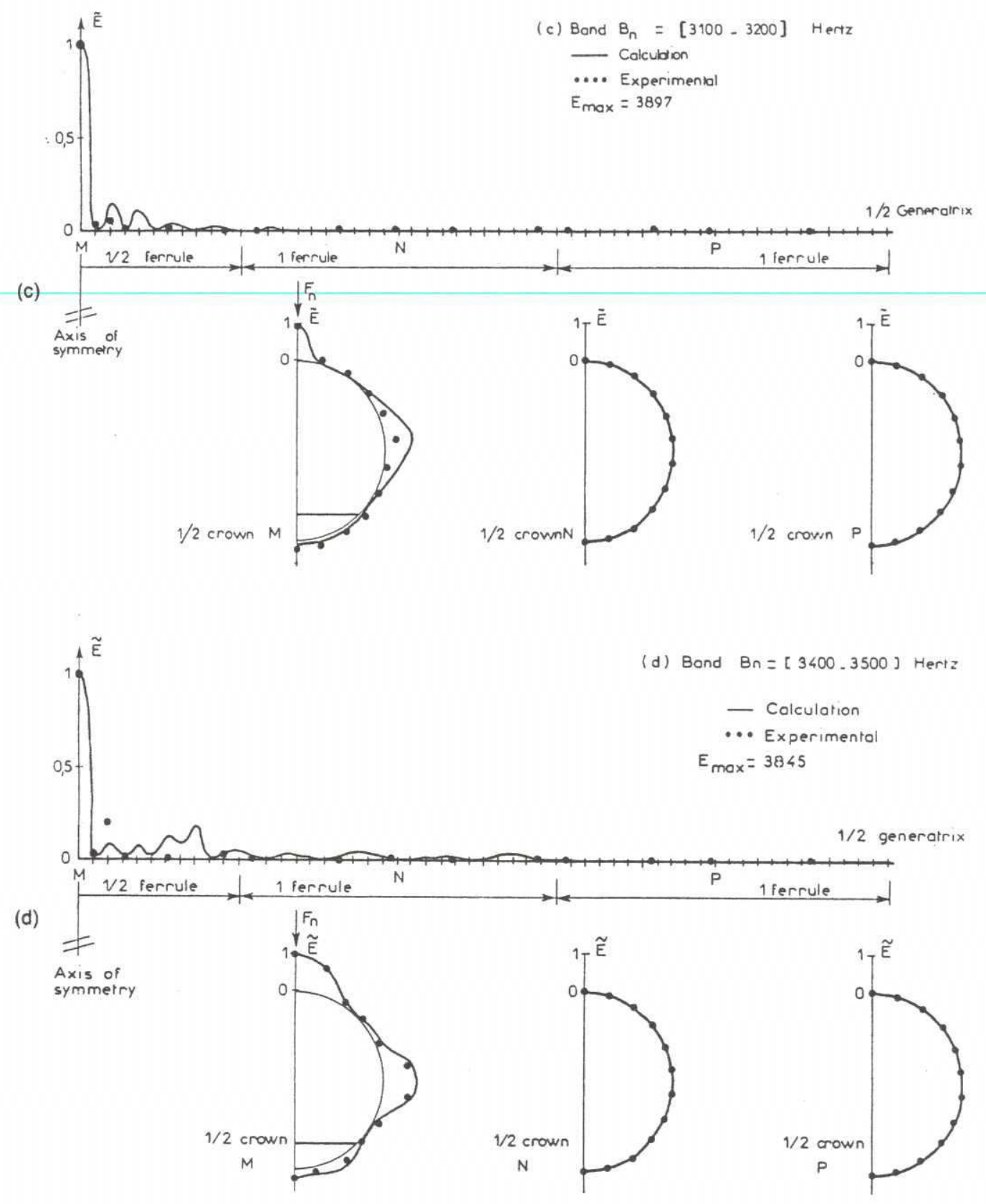

Fig. 5. continued.

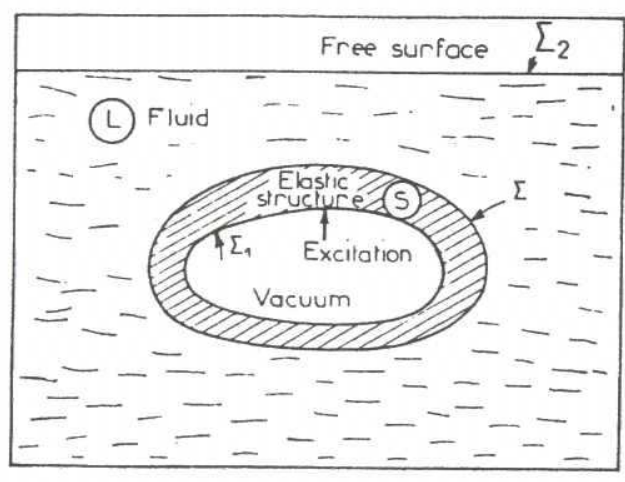

Fig. 6.

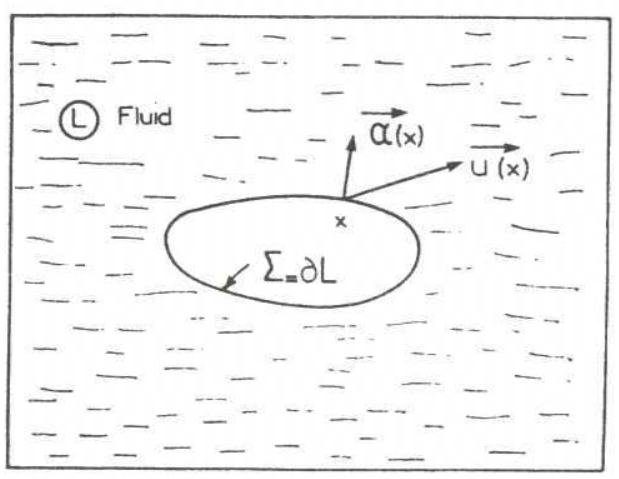

Fig. 7. 
to $\Sigma$ such that

$$
\left[\left(f_{H}, \bar{v}\right)\right]=-\int_{\Sigma} P_{\Sigma}(x)\langle\alpha(x), \overline{v(x)}\rangle_{3} \mathrm{~d} \Sigma(x),
$$

where $P_{\Sigma}$ is the pressure field on $\Sigma$. So, using eqns (61) and (63) we see that the complex symmetric operator $\mathscr{B}_{H}(\omega)$ is defined by the sesquilinear form

$u, v \rightarrow\left[\left(\mathscr{B}_{H}(\omega) u, \bar{v}\right)\right]$

$$
=-\rho \int_{\Sigma}\langle\alpha, \bar{v}\rangle_{3} \tilde{\mathscr{R}}_{\omega}\left(\langle\alpha, u\rangle_{3}\right) \mathrm{d} \Sigma .
$$

In the same way, for any fixed $x$ in $L$, the hydrodynamical radiating operator, denoted by $\mathscr{R}_{H}(\omega, x)$ is defined by the equality

$$
p(x)=-\omega^{2} \Re_{H}(\omega, x) u .
$$

So, using eqns (61) and (64), the linear operator $\Re_{H}(\omega, x)$ is defined by

$$
u \rightarrow \mathscr{R}_{H}(\omega, x) u=-\rho \tilde{R}_{\omega, x}\left(\langle u, \alpha\rangle_{3}\right) .
$$

3.2.2(b) Spatial approximation of the hydrodynamical operators. The surface $\Sigma$ is meshed with isoparametric finite elements which are compatible with the finite elements of the structure (on $\Sigma$ ). Thus, there is a $\left(3 \times m^{\prime}\right)$ real matrix $\mathbf{Q}(x)$ such that for $x \in \Sigma, u(x)=\mathbf{Q}(x) \mathbf{U}^{\prime}$, where $\mathbf{U}^{\prime}$ is the $\left(m^{\prime} \times 1\right)$ column matrix of the $m^{\prime}$ fluid DOFs introduced in the mesh of $\Sigma$. Thus, the $\left(m^{\prime} \times m^{\prime}\right)$ complex symmetric matrix $\mathbf{B}_{H}^{\prime}(\omega)$ and the $\left(1 \times m^{\prime}\right)$ complex matrix $\mathbf{R}_{H}^{\prime}(\omega, x)$ of the operators $\mathscr{B}_{H}(\omega)$ and $\mathscr{R}_{H}(\omega, x)$ are such that

$$
\begin{aligned}
\left\langle\mathbf{B}_{H}^{\prime}(\omega) \mathbf{U}^{\prime}, \overline{\mathbf{V}^{\prime}}\right\rangle & =\left[\left(\Re_{H}(\omega) u, \bar{v}\right)\right], \\
\mathbf{R}_{H}^{\prime}(\omega, x) \mathbf{U}^{\prime} & =\mathscr{R}_{H}(\omega, x) u .
\end{aligned}
$$

If there is a unit normal to $\Sigma$ at a fluid node, we use only one fluid DOF for this node by taking an appropriate local coordinate system. If it is not the case, we have three fluid DOFs at this node. This method allows us to obtain the smallest number of fluid DOF. For the finite element program which solves the coupled equation, the matrix $\mathbf{B}_{H}^{\prime}(\omega)$ is seen by this program as the matrix of a special finite element having $m^{\prime}$ DOFs. This matrix is assembled with the matrices of the finite elements of the elastic structure. Thus in eqn $(60)$, we have denoted by $\mathbf{B}_{H}(\omega)$ the hydrodynamical coupling matrix $\mathbf{B}_{H}^{\prime}(\omega)$ assembled with the matrices of elastic structure. Note that the matrix $\mathbf{B}_{H}^{\prime}(\omega)$ is full.

3.2.2(c) Numerical construction of the hydrodynamical matrices. Two cases are considered.

General case: The surface $\Sigma$ is any surface of $\mathbb{R}^{3}$ and there may be a free surface. The hydrodynamical coupling and radiating matrices are built by solving the problem (62) with an exact integral equation formulation on $\Sigma$, which has been devel- oped at ONERA. This method has been developed and validated in a low frequency range $[49,57,24,76]$ and has been improved for the MF range. This last theory is exposed and validated in Ref. [2]. For any $\omega$ in $\mathbb{R}$, no irregular frequencies are obtained. Some general programs have been developed on scalar and vectorial computers. The program FSMFM builds $\mathbf{B}_{H}^{\prime}(\omega)$ and FSMFR builds $\mathbf{R}_{H}^{\prime}(\omega, x)$. For this MF dynamic analysis with fluid-structure interaction, three steps are used. In a first step FSMFM computes $\mathbf{B}_{H}^{\prime}(\omega)$. In a second step ADINA realizes the MF analysis of the coupled problem. In a third step FSMFR calculates the radiated acoustic field. The corresponding programs for an axisymmetric coupled problem are FSMFMA and FSMFRA.

Particular case: The surface $\Sigma$ is a slender axisymmetric surface and there is no free surface (the coupled problem is not necessarily axisymmetric). In this case the coupling and radiating matrices are built by using a hydrodynamical assymptotic theory developed at ONERA for the slender surface. This theory has been developed and validated in low frequency[20,86] and has been modified and validated for the MF range[88]. The gain obtained on the numerical costs is large enough, but this method can be employed only for particular cases. The programs FSMFME and FSMFRE have been developed for $3 D$ case, i.e. for an unaxisymmetric coupled problem. The corresponding programs for an axisymmetric coupled problem are FSMFEA and FSMFREA.

3.2.2(d) To obtain the MF model equation. To obtain the MF model equation (30), starting from eqn (59), we use the method exposed in Sec. 2.4. We note that the functions $\omega \rightarrow \mathbf{M}(\omega)=\mathbf{M}_{S}+\mathbf{M}_{H}(\omega)$ and $\omega \rightarrow \mathbf{C}(\omega)=\mathbf{C}_{S}+\mathbf{C}_{H}(\omega)$ verify the hypotheses of Sec. 2.1. Consequently, for a narrow MF band $B_{n}$, we have

$\mathbf{M}_{n}=\mathbf{M}_{S}+\mathbf{M}_{H}\left(\Omega_{n}\right) ; \mathbf{C}_{n}=\mathbf{C}_{S}+\mathbf{C}_{H}\left(\Omega_{n}\right) ; \mathbf{K}_{n}=\mathbf{K}_{S}$.

This approximation, which is described in Sec. 2.4, is very efficient because $\omega \rightarrow \mathbf{M}_{H}(\omega)$ and $\omega \rightarrow \mathbf{C}_{H}(\omega)$ are smooth functions due to the fact that the fluid domain is unbounded [no spectral problem is associated to the Neumann problem (62)]. We have studied in details the validity of this approximation in Refs [89 and 90].

If $\mathbf{U}_{n}$ is the MF solution of the coupled problem for the MF narrow band $\mathbf{B}_{n}$, which is built with the general method exposed in part I, the radiated acoustic field is written as

$$
\begin{aligned}
\hat{p}(\omega, x) & =-\left.\omega^{2} \mathbf{R}_{H}^{\prime}\left(\Omega_{n}, x\right) \hat{\mathbf{U}}_{n}(\omega)\right|_{\Sigma} \\
& =\left.\mathbf{R}_{H}^{\prime}\left(\Omega_{n}, x\right) \hat{\mathbf{U}}_{n}(\omega)\right|_{\Sigma},
\end{aligned}
$$

where $x \in L,\left.\mathbf{U}_{n}\right|_{\Sigma}$ is the restriction to $\Sigma$ of $\mathbf{U}_{n}$, and where $\ddot{\mathbf{U}}_{n}(\omega)$ is calculated with the formula given in Sec. 2.5.2. 
3.2.3 Corresponding development in ADINA. For the linear MF dynamic analysis we have implanted in ADINA a special fluid element. The input data of this element concerns the list of the numbers of the fluid nodes of $\Sigma$ and the fluid DOFs. During the assembling phase, the $\left(m^{\prime} \times m^{\prime}\right)$ full complex symmetric matrix $\mathbf{B}_{H}^{\prime}\left(\Omega_{n}\right)$ is read on a file which has been generated by step (1). At the end of step (2) with ADINA, a file containing $\left.\mathbf{W}_{0}\left(l_{\mathrm{L}}\right)\right|_{\Sigma}$, $l \in\left[-\mathbf{L}_{l}, \mathbf{L}_{F}\right]$ is generated [see eqn (46)]. This file is read in step (3) to calculate the radiated acoustic field [eqn (74)].
3.2.4 Validation. The proposed MF method and the programs have been validated by comparing the results given (1) with some analytical solutions (e.g. an elastic sphere[2]), (2) with the numerical solutions computed with other validated programs developed at ONERA for LF domain (e.g. an elastic circular cylinder with bottoms)[88-90] (note that the MF method can be used in LF range in order to validate the MF programs), and (3) with MF experimental measures realized by ONERA on several systems[58, 91]. We present hereafter an example with experimental comparisons taken in Ref. [26].

(a)

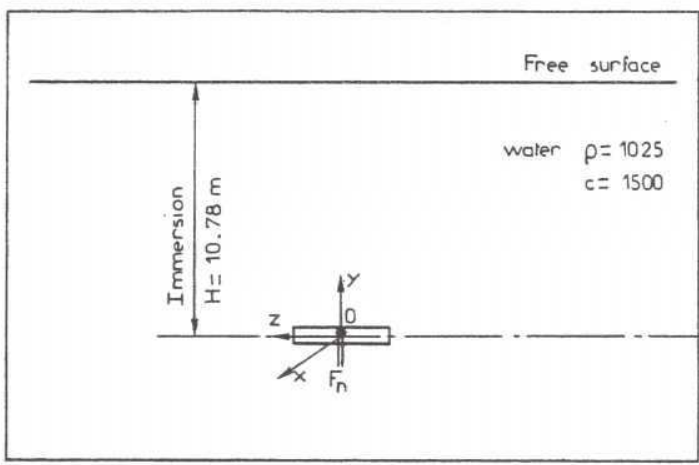

(b)

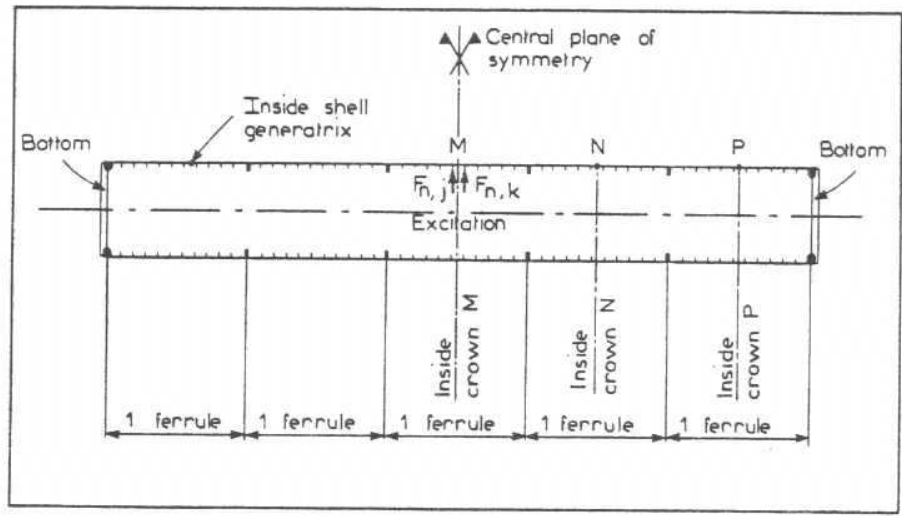

(c)

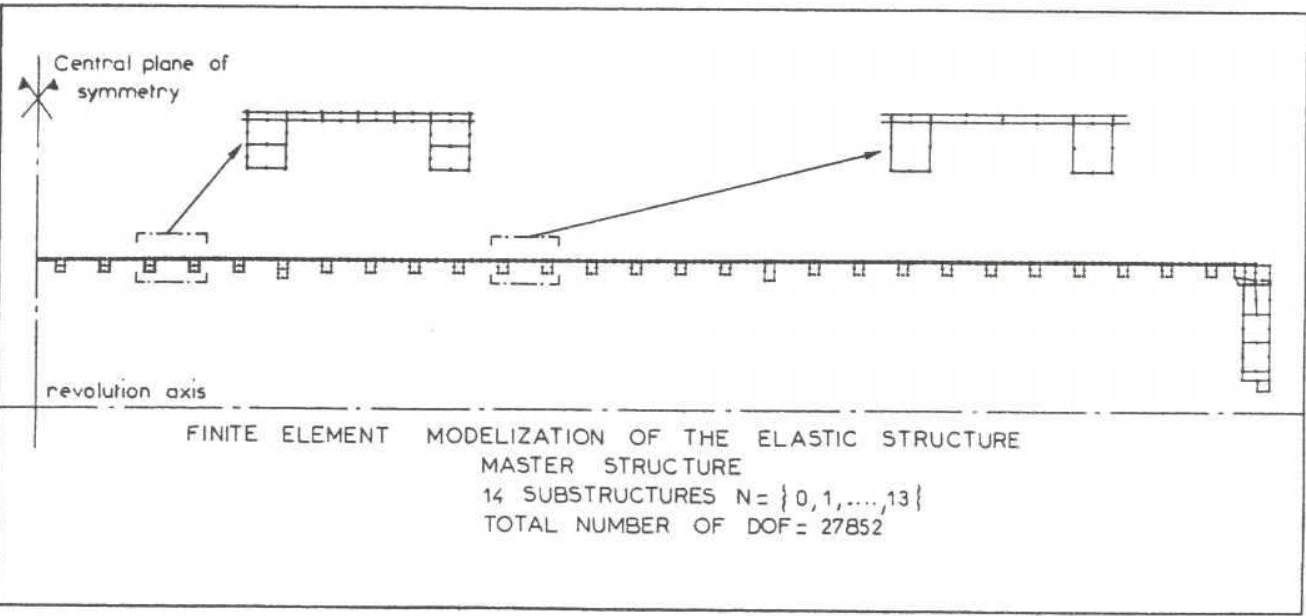

Fig. 8. Three-dimensional stiffened shell cylinder immersed in an unbounded compressible fluid. (a) Geometry and fluid properties; (b) excitation force and observation points; (c) finite element modelization of the elastic structure. (Continued on following page.) 


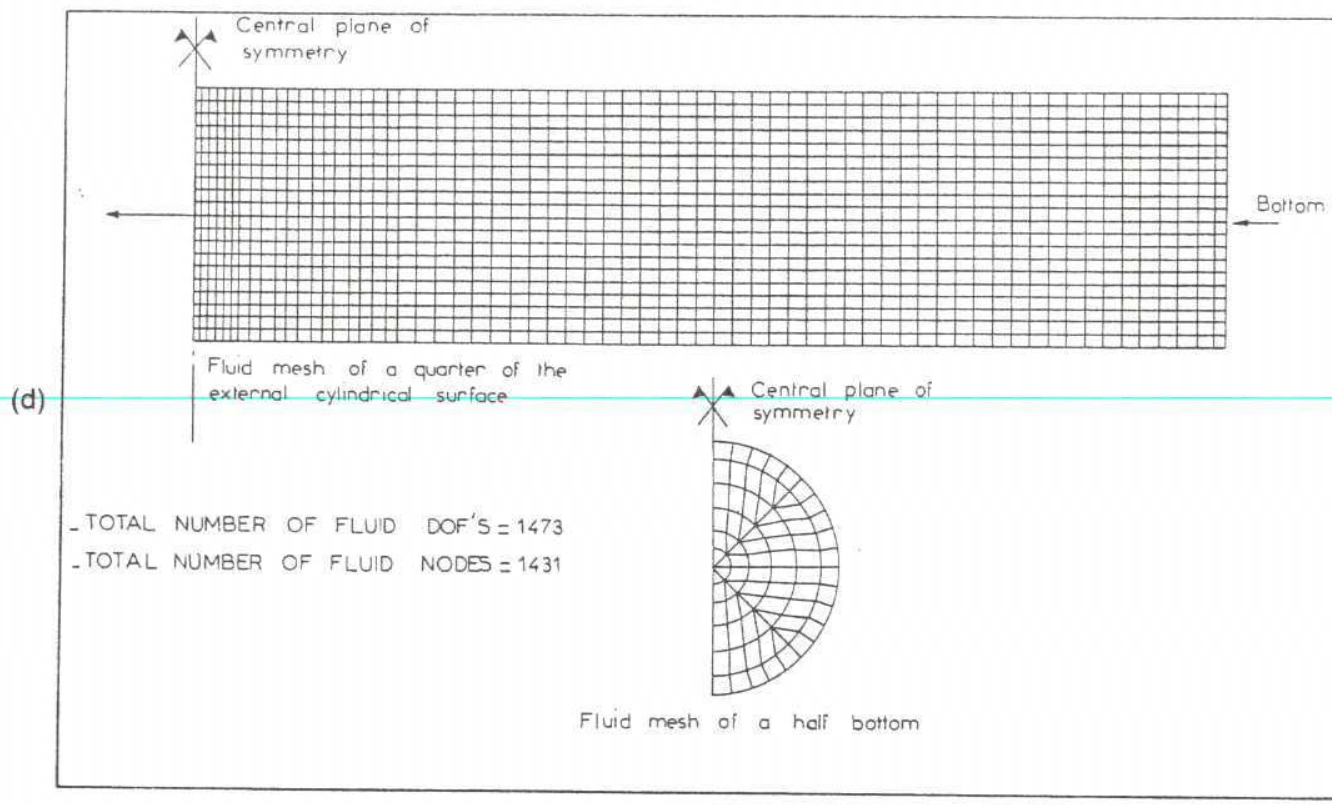

Fig. 8(d). Fluid mesh of the fluid-structure interface.

3.2.5 Hydro-elasto-acoustic of a stiffened shell cylinder. In this section we give an example of a MF hydro-elasto-acoustic calculation with experimental comparisons. We consider the structure defined at Sec. 3.1.5, but the bottoms are closed and there is no floor. Thus the geometry and the material properties are defined by Fig. 2(a). This structure is immersed in an unbounded compressible fluid (water) and there is a free surface. The fluid properties and immersion depth are given in Fig. 8. This structure has axisymmetric part but is not axisymmetric due to the internal subsystems. On the other hand, the fluid-structure coupled problem is not axisymmetric because there is a free surface. The MF linear dynamic calculations of the coupled system are realized for the broad frequency band $B=[2000-4000] \mathrm{Hz}$. This band is partitioned into $20 \mathrm{MF}$ narrow bands $B_{n}$ of bandwidth $100 \mathrm{~Hz}$. For each band $B_{n}$, we apply two excitation forces $\mathbf{F}_{n, j}$ and $\mathbf{F}_{n, k}$ which are defined in Sec. 2.3.1(b). The spatial parts $\mathbf{A}_{j}$ and $\mathbf{A}_{k}$ are two concentrated forces located symmetrically with regard to the central plane of symmetry [see Fig. 8(b)]. The modelization of the elastic structure is realized with the method described in Sec. 3.1.2(b) and is meshed with axisymmetric $2 \mathrm{D}$ solid elements. We have considered 14 values of $N$ (circumferential node pattern): $N \in\{0$, $1,2, \ldots, 13\}$, with an evolutive mesh. Taking into account two planes of symmetry, only a quarter of the structure is considered [see Fig. 8(c)]. The total number of structural DOFs is 27852 . The mass matrix is consistent and the structural damping matrix is generated by eqn (57). The mesh of the interface is defined in Fig. 8(d). There is $m^{\prime}=1473$ fluid DOFs for a quarter of the $\Sigma$ interface. A free surface is taken into account. For each $B_{n}$ band the hydrodynamical matrices $\mathbf{B}_{H}^{\prime}\left(\Omega_{n}\right)$ and $\mathbf{R}_{H}^{\prime}\left(\Omega_{n}, x\right)$ are computed with the general method and with the programs FSMFM and FSMFR. The calculations have been made with a CRAY IS/2000 computer.

We give a few numerical results of the complete analysis which has been made on this coupled system[26] and some experimental results realized by ONERA[58].

Figure 9(a) shows the evolution with the frequency bands $B_{n}$ of $10 \log _{10} E$, where $E=E_{u l}\left(B_{n}\right)$ is calculated with eqn $(50)$, at two observation points of the elastic structure. Experimental comparisons are made. Figure $9(b)$ shows the graph of $j, n \rightarrow 10 \log _{10} E_{i j}\left(B_{n, j}\right)$ for a given observation point on the shell, close to the excitation force. Figures $9(\mathrm{c})$ and $9(\mathrm{~d})$ show the spatial propagation of the normalized energy $\bar{E}=E_{4}\left(B_{n}\right) / E_{\max }$ of the radial acceleration $\ddot{q}$ for the $B_{n}$ bands [2700-2800] and [3600-3700] Hz. Experimental comparisons are given. Figures 10(a) to 10(d) are related to the energy $E_{p}\left(B_{n}\right)$ of the radiated acoustic field $p$ for the band $B_{n}=[3600-3700] \mathrm{Hz}$. Figure 10(a) shows the radiation diagram with a free surface. Figures $10(b)$, 10 (c), and 10(d) show the radiation diagram without free surface.

\subsection{Linear MF vibrations of elastic structures coupled} with an internal viscous compressible bounded fluid

3.3.1 Setting the problem. In this section we study the linear fluid-structure interactions in the MF range of a 3D linear elastic body with a viscous compressible fluid which occupies a bounded domain. For example, let us consider a steel or aluminium elastic structure with a large enough internal cavity filled with a light fluid (e.g. air). When we study such a coupled problem in a broad frequency band, we can obtain an MF behavior for the structure and an LF behavior for the internal fluid 


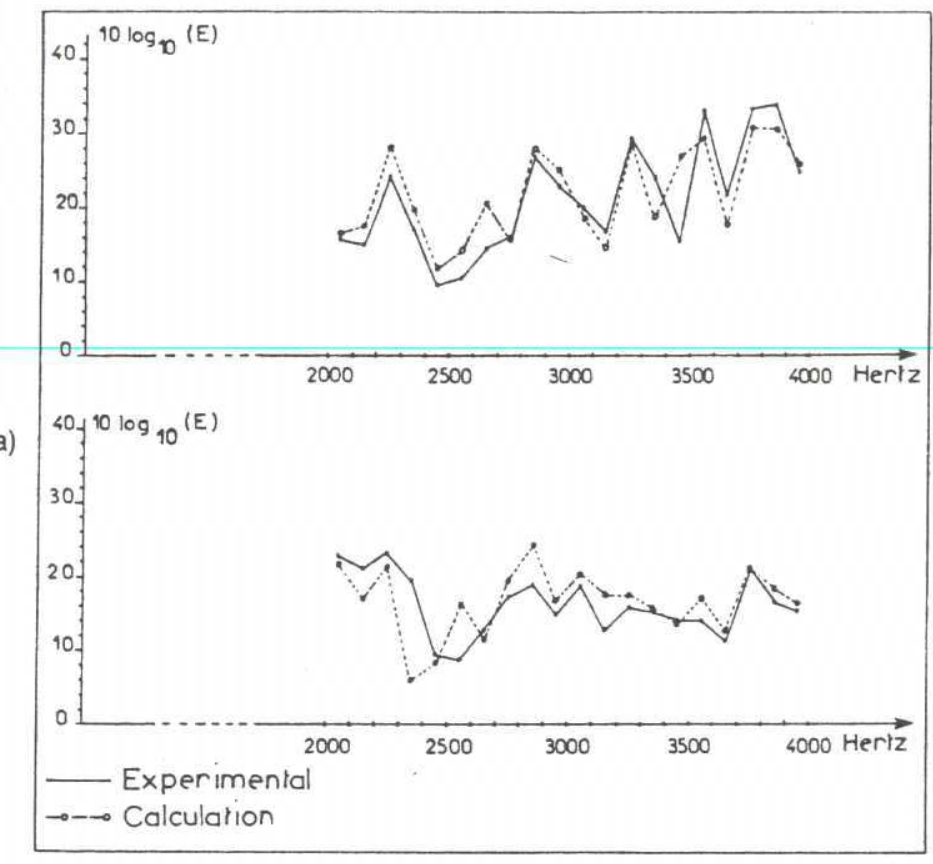

(b)

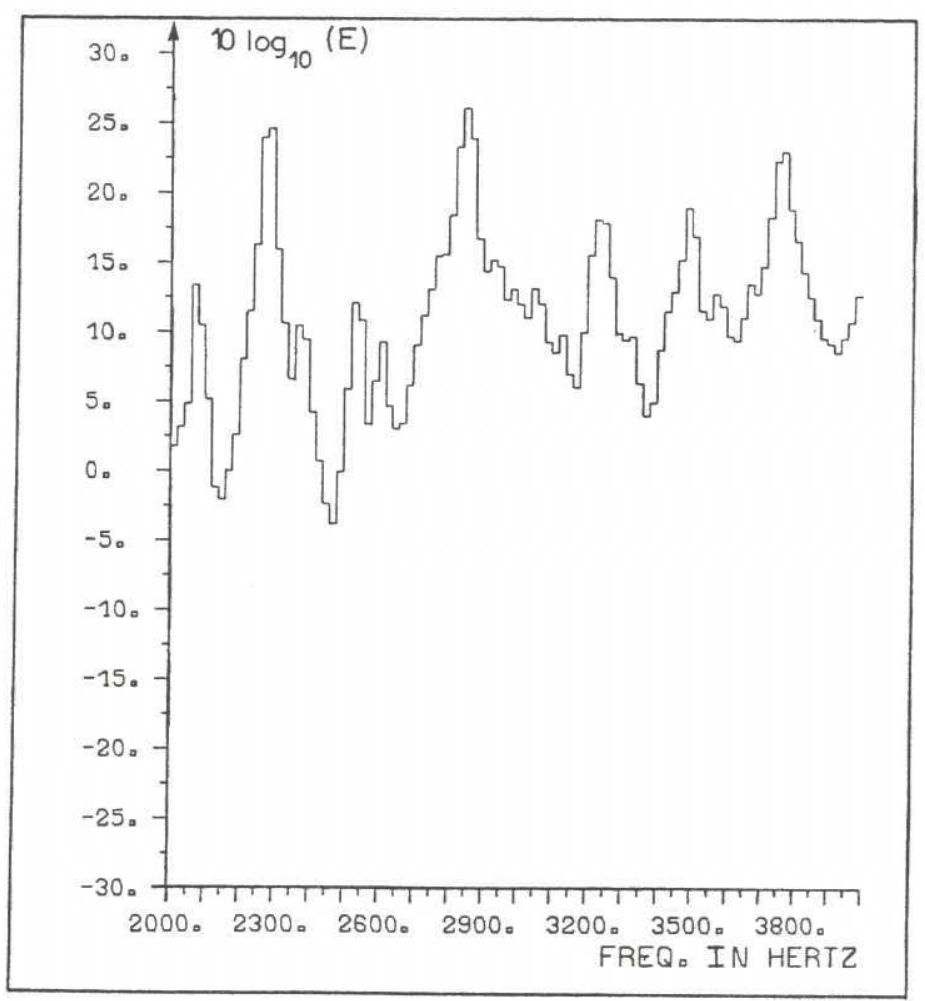

Fig. 9. Three-dimensional stiffened shell cylinder immersed in an unbounded compressible fluid with a free surface. Experimental comparisons. (a) Evolution of the radial acceleration energy per band at two observation points of the elastic structure coupled with the fluid. Experimental comparisons. (b) Frequency response function expressed in terms of energy. $E=$ energy of the radial acceleration integrated by frequency band of $20 \mathrm{~Hz}$ at an observation point on the shell. (c) Spatial propagation of the normalized energy of the radial acceleration in the band [2700-2800] Hz. (d) Spatial propagation of the normalized energy of the radial acceleration in the band [3600-3700] Hz. ((c) and (d) appear on the following page.) 

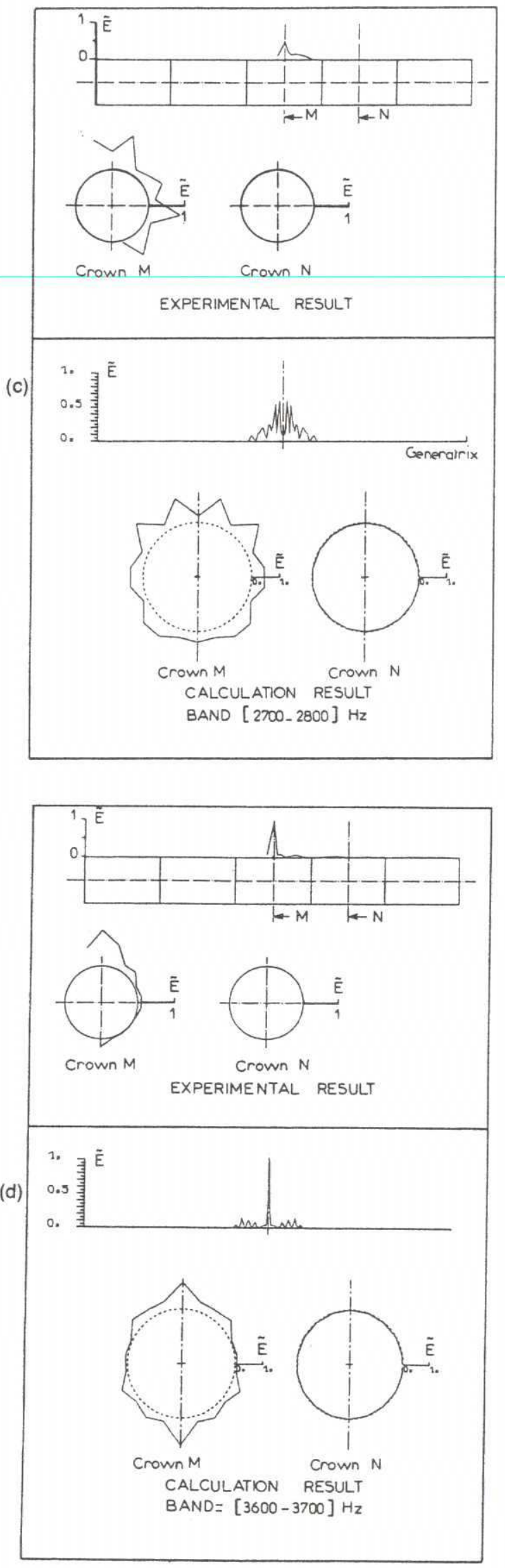

Fig. 9(c), (d). Captions appear on the previous page.

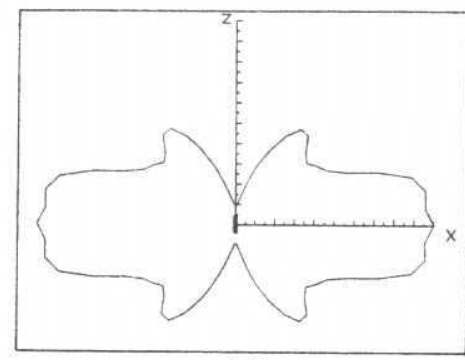

(a)

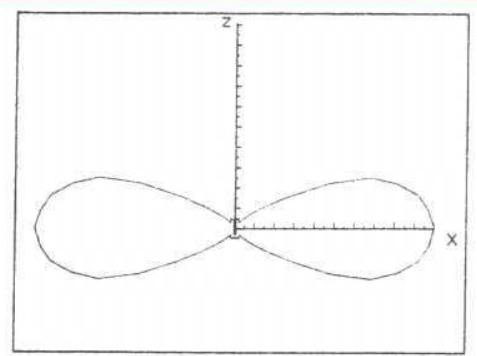

(b)

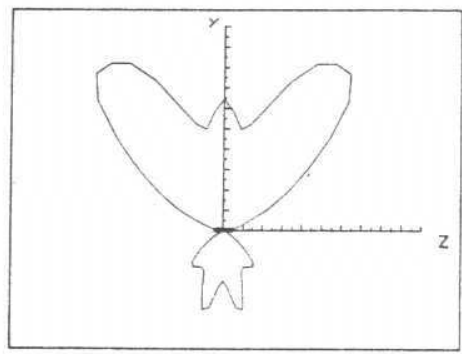

(c)

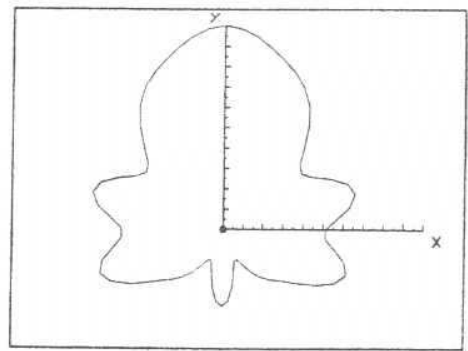

(d)

Fig. 10. Radiation diagram. Energy in the band [3600$3700] \mathrm{Hz}$ of the radiated acoustic field by a 3D stiffened shell cylinder immersed in an unbounded compressible fluid with and without free surface. (a) With free surface. Distance from the origine $70 \mathrm{~m}$. Plane parallel at $\mathrm{XOZ}$. Immersion $11.5 \mathrm{~m}$. (b) Without free surface. Distance from the origine $70 \mathrm{~m}$. Plane parallel at $\mathrm{XOZ}$. Immersion 11.5 m. (c) Without free surface. Distance from the origine 70 $\mathrm{m}$. Plane YOZ. (d) Without free surface. Distance from the origine $70 \mathrm{~m}$. Plane $X O Y$.

(i.e. the first acoustical eigenmodes of the internal cavity with a perfectly rigid boundary, have eigenfrequencies in the analyzed frequency band). Consequently, we must use a fluid formulation which allows us to take into account this situation. It is classically obtained if the internal fluid is governed by an interior Neumann problem associated with the Helmholtz equation, i.e. the fluid is perfect and compressible. On the other hand, if the cavity has 
a rigid boundary, we want the level pressure to be controlled in the cavity when an excitation source is applied into the fluid. Thus, we must introduce the viscosity of the fluid.

For the fluid, one can use either an integral formulation on the interface $\Sigma$, or a finite element formulation in the bounded interior domain. The first formulation introduces a coupling fluid operator $\omega$ $\rightarrow D(\omega)$ which depends on frequency. Thus, the hypotheses introduced at the end of Sec. 2.4 are not satisfied because $\omega \rightarrow D(\omega)$ is not a smooth function, due to the existence of the associated interior spectral problem. Consequently we shall use the finite element formulation in the cavity. In this case the mass, damping and stiffness matrices of the fluid do not depend on frequency, and the general MF method developed in part I is applicable.

With the proposed MF method, we do not need to solve the eigenvalue problem related to the associated conservative coupled problem. Consequently we shall use for this fluid-structure interaction problem a symmetric formulation with only one unknown scalar functional to describe the fluid state. Thus a node of a fluid finite element has only one DOF. The fluid-structure coupling matrix is classically generated with fluid-structure interface finite elements, and is assembled in the global damping matrix. So, we obtain a consistent damping matrix for the coupled problem. Note that we have already introduced a consistent damping matrix in the MF method (see Sec. 2.6).

3.3.2 Theoretical complements. We consider an elastic body which occupies an open bounded domain $S$ of $\mathbb{R}^{3}$ with smooth boundary $\partial S=\Sigma_{1} \cup \Sigma$ and an internal fluid which occupies an open bounded domain $L$ such that $\partial L=\Sigma$. Let $x$ be a generating point of $\mathbb{P}^{3}$ and $\alpha$ be the unit normal to $\partial S$ external to the domain $S$. We let $\alpha^{\prime}=-\alpha$ (see Fig. 11). We study the linear vibrations of the coupled system around a static equilibrium state. The hypotheses on the elastic structure are those of Sec. 3.1, and we use same method for its modelization. The mass, damping and stiffness matrices $\left(\mathbf{M}_{S}, \mathbf{C}_{S}\right.$, and $\mathbf{K}_{S}$ ) of the discretized elastic structure verify the hypotheses of part I. The fluid is compressible and viscous, and we assume that the entropy is a constant. We denote by $\rho, c$, and $\eta$ the mass den-

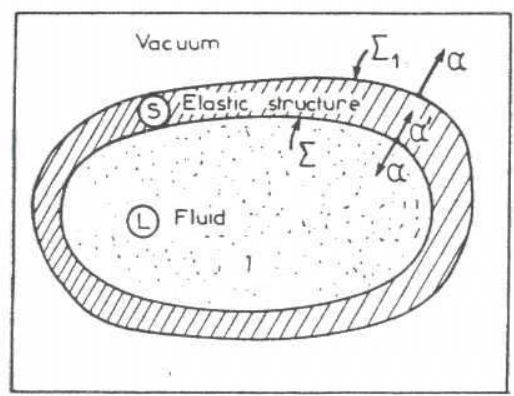

Fig. 11. sity, the speed of sound and the dynamic viscosity coefficient of the fluid in its equilibrium state. We assume $\rho, c$, and $\eta$ to be constant. The kinematic viscosity coefficient is $v=\eta / \rho$.

3.3.2(a) Fluid equations. Let $V=\left(V_{1}, V_{2}, V_{3}\right)$ be the fluid velocity and $p$ be the pressure field fluctuation around the equilibrium state. We suppose for the moment that there is no excitation source into $L$. In these conditions, the continuity equation is

$$
\frac{1}{c^{2}} \frac{\partial p}{\partial t}+\rho \operatorname{div} V=0
$$

and the linearized equation of movements is

$$
\rho \frac{\partial V}{\partial t}=-\operatorname{grad} p+\eta\left(\Delta V+\frac{1}{3} \operatorname{grad} \operatorname{div} V\right),
$$

where $\Delta=\operatorname{div}$ grad is the Laplacian operator in $\mathbb{R}^{3}$. Using the formulas div rot $V=0$, rot rot $V=$ grad div $V-\Delta V$, applying the div operator to the two members of eqn (76), and considering eqn (75), we obtain

$$
\frac{1}{c^{2}} \frac{\partial^{2} p}{\partial t^{2}}-\frac{4}{3} \frac{\eta}{\rho c^{2}} \Delta \frac{\partial p}{\partial t}-\Delta p=0 .
$$

We are searching for a solution in the form

$$
p=-\rho \frac{\partial \phi}{\partial t} \text { into } \bar{L}=L \cup \Sigma
$$

where $\phi$ is the new unknown functional. Note that we have $V \neq \operatorname{grad} \phi$, because rot $V$ is not null into $L$. Substituting eqn (78) into eqn (77) we obtain

$$
\frac{1}{c^{2}} \frac{\partial^{2} \phi}{\partial t^{2}}-\left(1+\frac{4}{3} \frac{\eta}{\rho c^{2}} \frac{\partial}{\partial t}\right) \Delta \phi=0 \text { into } L \text {. }
$$

As we explained in the preceding paragraph, we write rot $V \sim 0$ in a neighborhood of $\Sigma$. Thus, using eqns (75) and (78), eqn (76) gives

$$
V \sim\left(1+\frac{4}{3} \frac{\eta}{\rho c^{2}} \frac{\partial}{\partial t}\right) \operatorname{grad} \phi=0 \text { on } \Sigma .
$$

Let $u=\left(u_{1}, u_{2}, u_{3}\right)$ be the elastic structure displacement field. Consequently, the boundary condition on $\Sigma$, for the fluid, is written as

$$
\left(1+\frac{4}{3} \frac{\eta}{\rho c^{2}} \frac{\partial}{\partial t}\right) \frac{\partial \phi}{\partial \alpha^{\prime}}=\left\langle\frac{\partial u}{\partial t}, \alpha^{\prime}\right\rangle_{3} \text { on } \Sigma
$$

and the boundary condition on $\Sigma$, for the elastic structure, is

$$
\sigma(\alpha)=-p \alpha \text { on } \Sigma,
$$

where $\sigma$ is the 3D stress tensor of the elastic body. 
3.3.2(b) Variational formulation for the fluid and finite elements. Let $\varphi$ be a test function belonging to an admissible class of functions. Using Green's formula, and since $\alpha^{\prime}$ is the exterior normal to the fluid, we have

$$
\begin{aligned}
{\left[2 \frac{\partial^{2} \phi}{\partial t^{2}}, \varphi\right] } & +\frac{4}{3} \frac{\eta}{\rho c^{2}}\left[\mathscr{R} \frac{\partial \phi}{\partial t}, \varphi\right] \\
& +[\Re \phi, \varphi]-\left[\left(\mathscr{H} \varphi, \frac{\partial u}{\partial t}\right)\right]=0
\end{aligned}
$$

where the linear operators $\mathscr{2}, \mathscr{R}$, and $\mathscr{H}$ are defined by

$$
\begin{aligned}
{[2 \phi, \varphi] } & =\frac{\rho}{c^{2}} \int_{L} \phi \varphi \mathrm{d} x, \\
{[\mathscr{R} \phi, \varphi] } & =\rho \int_{L}\langle\operatorname{grad} \phi, \operatorname{grad} \varphi\rangle_{3} \mathrm{~d} x, \\
{[(\mathscr{H} \phi, u)] } & =\rho \int_{\Sigma} \varphi\left\langle u, \alpha^{\prime}\right\rangle_{3} \mathrm{~d} \Sigma .
\end{aligned}
$$

The fluid-structure coupling operator, denoted by $\mathscr{C}_{L S}$, is defined by the following bilinear form:

$$
\left(\begin{array}{l}
u \\
\phi
\end{array}\right),\left(\begin{array}{l}
v \\
\varphi
\end{array}\right) \rightarrow[(\mathscr{H} \phi, v)]+[(\mathscr{H} \varphi, u)] .
$$

Using the finite element formulation, we obtain classically the matrices $\mathbf{Q}, \mathbf{R}$ and $\mathbf{C}_{L S}$ of the operators $2, \mathscr{R}$, and $\mathscr{C}_{L S}$, where the matrix $\mathbf{C}_{L S}$ is written as

$$
\mathbf{C}_{L S}=\left[\begin{array}{cc}
0 & \mathbf{H} \\
\mathbf{H}^{T} & 0
\end{array}\right],
$$

and where $\mathbf{H}$ is the matrix of $\mathscr{H}$.

3.3.2(c) Discretized equation of the coupled system. Denoting by $\phi_{n}$ the column matrix of the fluid DOF's, the MF vibration equation of the discretized coupled system is written in the Fourier space as

$$
\left(-\omega^{2} \mathbf{M}_{n}+i \omega \mathbf{C}_{n}+\mathbf{K}_{n}\right)\left(\begin{array}{c}
\hat{\mathbf{U}}_{n} \\
\hat{\phi}_{n}
\end{array}\right)=\left(\begin{array}{c}
\hat{\mathbf{F}}_{n} \\
0
\end{array}\right),
$$

where the matrices $\mathbf{M}_{n}, \mathbf{C}_{n}$, and $\mathbf{K}_{n}$ are such that

$$
\begin{aligned}
& \mathbf{M}_{n}=\left[\begin{array}{cc}
\mathbf{M}_{S} & 0 \\
0 & -Q
\end{array}\right] ; \\
& \mathbf{C}_{n}=\left[\begin{array}{cc}
\mathbf{C}_{S} & \mathbf{H} \\
\mathbf{H}^{T} & -\frac{4}{3} \frac{\eta}{\rho c^{2}} \mathbf{R}
\end{array}\right] ; \\
& \mathbf{K}_{n}=\left[\begin{array}{cc}
\mathbf{K}_{S} & 0 \\
0 & -\mathbf{R}_{j}
\end{array}\right] .
\end{aligned}
$$

If there is an excitation source in the fluid, the null term in the right member of eqn (87) must be replaced by the excitation term. On the other hand, to take into account the loss phenomenas in the neighborhood of $\Sigma$, which are not directly modelized in the present model, one can artificially increase the value of $\eta$ for the fluid finite elements located close to $\Sigma$. Note that the eqn (87) is a MF model equation of the type (30). The matrices $\mathbf{M}_{n}$, $\mathbf{C}_{n}$, and $\mathbf{K}_{n}$ are constant, real and symmetric. We see that the hypotheses introduced in Sec. 2.1, i.e. $\mathbf{M}_{n}, \mathbf{C}_{n}$, and $\mathbf{K}_{n}$, are positive definite matrices, is not systematically verified. But this point is not important. Indeed the necessary hypothesis is the frequency response function $\omega \rightarrow T(\omega)$ [defined by eqn (15)] to be a continuous and bounded mapping on $\mathbb{R}$. So, one can easily verify[27] that this hypothesis is satisfied for eqn (87) if $\mathbf{M}_{n}, \mathbf{C}_{n}$, and $\mathbf{K}_{n}$ verify all the hypotheses of Sec. 2.1.

3.3.3 Corresponding development in ADINA. We have implanted in ADINA:

(a) Compressible viscous fluid finite elements which generate a "mass" matrix - $\mathbf{Q}$, a consistent "damping" matrix $-4 / 3\left(\eta / \rho c^{2}\right) \mathbf{R}$, and a "stiffness" matrix $-\mathbf{R}$. Each element node has one DOF. The elements are: (1) 2D fluid element isoparametric quadrilateral with a number of nodes $4-8$. The element can be used either for a two-dimensional or for an axisymmetric $N=0,1,2 \ldots$ problem. (2) Three-dimensional fluid element isoparametric or subparametric curvilinear hexahedra with a variable number of nodes 8-21.

(b) Fluid-structure interface finite elements which generate a consistent damping matrix $\mathrm{C}_{L S}$ defined by eqn (86). An interface finite element has fluid nodes and structural nodes. At each fluid nodes corresponds a structural node having the same coordinates. The elements are: (1) ID interface element isoparametric curvilinear line with $2 \times 2$ or $2 \times 3$ nodes. The element can be used either for a two-dimensional or an axisymmetric problem. (2) Two-dimensional interface element isoparametric curvilinear quadrilateral with a variable number of nodes $2 \times 4$ to $2 \times 8$.

3.3.4 Validation. The validation has been made by comparing the MF numerical results with known analytical solutions. These comparisons are given in Ref. [27].

3.3.5 Example. We consider a shell circular closed cylinder with elastic bottoms and internal fluid. We are studying the MF vibrations of this system subjected to an axisymmetric loading in the broad frequency band $B=[0-4000] \mathrm{Hz}$. This band is partitioned into $40 \mathrm{MF}$ narrow bands of bandwidth $100 \mathrm{~Hz}$. The geometry, the mechanical properties and the finite element modelization are given in Fig. 12. Taking into account one plane of symmetry, only half-system is modelized. For the elastic structure, the mass matrix is consistent and the structural damping matrix is generated by eqn (57) with $\xi_{n}=0.003$.

3.3.5(a) Influence of the elasticity of the walls. The fluid is excited with axisymmetric ponctual sources applied to all the fluid nodes located on the $O Y$ and 


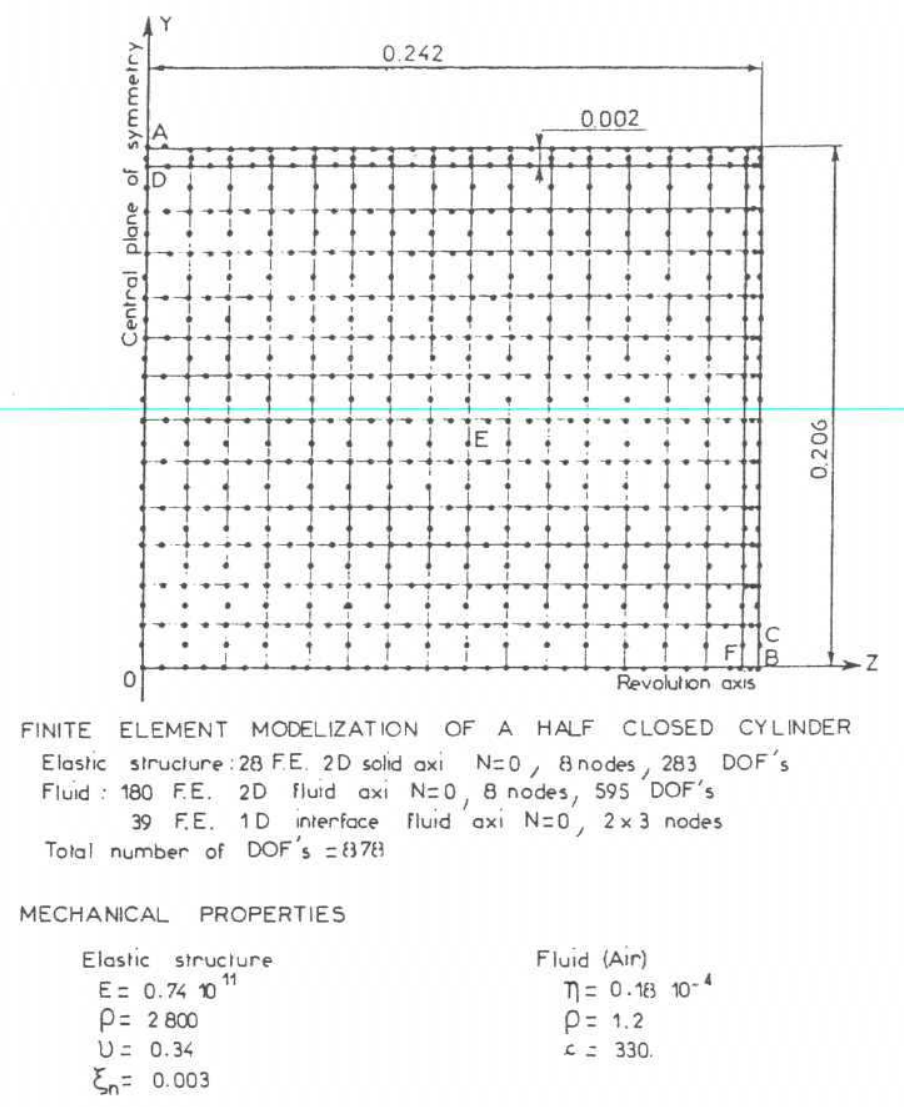

Fig. 12. Fluid-structure interaction with a closed cylinder. Finite element modelization and mechanical properties.

$O Z$ axes (see Fig. 12). The frequential part of the excitation is $\hat{\mathbf{1}}_{B_{n}}(\omega)$ on each band $B_{n}$. Two cases are analyzed: (1) the structure is perfectly rigid with internal fluid, (2) the structure is elastic with internal fluid. Figure 13(a) shows, for the two cases considered, the graphs of $j, n \rightarrow 10 \log _{10} E_{p / p}\left(B_{n, j}\right)$, where $p$ is the pressure at the fluid observation point $O$ (see Fig. 12). This figure shows the influence of the elasticity of the walls on the fluid pressure. Figures 13(b) to 13(e) show, for the two analyzed cases, the spatial repartition in the fluid of the normalized energy $\bar{E}=E_{p / p}\left(B_{n}\right) / E_{\max }$ for bands [1000-1100] and [2600-2700] Hz.

3.3.5(b) Influence of the internal fluid on the structural vibrations. The elastic structure is excited with a radial concentrated force applied to point $A$ (see Fig. 12). The amplitude is 1 and the frequential part is $\hat{\mathbf{1}}_{B_{n}}(\omega)$ on each $B_{n}$ band. Two cases are analyzed: (1) the structure is elastic without internal fluid (vacuum) and (2) the structure is elastic with internal fluid.

Figure 14(a) shows, for the two cases, the graphs of $j, n \rightarrow 10 \log _{10} E_{\dot{q}}\left(B_{n, j}\right)$, where $\dot{q}$ is the normal velocity of the structure to the observation point $B$ (see Fig. 12). This figure shows the influence of the internal fluid on the vibratory behavior of the elastic structure. Figures 14(b) to 14(d) show, for the two analyzed cases, the spatial propagation of the normalized energy $\hat{E}=E_{\dot{q}}\left(B_{n}\right) / E_{\max }$ for the band $B_{n}=$ [1600-1700] Hz.

\subsection{Linear MF vibrations of elastic structures with viscoelastic materials}

3.4.1 Setting the problem. In this section we study the MF linear vibrations of an elastic structure with viscoelastic materials. The viscoelasticity introduces a temporal memory. Consequently in the Fourier domain, the MF vibrations are governed by an equation of type (29). So, we shall use the method described in Sec. 2.4 to obtain the model MF equation (30).

3.4.2 Theoretical complements. Let us consider an isotropic linear viscoelastic material. Let $u(x t)$ be a 3D displacement field, where $x=\left(x_{1}, x_{2}, x_{3}\right)$ is a generating point $\mathbb{R}^{3}$. Let $\sigma_{j k}$ and $\epsilon_{j k}$ be, respectively, the $3 \mathrm{D}$ stress and strain tensors. We have $\epsilon_{j k}(u)=1 / 2\left(\partial u_{j} / \partial x_{k}+\partial u_{k} / \partial x_{j}\right)$. In the classical theory of linear viscoelasticity[11, 12, 42] the stressstrain relation in the Fourier domain is written as

$\hat{\sigma}_{j k}(\omega, \hat{u})=\lambda(\omega) \delta_{j k} \Sigma_{l=1}^{3} \epsilon_{\rho \rho}(\hat{u})+2 \mu(\omega) \epsilon_{j k}(\hat{u})$,

where $\mu(\omega)=\mu_{1}(\omega)+i \mu_{2}(\omega)=|\mu(\omega)|[1+i$ tg $\delta_{\mu}(\omega)$ ] is the complex shear modulus, and where 
$\lambda(\omega)$ is the complex number such that

$$
\lambda(\omega)=\mu(\omega) \frac{E(\omega)-2 \mu(\omega)}{3 \mu(\omega)-E(\omega)} .
$$

We have denoted by $E(\omega)=E_{1}(\omega)+i E_{2}(\omega)=$ $|E(\omega)|\left[1+i \operatorname{tg} \delta_{E}(\omega)\right]$ the complex Young's modulus and by $\delta_{\mu}(\omega)$ and $\delta_{E}(\omega)$ the loss factors.

Consequently, in the Fourier domain, a finite element with a linear viscoelastic material has con- sistent damping and stiffness matrices which depend on frequency. The mass matrix is constant. Thus, the MF vibrations equation of the discretized elastic structure with viscoelastic materials is written as

$$
\begin{aligned}
\left\{-\omega^{2}\left(\mathbf{M}_{S}+\right.\right. & \left.\mathbf{M}_{v}\right)+i \omega\left[\mathbf{C}_{S}+\mathbf{C}_{v}(\omega)\right] \\
& +\left(\mathbf{K}_{S}+\mathbf{K}_{v}(\omega)\right\} \hat{\sim}_{n}(\omega)=\hat{\mathbf{F}}_{n}(\omega),
\end{aligned}
$$

where $\mathbf{M}_{S}, \mathbf{C}_{S}, \mathbf{K}_{S}$ and $\mathbf{M}_{v}, \mathbf{C}_{v}, \mathbf{K}_{v}$ are, respectively,

(a)

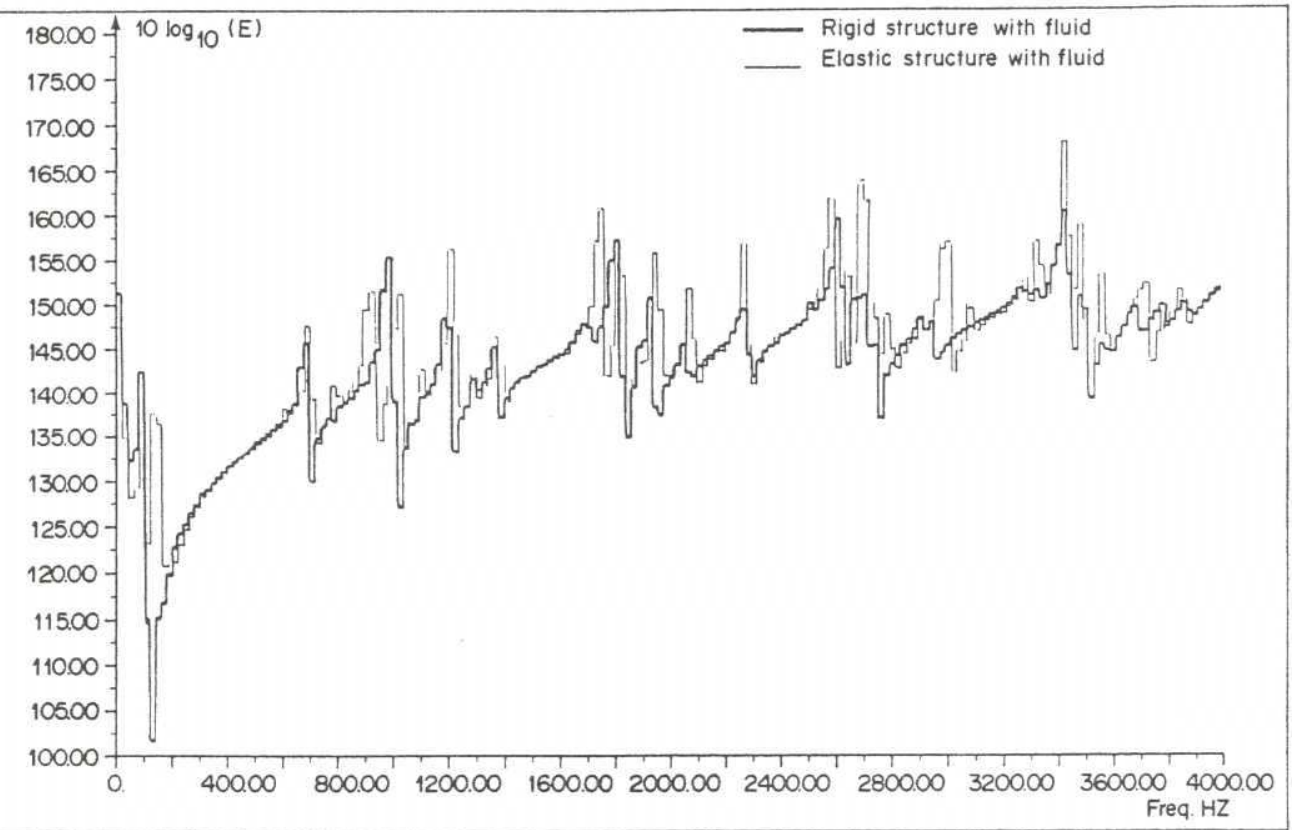

(b)

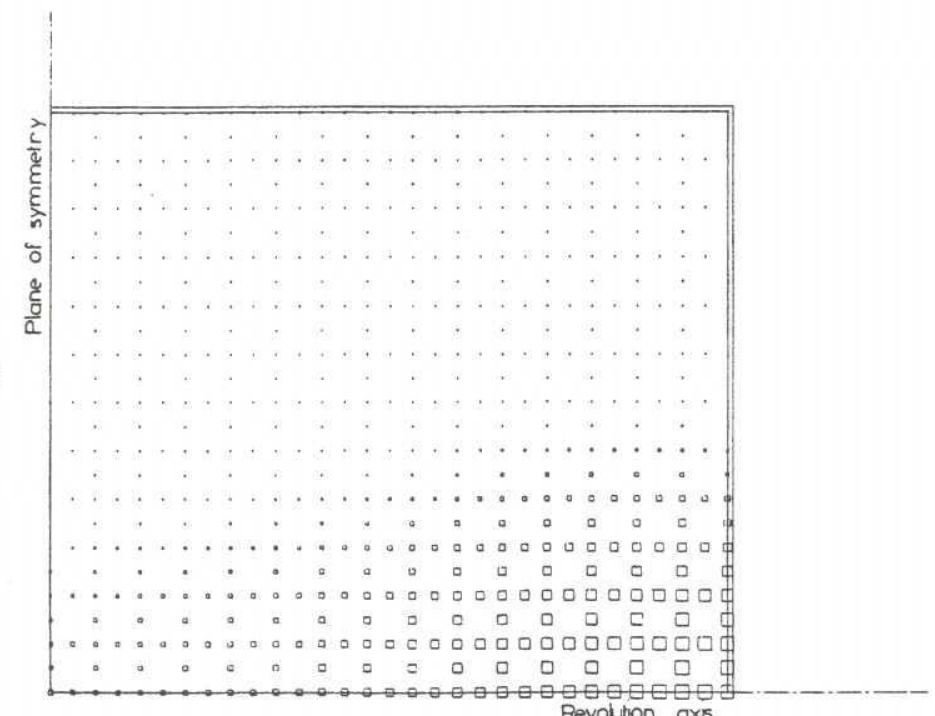

Fig. 13. Influence of the elasticity of the walls of a closed cylinder on the fluid pressure. (a) Frequency response function expressed in terms of energy. $E=$ energy of $p / \rho$ integrated by frequency band of $20 \mathrm{~Hz}$ at the point $O$ into the fluid. (b) Spatial repartition in the fluid of the energy of $p / \rho$ in the band $[1000-1100] \mathrm{Hz}$ with elastic walls. (c) Spatial repartition in the fluid of the energy of $p / p$ in the band $[1000-1100] \mathrm{Hz}$ with rigid walls. (d) Spatial repartition in the fluid of the energy of $p / \rho$ in the band [2600-2700] Hz with elastic walls. (e) Spatial repartition in the fluid of the energy of $p / \rho$ in the band [2600-2700] Hz with rigid walls. ((c)-(e) appear on the following page.) 
(c)

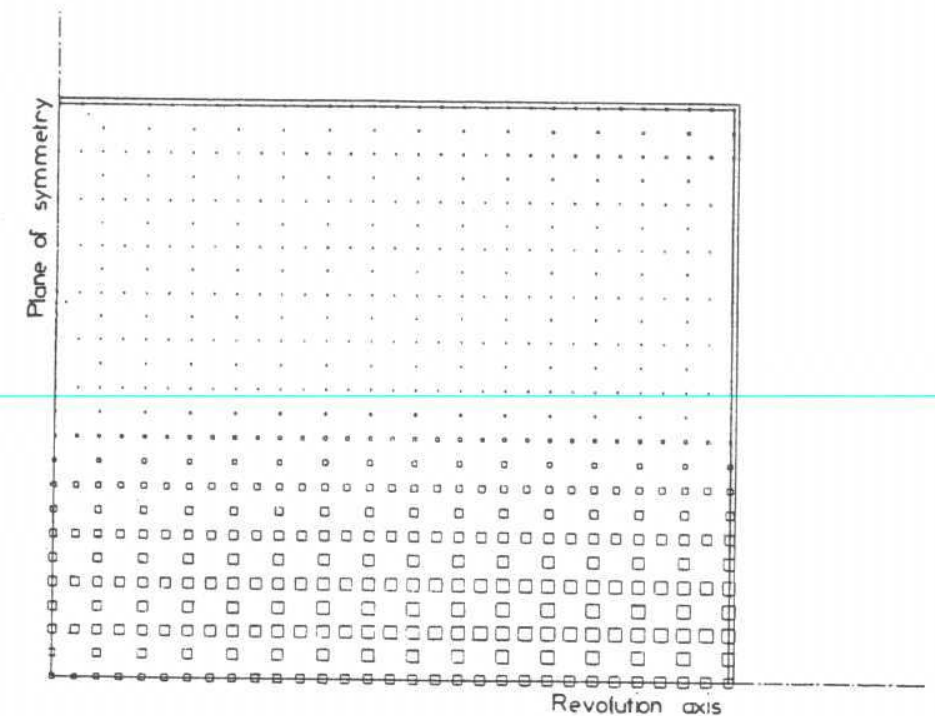

(d)

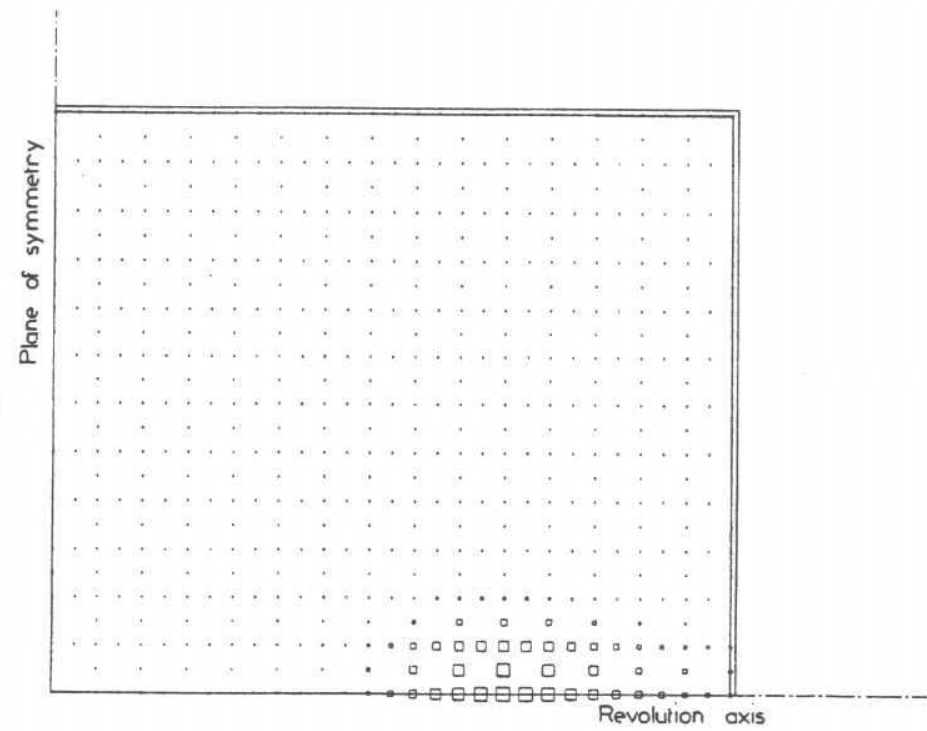

(e)

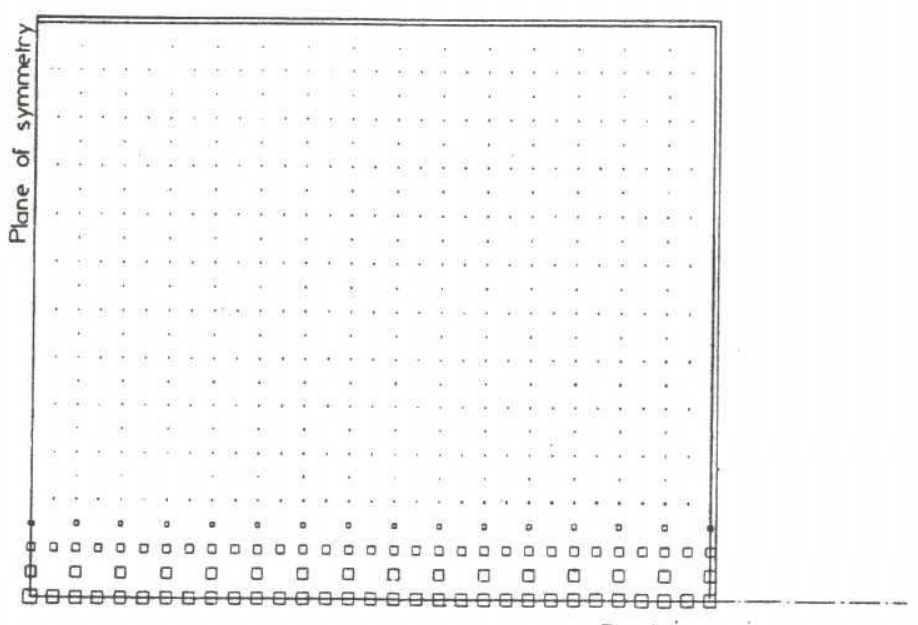

Revolution axis

Fig. 13(c)-(e). Captions appear on the previous page. 
the matrices of the elastic part and of the viscoelastic part. To obtain the MF model equation (30), starting from eqn (91), we use the method exposed in Sec. 2.4. Consequently for a narrow MF band $B_{n}$, we have

$$
\begin{aligned}
\mathbf{M}_{n} & =\mathbf{M}_{S}+\mathbf{M}_{v} ; \\
\mathbf{C}_{n} & =\mathbf{C}_{S}+\mathbf{C}_{v}\left(\Omega_{n}\right) ; \\
\mathbf{K}_{n} & =\mathbf{K}_{S}+\mathbf{K}_{v}\left(\Omega_{n}\right) .
\end{aligned}
$$

We see that the memory of the viscoelastic material is taken into account by writing $B=\cup_{n} B_{n}$, where the bandwidth of each $B_{n}$ is determined in studying the variations of the complex valued functions $\omega \rightarrow$ $E(\omega)$ and $\omega \rightarrow \mu(\omega)$.

3.4.3 Corresponding development in ADINA. For the linear MF dynamic analysis, we have implanted the isotropic linear viscoelastic material model in the 2D and 3D solid elements. For 2D elements, the $N \geqslant 1$ axisymmetric type can be used. These finite elements generate a mass, a consistent damping and a stiffness matrix. For a fixed $B_{n}$ band the material model is defined by the four mechanical constants $\mu_{1}\left(\Omega_{n}\right), \mu_{2}\left(\Omega_{n}\right), E_{1}\left(\Omega_{n}\right)$, and $E_{2}\left(\Omega_{n}\right)$.

3.4.4 Validation. The validation of the new finite elements is made in Ref. [12].

3.4.5 Example. The elastic structure is a steel circular shell cylinder with 11 inside transverse stif- feners. This structure is one ferrule of the system described in Figs. 2(b) and 2(c). An industrial viscoelastic material is placed on the shell between the stiffeners. We have analyzed this system on the frequency band $B=[2000-4000] \mathrm{Hz}$ which is partitioned into $20 \mathrm{MF}$ narrow bands $B_{n}$ of bandwidth $100 \mathrm{~Hz}$. The modelization of this system is realized with the method described in Sec. 3.1.2(b). We have used axisymmetric $2 \mathrm{D}$ solid elements and assembled 11 circumferential node patterns: $N \in\{0$ $1,2, \ldots, 10\}$. The total number of DOF is 12913 . Taking into account the central plane of symmetry, only half of the system is considered. The mechanical properties and the finite element modelization are given in Fig. 15. The functions $\omega \rightarrow|\mu(\omega)|, t g$ $\delta_{\mu}(\omega),|E(\omega)|$, and $\operatorname{tg} \delta_{E}(\omega)$ of the viscoelastic material are defined in Figs 16(a) and 16(b). The elastic structure is excited with a concentrated force (see Fig. 15) with an amplitude of 0.5 and a frequential part $\hat{\mathbf{1}}_{B_{n}}(\omega)$ on each $B_{n}$ band. Two cases are analyzed: (1) elastic structure without viscoelastic material and (2) elastic structure with viscoelastic material.

Figure 17 shows, for the two cases analyzed, the graphs of $j, n \rightarrow 10 \log _{10} E_{\ddot{q}}\left(B_{n, j}\right)$ where $\ddot{q}$ is the radial acceleration of the elastic structure at the excitation point. Figures 18(a) and 18(b) show, for the two considered cases, the spatial propagation of the normalized energy $\hat{E}=E_{\vec{q}}\left(B_{n}\right) / E_{\max }$ into the genaratrix and into the excitation crown for the $B_{n}$ bands [2200-2300] and [3100-3200] Hz.

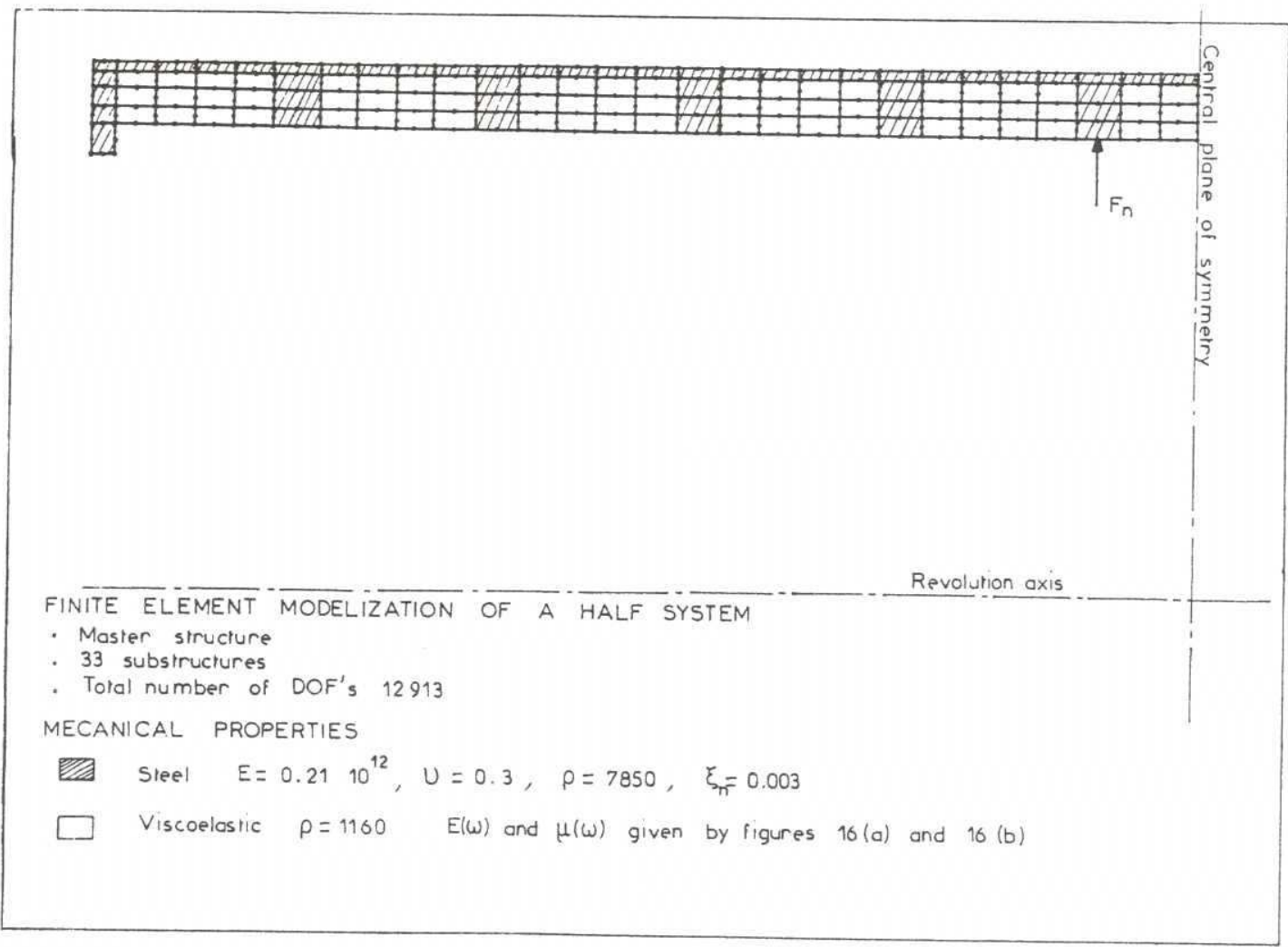

Fig. 15. Elastic structure with viscoelastic material. Modelization and mechanical properties. 


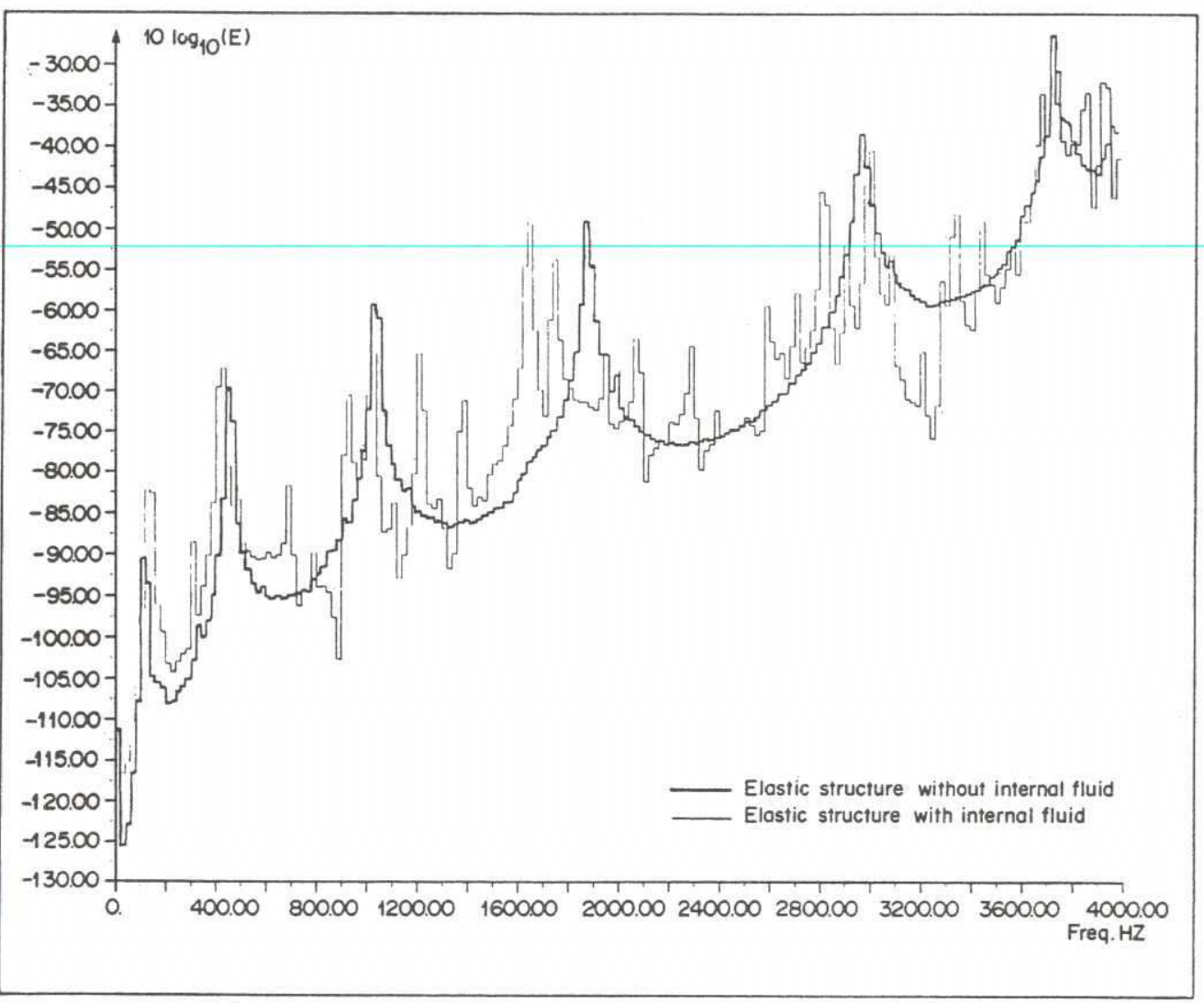

(a)

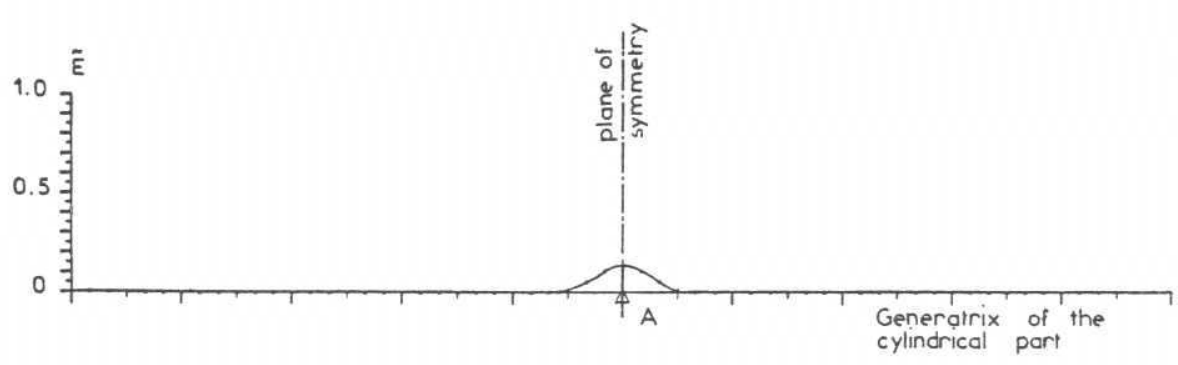

(b) with or without internal fluid

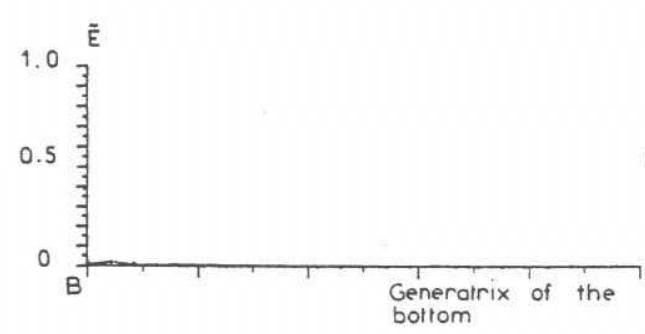

(c) without internal fluid

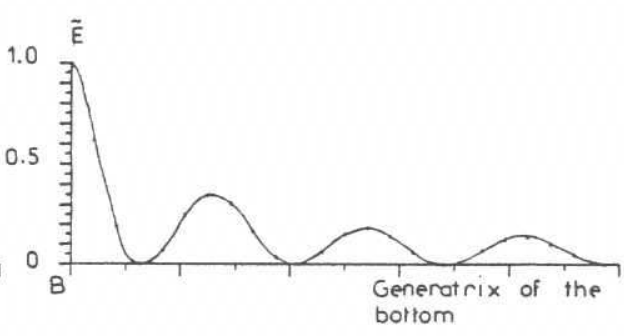

(d) with internal fluid

Fig. 14. Influence of the internal fluid on the vibrations of a closed elastic cylinder. (a) Frequency response function expressed in terms of energy. $E=$ energy of the normal acceleration integrated by frequency band of $20 \mathrm{~Hz}$ at observation point $B$. (b), (c), and (d) Spatial propagation of the normalized energy of the radial and normal acceleration $E$ in the band [1600-1700] $\mathrm{Hz}$. 

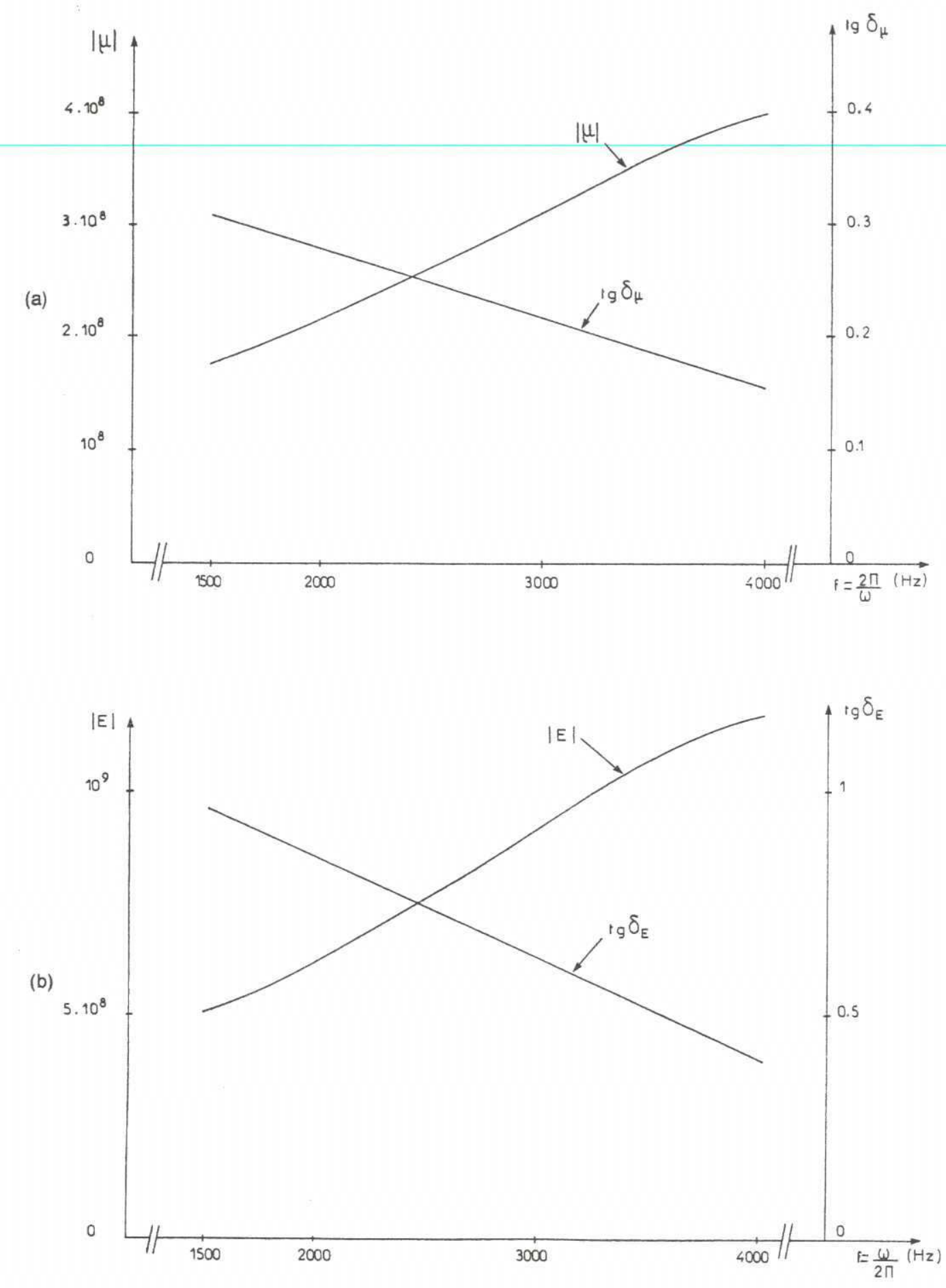

Fig. 16. (a) Complex Young's modulus. (b) Complex shear modulus. 


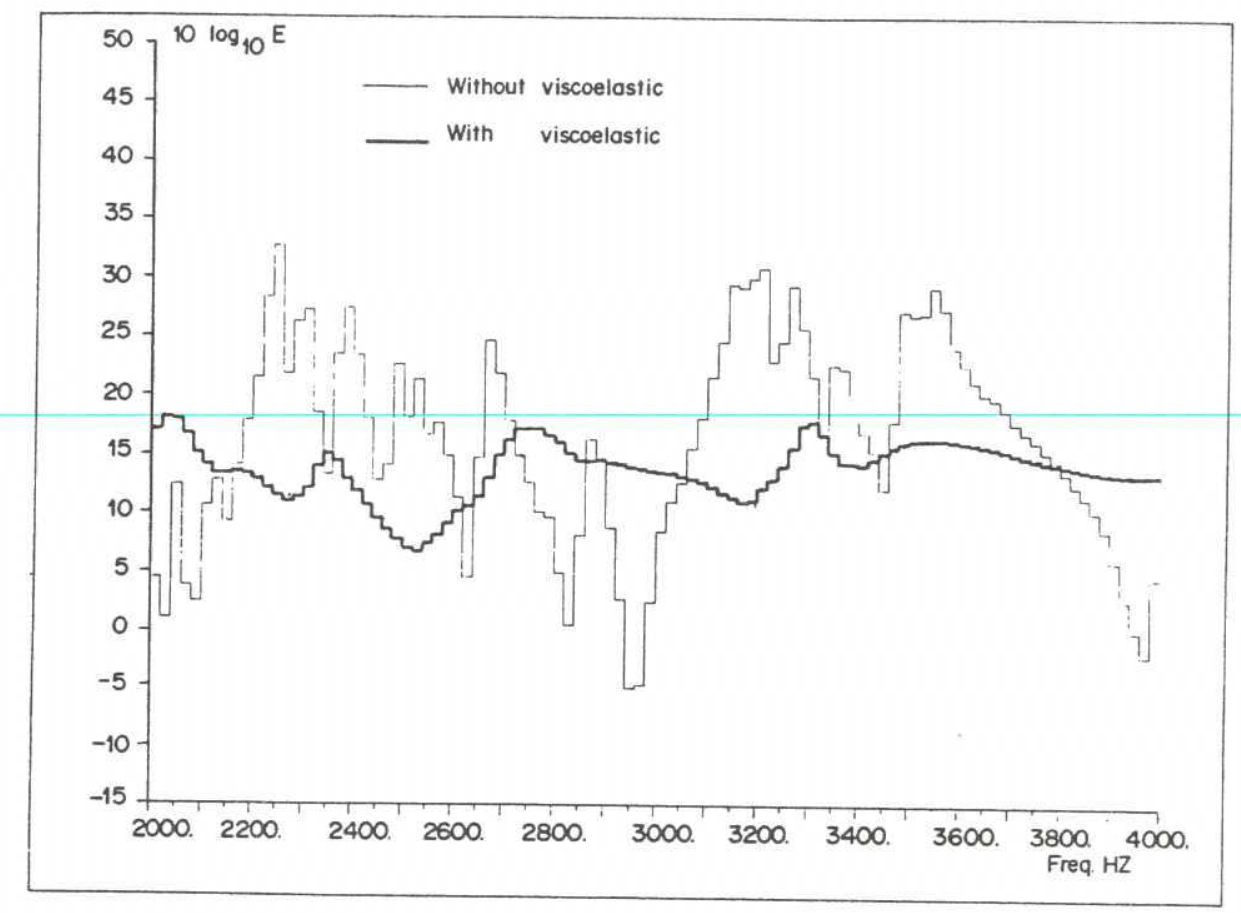

Fig. 17. Elastic structure with and without viscoelastic material. Frequency response function expressed in terms of energy. $E=$ energy of the radial acceleration integrated by frequency band of 20 $\mathrm{Hz}$ at the excitation point.
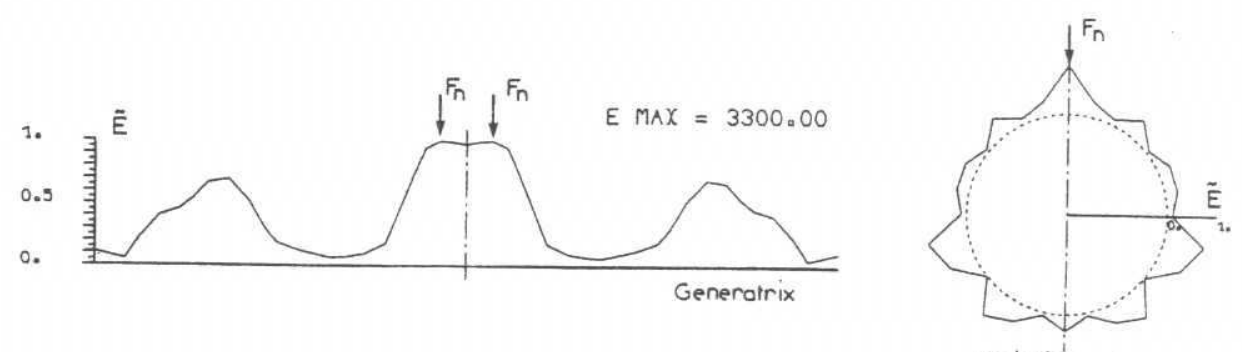

Excitation crown

WITHOUT VISCOELASTIC MATERIAL

(a)
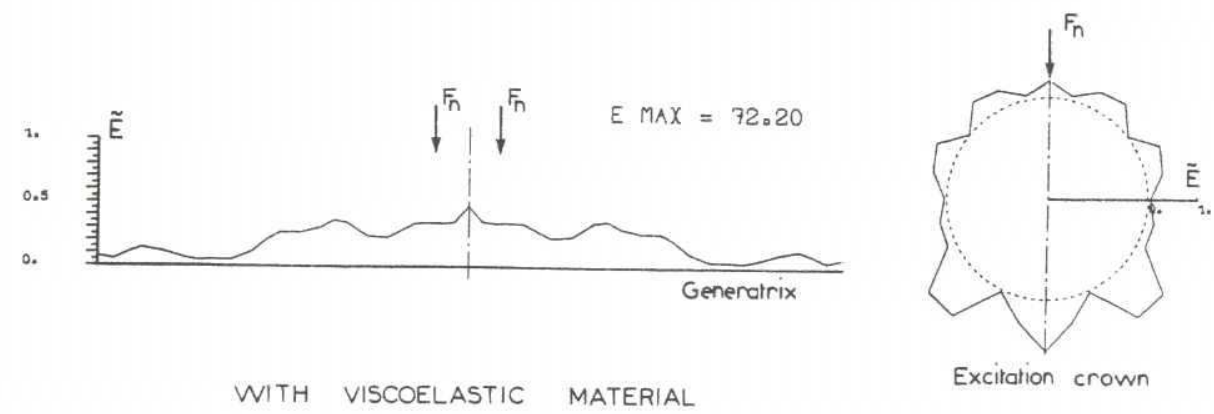

Fig. 18. Elastic structure with and without viscoelastic material. Spatial propagation of the normalized energy of the radial acceleration in a $B_{n}$ band. (a) Frequency band $B_{n}=[2200-2300] \mathrm{Hz}$. (b) Frequency band $B_{n}=[3100-3200] \mathrm{Hz}$. (Continued on following page.) 

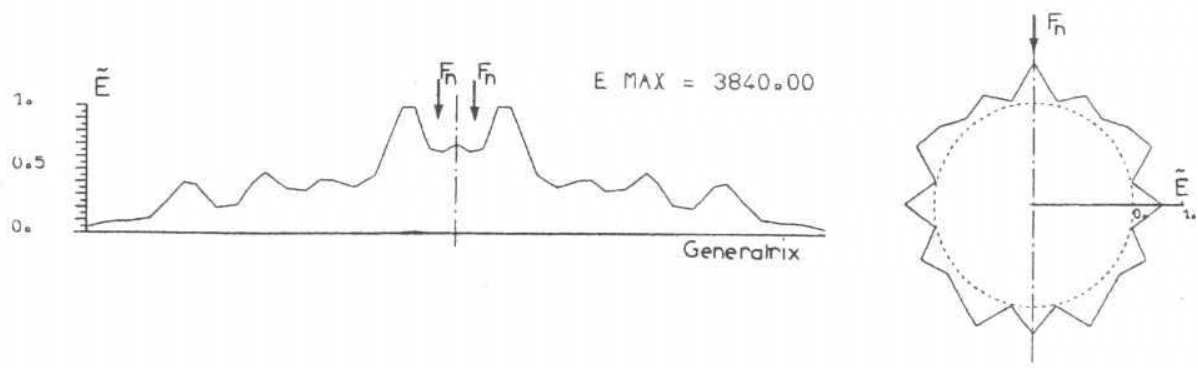

WITHOUT VISCOELASTIC MATERIAL

(b)
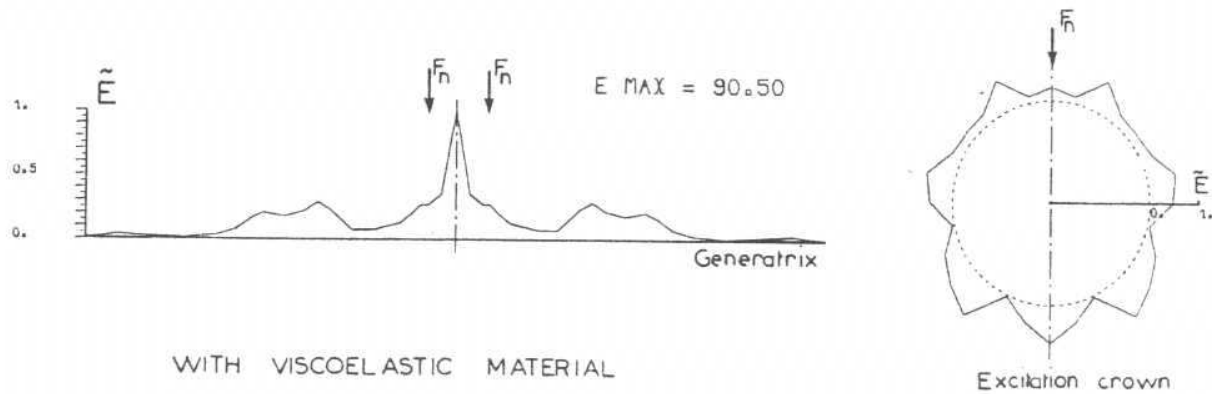

Fig. 18. continued.

\section{CONCLUSIONS}

The difficulties encountered in studying the dynamics of linear elastic media in the medium frequency range with modal techniques led us to develop a method supported by numerical processes. This approach can be used to address problems of fluid-structure interaction in the same frequency ranges, and in particular linear elasto-acoustical problems. We are currently attracted to this method so as to be able to process practical cases entailing modelization with a large number of degrees of freedom. The numerical analysis that we have carried out shows that this method can be used without difficulty in the main existing structural computation codes.

\section{REFERENCES}

1. J. D. Achenbach, Wave Propagation in Elastic Solids. North-Holland, Amsterdam (1980).

2. J. J. Angelini and P. M. Hutin, Exterior Neumann problem for Helmholtz equation. Problem of irregular frequencies. J. Recherche Aerospat. 3 (1983).

3. K. J. Bathe, ADINA-A finite element program for automatic dynamic incremental nonlinear analysis. Report 82448-1, Acoustics and Vibration Laboratory, Department of Mechanical Engineering, MIT, Cambridge, MA (1975).

4. K. J. Bathe, Finite Element Procedures in Engineering Analysis. Prentice-Hall, Englewood Cliffs, NJ (1982).

5. K. J. Bathe and E. L. Wilson, Numerical Methods in Finite Element Analysis. Prentice-Hall, Englewood Cliffs, NJ (1976).
6. J. T. Beale, Scattering frequencies of resonators. Commun. Pure App. Math. 36, 549-563 (1973).

7. T. Belytschko, H. J. Yen and R. Mullen, Mixed methods for time integration. J. Comput. Methods Appl. Mech. Engng 17/18, 259-275 (1979).

8. J. S. Bendat and A. G. Piersol, Random Data: An alysis and Measurement Procedures. Wiley Interscience, New York (1971).

9. A. Bensoussan, J. L. Lions and G. Papanicolaou, Asymptotic Analysis for Periodic Structure. NorthHolland, Amsterdam (1978).

10. S. Bergman and M. Schiffer, Kernel Functions and Elliptic Differential Equations in Mathematical Physics. Academic, New York (1953).

11. D. R. Bland, The Theory of Linear Viscoelasticity. Pergamon, London (1960).

12. F. Chabas and A. Desanti, Study and modelization of linear viscoelastic materials in the medium frequency range (in French). Report 403454RY442RONERA, France (1984).

13. Chowdhury, The truncated Lanczos algorithm for partial solution of the symmetric eigenproblems. Comput. Struct. 6, 439-446 (1976).

14. P. Ciarlet, Numerical analysis of the finite element method. Presses de l'université de Montreal, Canada (1976).

15. B. L. Clarkson, The derivation of modal densities from point impedances. J. Sound Vibr. 77, 583-584 (1981).

16. R. W. Clough and J. Penzien, Dynamic of Structures. McGraw-Hill, New York (1975).

17. D. M. Cole, D. D. Kosloff and J. B. Minster, A numerical boundary integral equation method for elastodynamics I. Bull. Seismol. Soc. Am. 68, 1331-1357 (1978).

18. L. Collatz, The Numerical Treatment of Differential Equations. Springer, New York (1966).

19. R. D. Cook, Concepts and Applications of Finite Element Analysis. Wiley, New York (1974). 
20. G. Coupry and C. Soize, Hydroelasticity and the field radiated by a slender elastic body into an unbounded fluid. J. Sound Vibr. 96, 261-273 (1984).

21. R. R. Craig and C. C. Chang, On the use of attachment modes in substructure coupling for dynamic analysis. Paper 77405, AIAA-ASME, 18th Struc. Dyn and Mat. Conf., San Diego (1977).

22. H. Cramer and M. R. Leadbetter, Stationary and Related Stochastic Processes. Wiley, New York (1967).

23. T. A. Cruse and F. J. Rizzo, A direct formulation and numerical solution of the general transient elastodynamic problems. Part I and Part II. J. Math. Anal. Appl. 22, 244-259, 341-355 (1968).

24. J. M. David, 3D hydro-elasto-acoustic of a stiffened cylindrical shell in low frequency range (in French). Report 363454RY080R- ONERA, France (1984).

25. J. M. David, F. Chabas and C. Soize, Study of the spatial propagation of vibratory energy in a heterogeneous structure in medium frequency range (in French). Report 413454RY452R- ONERA, France (1985).

26. J. M. David, A. Desanti and P. M. Hutin, Hydroelasto-acoustic calculations of a stiffened cylindrical shell in medium frequency range and experimental comparisons (in French). Report 433454RY454RONERA, France (1985).

27. A Desanti and C. Soize, Fluid-structure interaction in bounded domain in the medium frequency range (in french). Report 42894RN042R- ONERA, France (1984).

28. G. P. Destefano, Causes of instabilities in numerical integration techniques. Int. J. Comp. Math. 2, 123142 (1968)

29. J. L. Doob, Stochastic Processes. Wiley, New York (1967).

30. N. Dunford and J. T. Schwartz, Linear Operators $I$. Interscience, New York (1967)

31. C. A. Fellippa, Finite element and finite difference technique for the numerical solution of partial differential equations. Proceedings Conference on Computer Simulation, Montreal, Canada, July, 1973

32. S. J. Fenves, N. Perrone, A. R. Robinson and Schnobrich, Numerical and Computer Methods in Structural Mechanics. Academic, New York (1973).

33. G. E. Forsythe and W. R. Wason, Finite Difference Methods for Partial Differential Equations. Wiley, New York (1960).

34. L. E. Franks, Signal Theory. Prentice-Hall, Englewood Cliffs, NJ (1969).

35. D. Frederick and T. S. Chang, Continuum Mechanics. Allyn and Bacon, Boston (1965).

36. H. Freeman, Discrete Time Systems. Wiley, New York (1965).

37. A. Friedman, Stochastic Differential Equations and Applications, Vols. 1 and 2. Acdemic, New York (1975).

38. C. E. Froberg, Introduction to Numerical Analysis. Addison-Wesley, Reading, Mass. (1969).

39. Y. C. Fung, Foundations of Solids Mechanics. Prentice-Hall, Englewood Cliffs, NJ (1965).

40. Y. C. Fung, A First Course in Continuum Mechanics. Prentice-Hall, Englewood Cliffs, NJ (1969).

41. R. H. Gallagher, Finite Element Analysis Fundamentals. Prentice-Hall, Englewood Cliffs, NJ (1975).

42. P. Germain, Mécanique des Milieux Continus. Masson, Paris (1973).

43. R. I. Goldman, Vibration analysis by dynamic partitioning. AIAA J. 7 (1969)

44. L. Guikhman and A. V. Skorokhod, The Theory of Stochastic Processes. Springer, Berlin (1979).

45. P. Henrici, Error Propagation for Difference Methods. Wiley, New York (1963).
46. H. M. Hilber, T. J. R. Hughes and R. L. Taylor, Improved numerical dissipation for time integration algorithms in structural mechanics. Int. J. Earthquake Engng Struct. Dyn. 5, 283-292 (1977).

47. A. S. Householder, Principles of Numerical Analysis. McGraw-Hill, New York (1963).

48. T. J. R. Hughes and W. K. Liu, Implicit-explicit finite elements in transient analysis: stability theory. J. Appl. Mech. 45 (1978)

49. P. M. Hutin and J. J. Angelini, Correlation of the radiated noise by an immersed shell with its vibratory state (in french). Report 23454RV003R- ONERA, France (1980)

50. W. C. Hurty, J. D. Collins and G. C. Hart, Dynamic analysis of large structures by modal synthesis techniques. Comput. Struct. 1, 535-563 (1971).

51. W. C. Hurty and M. F. Rubinstein, Dynamics of Structures. Prentice-Hall, Englewood Cliffs, NJ (1964).

52. N. Ikeda and S. Watanabe, Stochastic Differential Equations and Diffusion Processes. North-Holland, Amsterdam (1981).

53. J. F. Imbert, Analyse des structures par éléments finis. Ecole nationale supérieure del'aeronautique et de l'espace, SUPAERO, CEPAD, Paris (1979).

54. E. Issaacson and H. B. Keller, Analysis of Numerical Methods. Wiley, New York (1966).

55. G. M. Jenkins and D. G. Watt, Spectral Analy sis and Its Applications. Holden Day, San Francisco (1968).

56. P. S. Jensen, The solution of large symmetric eigenproblems by sectioning. SIAM J. Numer. Anal. 9 (1972).

57. A. Jouan, A. Morvan and J. P. Allardot, Hydroelasto-acoustic measures at the Lac de Castillon (in french). Report 183454RY040R- ONERA, France (1982).

58. A. Jouan, A. Morvan and L. Guillaumie, Hydroelasto-acoustic measures in low and medium frequency ranges at the Lac de Castillon (in French). Report 373454RY082447R- ONERA, France (1984).

59. T. Kato, Perturbation Theory for Linear Operators. Springer, New York (1966).

60. P. Kree and C. Soize, Random Mechanics. Reidel, Dordrecht (1986). In French: Mecanique Aleatoire. Dunod, Paris (1983).

61. R. D. Krieg, Unconditional stability in numerical time integration method. Trans. ASME J. Appl. Mech. 417-421 (1973).

62. C. Lanczos, Applied Analysis. Prentice-Hall, Englewood Cliffs, NJ (1956).

63. A. W. Leissa, Vibration of shells. NASA SP-288, Washington, D.C. (1973)

64. S. Levy and J. P. D. Wilkinson, The Component Ele ment Method in Dynamics. McGraw-Hill, New York (1976).

65. Y. K. Lin, Probabilistic Theory of Structural Dynamics. McGraw-Hill, New York (1968).

66. R. H. Lyon, Statistical Energy Analysis of Dynamical Systems: Theory and Applications. MIT, Cambridge (1975).

67. S. G. Mikhlin, Mathematical Physics and Advanced Course. North-Holland, Amsterdam (1970).

68. D. P. Mondkar and G. H. Powell, Large capacity equation solver for structural analysis. Comput. Struct. 4, 699-728 (1974).

69. B. Nicolas-Vullierme, Harmonic vibrations of immersed structure. An incompressible approach (in french). Report 243454RY052R- ONERA, France (1983).

70. R. E. Nickell, Direct integration in structural dynamics. ASCE J. Engng Mech. Div. 99, 303-317 (1973).

71. B. Noble, Applied Linear Algebra. Prentice-Hall, Englewood Cliffs, NJ (1969). 
72. J. T. Oden and J. N. Reddy, An Introduction to the Mathematical Theory of Finite Elements. Wiley, New York (1976).

73. R. Ohayon and R. Valid, True symmetric variational formulations for fluid-structure interaction in bounded domains-finite elements results, in $\mathrm{Nu}$ merical Methods in Coupled Systems (Edited by R. W. Lewis, P. Bettess, and E. Hinton). Wiley, New York (1984)

74. A. Oppenheim and R. Schafer, Digital Signal Processing. Prentice-Hall, Englewood Cliffs, NJ (1974).

75. A. Papoulis, Signal Analysis. McGraw-Hill, New York (1977).

76. G. Piazzoli and R. Ohayon, Acoustic field radiated by elastic submarines (in French). Report 263454RY050-052R- ONERA, France (1983).

77. W. Prager, Introduction to Mechanics of Continua. Ginn and Co., New York (1961).

78. J. S. Przemieniecki, Theory of Matrix Structural Analysis. McGraw-Hill, New York (1968)

79. L. R. Rabiner and B. Gold, Theory and Application of Digital Signal Processing. Prentice-Hall, Englewood Cliffs, NJ (1975).

80. M. Roseau, Asymptotic Wave Theory. North-Holland, Amsterdam (1976).

81. Y. A. Rozanov, Stationary Random Processes. Holden Day, San Francisco (1967).

82. M. F. Rubinstein, Structural Systems. Statics, Dynamics, and Stability. Prentice-Hall, Englewood Cliffs, NJ (1970).

83. M. F. Rubinstein and D. E. Wikholm, Analysis group iteration using substructures. ASCE J. Struct. Div. 94 (1968).

84. E. Sanchez-Palencia, Nonhomogeneous Media and Vibration Theory. Springer, Berlin (1980).

85. C. Soize, The Mathematics of Deterministic and Random Signal Theory. ENSTA, Paris (1983).

86. C. Soize, Hydroelasticity of axisymmetric slender bodies into a compressible unbounded fluid (in french). Report 173454RY032R- ONERA, France (1982).

87. C. Soize, Medium frequency linear vibrations of anisotropic elastic structures. J. Recherche Aerospat. ONERA France $\mathbf{5}$ (1982).

88. C. Soize and F. Chabas, Hydroelasticity of a slender elastic body into an unbounded fluid in the medium frequency range (in French). Report 423454RY441R ONERA, France (1985).

89. C. Soize, J. M. David, A. Desanti and P. M. Hutin,
Development of a method to analyze the fluid-structure interactions in the medium frequency range (in French). Report 283454RY071R- ONERA, France (1983).

90. C. Soize, J. M. David, A. Desanti and P. M. Hutin, Acostic field radiated by shells in the medium frequency range. Extension of ADINA (in French). Report 273454RY063R- ONERA, France (1983).

91. C. Soize, J. M. David, A. Desanti and P. M. Hutin, Medium frequency vibrations of a 3D stiffened cylindrical shell in vacuo and in immersion, in several configurations, and experimental comparisons. Vols. I and II (in french). Report 343454RY081R-ONERA, France (1984).

92. T. T. Soong, Random Differential Equations in Science and Engineering. Academic, New York (1973).

93. G. Strang and G. J. Fix, An Analysis of the Finite Element Method. Prentice-Hall, Englewood Cliffs, NJ (1973).

94. S. Timoshenko and J. N. Goodier, Theory of Elasticity. McGraw-Hill, New York (1951).

95. K. N. Tong, Theory of Mechanical Vibration. Wiley, New York (1960).

96. C. Truesdell, The Elements of Continuum Mechanics. Springer, Berlin (1966).

97. R. Valid, Mechanics of Continuous Media and Analysis of Structures. North-Holland, Amsterdam (1981).

98. R. S. Varga, Matrix Iterative Analysis. PrenticeHall, Englewood Cliffs, NJ (1962).

99. K. Wahizu, Variational Methods in Elasticity and Plasticity. Pergamon, Elmsford, NY (1967).

100. The Mathematics of Finite Elements and Applications (Edited by J. R. Whiteman). Academic, London (1973).

101. J. H. Wilkinson, Rounding Errors in Algebraic Processes. Prentice-Hall, Englewood Cliffs, NJ (1962).

102. E. L. Wilson, Structural analysis of axisymmetric solids. AIAA J. 3, No 12, Dec. (1965).

103. E. L. Wilson, K. J. Bathe and W. P. Doherty, Direct solution of large systems of linear equations. Comput. Struct. 4, 363-372 (1974).

104. D. M. Young, Iterative Solution of Large Linear Sys tems. Academic, New York (1971).

105. O. C. Zienkiewicz, The Finite Element Method in Engineering Science. McGraw-Hill, New York (1971).

106. K. N. Tong, Theory of Mechanical Vibration. Wiley, New York (1960). 